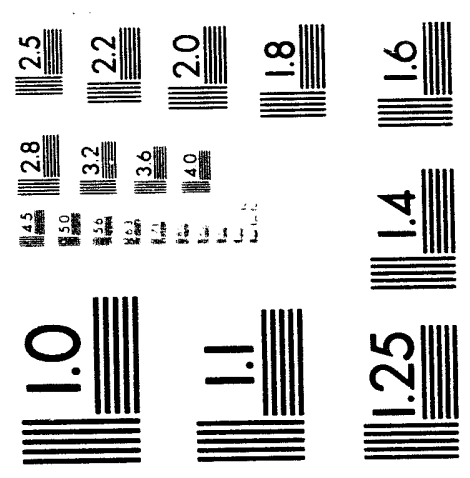



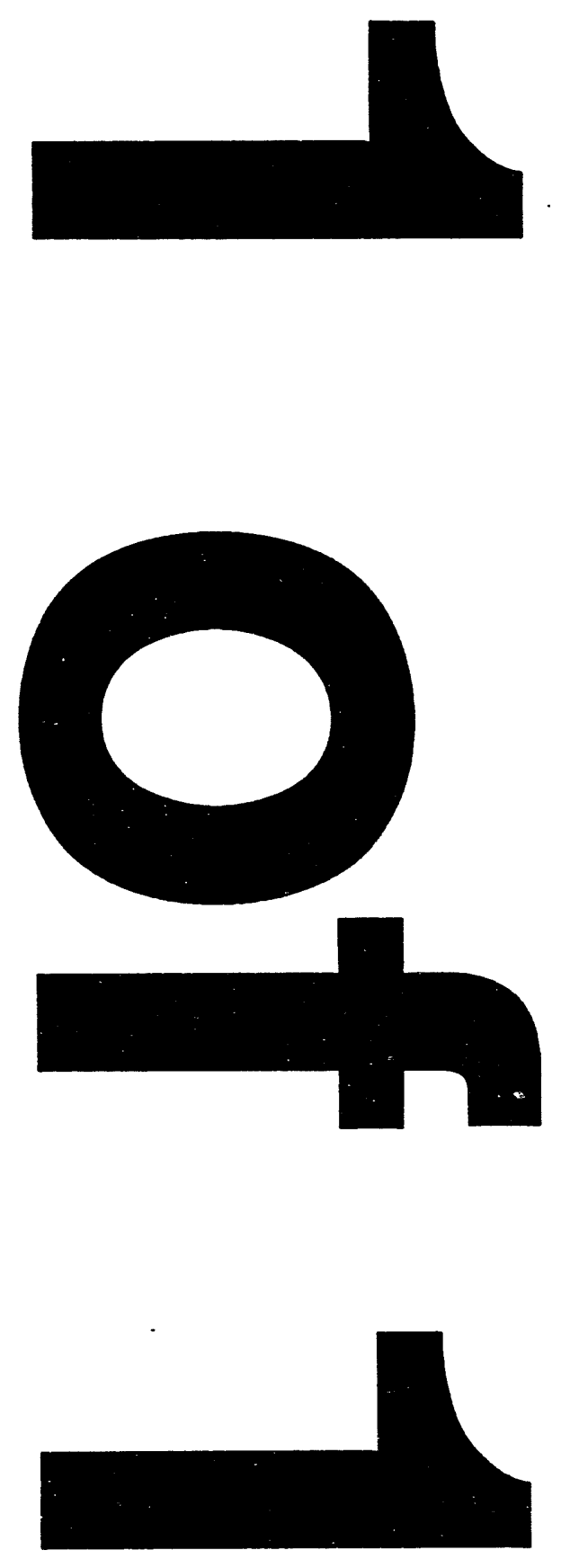


\title{
EMpIRICAL DistribUtions Of RADIONUCLIDES, FROM RWMIS DATA
}

\author{
Corwin L. Atwood and Melinda J. Schlafman
}

Apri1 1993

Idaho National Engineering Laboratory

EG\&G Idaho, Inc.

Idaho Fal1s, Idaho 83415

Prepared for the

U.S. Department of Energy

Office of Environmental Restoration and Waste Management Under DOE Field Office, Idaho

Contract DE-ACO7-76ID01570

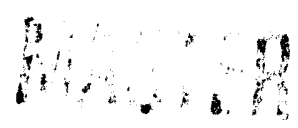




\section{ABSTRACT}

The RWMIS data base gives data on each shipment of waste received at the Transuranic Storage Area, including the total volume of the shipment and the activity ( $\mathrm{C} i$ ) of each nuclide in the shipment. This report assumes that the RWMIS numbers are correct, and considers the waste containers now retrievably stored at the Transuranic Storage Area. The total decay-corrected activities are summarized for several classes, such as for transuranic (TRU) waste and non-TRU waste, for $\alpha$-emitters and $\beta / \gamma$-emitters, by waste originator and by current storage location. The total activity for each nuclide is also given.

The empirical distributions are then given for a number of classes and individual nuclides, reflecting the variability between waste shipments. They are expressed in terms of $\mathrm{mCi} / \mathrm{cu}-\mathrm{ft}$; for fissionable nuclides, the same information is also expressed in terms of $\mathrm{mg} / \mathrm{cu}-\mathrm{ft}$; finally, the distribution is also given for the committed effective dose equivalent from inhalation, expressed in Mrem/cu-ft. The empirical distributions can be used for simulating the contents of a random waste container with a postulated volume. Examples are given illustrating the uses and limitations of the results. 


\section{ACKNOWLEDGMENTS}

Cathy Barnard provided useful information about the data bases. Larry $G$. Blackwood contributed helpful ideas and insights as the mathematical analysis method was being developed. The reviews by Steve Bengston, Ralph Falconer, and John Poloski were helpful. 


\section{CONTENTS}

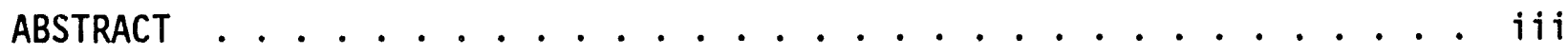

ACKNOWLEDGMENTS .................... iv

DESCRIPTION OF THE DATA SETS ................. 1

ANALYSIS METHOD ...................... 2

RESULTS ..................... 11

Tabulated Empirical Distributions . . . . . . . . . . . 11

How to Read the Tables ................... 13

How to Use the Tables for Simulations . . . . . . . . . . 27

Cautions ....................... 30

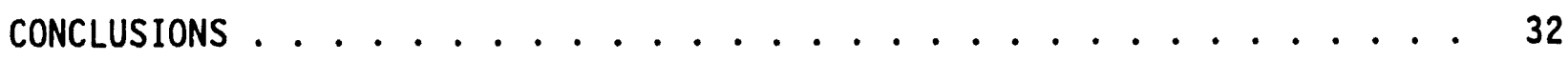

APPENDIX A: DETAILS OF PROGRAMMING ............. 33

dBASE Calculations, Including Decayed Activity ..... 33

Fortran Programs to Summarize Data and Get Normalized Activities . 45

SAS Programs to Obtain Empirical Distributions ......... 65

References ................... 68

APPENDIX B: ANALYSIS METHODS TRIED . . . . . . . . . . . 71

First Techniques Tried ................ . . 71

Problems with the First Techriques . . . . . . . . . . 72

Next Techniques Tried, and Ultimately Used .......... 73

\section{TABLES}

Table 1. Retrievably stored TSA nuclides in RWMIS. . . . . . . . . . 3

Table 2. Tally of shipments, volume, and decayed activity. . . . . . 5 5

Table 3. Tally of containers of different types and volumes. . . . . . 8 8

Table 4. Distributions of major classes of nuclides, mCi/cu-ft. . . . 14

Table 5. Distributions of individual nuclides, $\mathrm{mCi} / \mathrm{cu}-\mathrm{ft} . \ldots 15$

Table 6. Distributions of activity from major generators, $\mathrm{mCi} / \mathrm{cu}-\mathrm{ft}$. . 18

Table 7. Distributions of activity by present storage area, $\mathrm{mCi} / \mathrm{cu}-\mathrm{ft}$. . 20

Table 8. Distributions of fissionable nuclides by class, $\mathrm{mg} / \mathrm{cu}-\mathrm{ft} . . .21$

Table 9. Distributions of individual fissionable nuclides, $\mathrm{mg} / \mathrm{cu}-\mathrm{ft}$. . . 22

Table 10. Exact distributions for classes occurring in few shipments. . 23

Table 11. Overall committed effective dose equivalent from inhalation. . 25

Table 12. Histogram of distribution for TRU waste. . . . . . . 28

Table 13. Histograms for ILTSF non-TRU waste and inhalation dose. . . 30 


\section{EMPIRICAL DISTRIBUTIONS OF RAdionuclide Activities, FROM RWMIS DATA}

\section{DESCRIPTION OF THE DATA SETS}

RWMIS contains three subsets: the BAD, the Historical, and the Current. The "BAD" Data (an acronym for Best Available Data) contains data for shipments before 1971. Al1 the waste containers in this data base are now buried, and therefore are not considered in this report. The "Historical Data" corresponds to shipments from 0ct. 22, 1970 through Dec. 1, 1983; some of these containers are now retrievably stored. The "Current Data" corresponds to shipments from Dec. 1, 1983 on, all of which are retrievably stored.

For the present task, Deborah Litteer, custodian of RWMIS, downloaded the Current Data, and that portion of the Historical Data corresponding to aboveground containers, from the NOMAD system used by RWMIS to dBASE files. The effective date of the download was Dec. 23, 1992. The number of records agreed in the original and downloaded files, and the total activities of selected nuclides also agreed to several significant digits. This downloading apparently produced some (negligible) inaccuracy: typically, activities are entered in RWMIS to only 3 or 4 significant digits, but the dBASE files normally appeared to have many significant digits. Considering the fact that the original activities are only rough estimates, the inaccuracy introduced by the download is negligible.

The accuracy of the RWMIS data is not addressed here. This analys is is not an endorsement of the RWMIS data's accuracy. Based on past experience with RWMIS, we knew that when the container type code is $B L X$, the reported container volume should be divided by 10; we would have done this if that code had occurred. We also have identified one case for which it appears that two shipments were given one identifier; because we could not tell which containers 
went with which shipment, we wrote our software to combine the shipments in any such case, adding the activities of each nuclide and summing the container volumes. We are not aware of other anomalies in the RWMIS data. As a guiding principle, however, one must realize that there are approximately 20,000 shipments, and that the originally entered nuclide activities for these shipments were probably not all obtained from direct measurements of the shipments.

\section{ANALYSIS METHOD}

Software was written in the dBASE language to decay the radionuclides to $2 / 17 / 93$, and to provide a summary of the activities of the individual nuclides. This summary is shown in Table 1. Only four significant digits are shown for each activity. Because of the generation of daughters, some nuclides appear in the present inventory which were not reported in the original inventories. This is one more sign that the original inventories should not be taken as more than an approximation. Another consequence of the generation of daughters is that some activities have increased over time; for example, Am-241, a daughter of Pu241, has increased from what was originally reported. No daughter nucl ide was generated if its activity would be less than $2 \mathrm{Ci}$. Therefore, some of the small listed activities are incorrect, because they do not include the activity $(<2$ Ci) of newly generated atoms. Details of the decay calculations are given in Appendix A.

The data base was scanned and tallied, to show the shipments' number and total volume, for the various nuclides, present storage locations, and waste generators. This tally is shown in Table 2. For example, from Table 2 there was one shipment containing any Ac-227. This shipment had total volume of $7.35 \mathrm{cu}-\mathrm{ft}$ (which equals 55 gal), and the total decayed activity of the Ac-227 is $0.04079 \mathrm{Ci}$. By contrast, there were 18863 shipments containing Am-241. The numbers of shipments for the individual nuclides add up to more than the total number of shipments, because most shipments contain more than one nuclide, and similarly the shipment volumes for the nuclides add up to more than the total volume. The activities for the individual nuclides, on the other hand, add up to the total activity. Because every shipment came from one waste generator and 
Table 1. Retrievably stored TSA nuclides in RWMIS. ${ }^{a}$

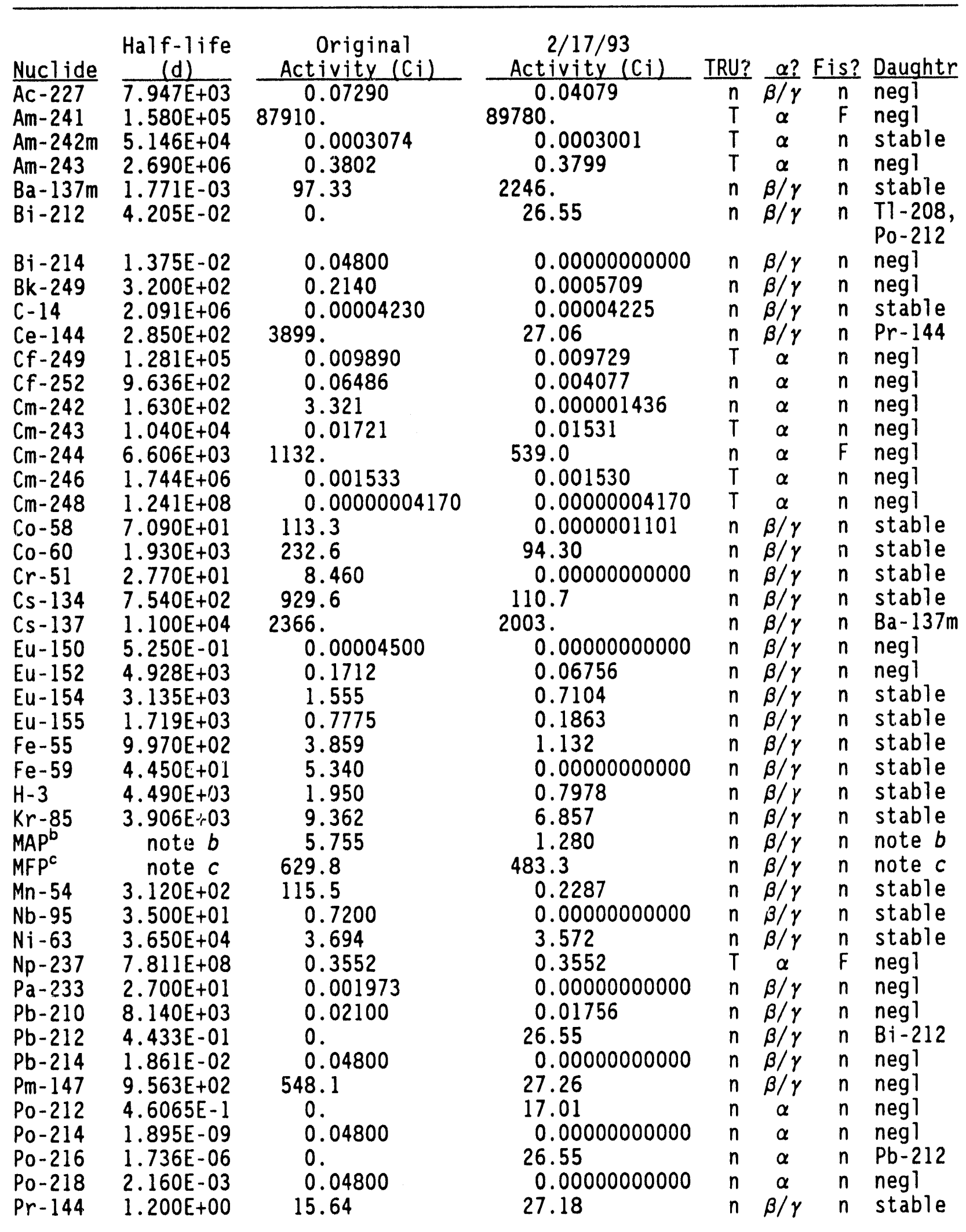


Table 1. (Cont.)

\begin{tabular}{|c|c|c|c|c|c|c|c|}
\hline & $\begin{array}{l}1.040 E+03 \\
3.201 E+04 \\
8.796 E+06 \\
2.394 E+06 \\
5.256 E+03 \\
1.369 E+08 \\
3.660 E+00 \\
5.840 E+05 \\
2.102 E+03 \\
3.461 E-04 \\
6.435 E-04 \\
3.820 E+00 \\
3.723 E+02 \\
1.007 E+03 \\
1.062 E+04 \\
1.140 E+02 \\
7.774 E+07 \\
6.982 E+02 \\
2.752 E+07 \\
5.110 E+12 \\
2.410 E+01 \\
2.120 E-03 \\
6.319 E-03 \\
2.555 E+04 \\
5.840 E+07 \\
8.979 E+07 \\
2.570 E+11 \\
8.541 E+09 \\
1.632 E+12 \\
n 0 t e d \\
2.670 E+00 \\
2.440 E+02 \\
6.400 E+01\end{array}$ & $\begin{array}{c}0.05764 \\
67590 . \\
40160 . \\
9837 . \\
281600 . \\
0.9451 \\
0 . \\
0.04802 \\
0.2750 \\
1.300 \\
0 . \\
0.04800 \\
106.2 \\
6.087 \\
2116 . \\
0.7350 \\
0.001760 \\
0 . \\
0.01998 \\
0.3301 \\
0.00009280 \\
0 . \\
0.0001070 \\
30.02 \\
898.6 \\
3.359 \\
0.06669 \\
0.001199 \\
0.1171 \\
14.89 \\
101.8 \\
0.001100 \\
67.23\end{array}$ & $\begin{array}{c}0.01479 \\
60800 . \\
40150 . \\
9825 . \\
161100 . \\
0.9451 \\
26.55 \\
0.04790 \\
0.03062 \\
0.00000000000 \\
26.55 \\
0.00000000000 \\
0.1865 \\
1.647 \\
1778 . \\
0.00001548 \\
0.001760 \\
26.55 \\
0.01998 \\
0.3301 \\
0.00000000000 \\
9.540 \\
0.00000000000 \\
26.04 \\
898.5 \\
5.778 \\
0.06669 \\
0.001199 \\
0.1171 \\
12.44 \\
2019 . \\
0.00000001868 \\
0.00000000234\end{array}$ & $\begin{array}{l}n \\
T \\
T \\
T \\
n \\
T \\
n \\
n \\
n \\
n \\
n \\
n \\
n \\
n \\
n \\
n \\
n \\
n \\
n \\
n \\
n \\
n \\
n \\
n \\
n \\
n \\
n \\
n \\
n \\
n \\
n \\
n \\
n\end{array}$ & $\begin{array}{c}\alpha \\
\alpha \\
\alpha \\
\alpha \\
\beta / \gamma \\
\alpha \\
\alpha \\
\alpha \\
\beta / \gamma \\
\beta / \gamma \\
\alpha \\
\alpha \\
\beta / \gamma \\
\beta / \gamma \\
\beta / \gamma \\
\beta / \gamma \\
\beta / \gamma \\
\alpha \\
\alpha \\
\alpha \\
\beta / \gamma \\
\beta / \gamma \\
\alpha \\
\alpha \\
\alpha \\
\alpha \\
\alpha \\
\alpha \\
\alpha \\
\beta / \gamma \\
\beta / \gamma \\
\beta / \gamma \\
\beta / \gamma\end{array}$ & $\begin{array}{l}n \\
F \\
F \\
n \\
F \\
n \\
n \\
n \\
n \\
n \\
n \\
n \\
n \\
n \\
n \\
n \\
n \\
n \\
n \\
n \\
n \\
n \\
n \\
n \\
F \\
n \\
F \\
n \\
n \\
n \\
n \\
n \\
n\end{array}$ & $\begin{array}{l}\text { negl } \\
U-234 \\
\text { negl } \\
\text { negl } \\
\text { Am-241 } \\
\text { negl } \\
\text { Rn-220 } \\
\text { negl } \\
\text { negl } \\
\text { stable } \\
\text { Po-216 } \\
\text { neg1 } \\
\text { negl } \\
\text { negl } \\
\text { Y-90 } \\
\text { stable } \\
\text { stable } \\
\text { Ra-224 } \\
\text { negl } \\
\text { negl } \\
\text { negl } \\
\text { negl } \\
\text { negl } \\
\text { Th-228 } \\
\text { negl } \\
\text { negl } \\
\text { neg1 } \\
\text { negl } \\
\text { neg1 } \\
\text { note d } \\
\text { stable } \\
\text { stable } \\
\text { negl }\end{array}$ \\
\hline
\end{tabular}

a. The activities are rounded off to four significant digits, the maximum accuracy of any shipment's reported activity. They are printed in decimal rather than scientific notation to make the large activities more obvious to the eye. TRU nuclides are shown by a T, non-TRU nuclides by $n$. $\alpha$-emitters and $\beta / \gamma$ emitters are shown by $\alpha$ and $\beta / \gamma$, respectively. Fissionable nuclides are shown by $F$, non-fissionable nuclides by $n$. The daughters are shown as "negl" if less than $2 \mathrm{Ci}$ of the daughter would be generated. The daughters are shown as "stable" if they are not radioactive.

b. "Mixed Activation Products," treated as Co-60.

c. "Mixed Fission Products," treated as a mixture of half Cs-137 and half Sr-90.

d. "Unidentified $\beta / \gamma$-emitters," treated as Cs-137. 
Table 2. Tally of shipments, volume, and decayed activity.

\begin{tabular}{|c|c|c|c|}
\hline & Shipments & $\begin{array}{r}\text { Volume } \\
(\mathrm{cu}-\mathrm{ft})\end{array}$ & $\begin{array}{c}2 / 17 / 93 \\
\text { Activity (Ci) }\end{array}$ \\
\hline \multirow[t]{2}{*}{$\begin{array}{l}\text { Total } \\
\text { TRU } \\
\text { Non-TRU } \\
\alpha \text {-emitters } \\
\beta / \gamma \text {-emitters } \\
\text { Fissionable }\end{array}$} & $\begin{array}{l}19521 \\
19086 \\
19107 \\
19168 \\
18814 \\
19166\end{array}$ & $\begin{array}{l}2287042.84 \\
2268701.20 \\
2281803.53 \\
2283910.69 \\
2244506.53 \\
2283901.34\end{array}$ & $\begin{array}{l}372200 . \\
200600 . \\
171600 . \\
202200 . \\
170000 . \\
353300 .\end{array}$ \\
\hline & \multicolumn{3}{|c|}{ By Nuclide } \\
\hline$\frac{\text { Nuclide }}{A c-227}$ & & 7.35 & 0.04079 \\
\hline Am-241 & 18488 & 2193758.64 & 89780 . \\
\hline Am-242m & & 10.00 & 0.0003001 \\
\hline $\begin{array}{l}\text { Am-243 } \\
B a-137 m\end{array}$ & 22 & $\begin{array}{r}6196.17 \\
51287.09\end{array}$ & $\begin{array}{r}0.3799 \\
2246\end{array}$ \\
\hline $\begin{array}{l}\text { Ba-137m } \\
B i-212\end{array}$ & $\begin{array}{r}459 \\
54\end{array}$ & $\begin{array}{l}51287.99 \\
13940.21\end{array}$ & $\begin{array}{l}2246 . \\
26.55\end{array}$ \\
\hline $\begin{array}{l}B i-212 \\
B i-214\end{array}$ & $\begin{array}{r}54 \\
0\end{array}$ & $\begin{array}{r}1394.21 \\
0.00\end{array}$ & $\begin{array}{l}26.55 \\
0.00000000000\end{array}$ \\
\hline Bk-249 & 4 & 2280.00 & 0.0005709 \\
\hline$C-14$ & 2 & 1440.00 & 0.00004225 \\
\hline Ce-144 & 45 & 399.41 & 27.06 \\
\hline$C f-249$ & 4 & 1567.35 & 0.009729 \\
\hline Cf -252 & 9 & 4102.06 & 0.004077 \\
\hline $\mathrm{Cm}-242$ & 2 & 960.00 & 0.000001436 \\
\hline $\mathrm{Cm}-243$ & 2 & 10.00 & 0.01531 \\
\hline $\mathrm{Cm}-244$ & 15 & 4872.23 & 539.0 \\
\hline $\mathrm{Cm}-246$ & 3 & 1474.67 & 0.001530 \\
\hline $\mathrm{Cm}-248$ & 1 & 720.00 & 0.00000004170 \\
\hline Co-58 & 7 & 35.00 & 0.0000001101 \\
\hline Co-60 & 19 & 848.41 & 94.30 \\
\hline$C r-51$ & 0 & 0.00 & 0.00000000000 \\
\hline$C s-134$ & 34 & 327.00 & 110.7 \\
\hline$C s-137$ & 167 & 6134.85 & 2003. \\
\hline Eu-150 & 0 & 0.00 & 0.00000000000 \\
\hline$E u-152$ & 9 & 4574.35 & 0.06756 \\
\hline Eu-154 & 15 & 4038.00 & 0.7104 \\
\hline Eu-155 & 10 & 205.00 & 0.1863 \\
\hline $\mathrm{Fe}-55$ & 4 & 1450.00 & 1.132 \\
\hline $\mathrm{Fe}-59$ & 0 & 0.00 & 0.00000000000 \\
\hline $\mathrm{H}-3$ & 5 & 5362.67 & 0.7978 \\
\hline $\mathrm{Kr}-85$ & 2 & 10.00 & 6.857 \\
\hline $\begin{array}{l}\text { MAPD } \\
\text { MFPC }\end{array}$ & & 265.00 & 1.280 \\
\hline & & & \\
\hline $\begin{array}{l}M n-54 \\
N h-95\end{array}$ & $\begin{array}{r}26 \\
0\end{array}$ & 153.00 & 0.2287 \\
\hline $\mathrm{Nb}-95$ & 0 & 0.00 & 0.00000000000 \\
\hline $\mathrm{Ni}-63$ & 3 & 730.00 & 3.572 \\
\hline $\mathrm{Np}-237$ & 38 & 13026.83 & 0.3552 \\
\hline$P_{a}-233$ & 0 & 0.00 & 0.00000000000 \\
\hline
\end{tabular}


Table 2. (Cont.)

\begin{tabular}{|c|c|c|c|}
\hline 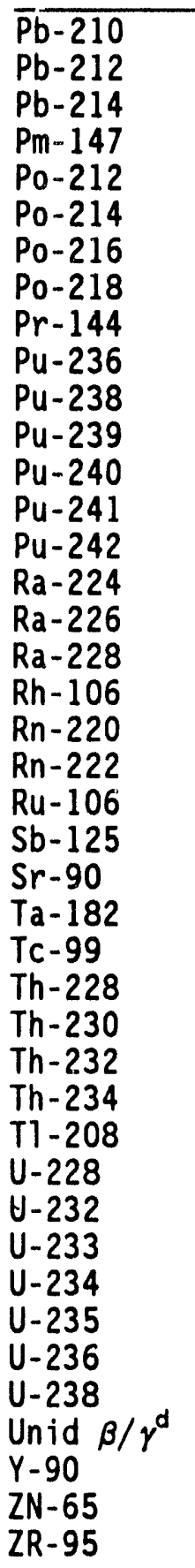 & $\begin{array}{r}1 \\
54 \\
0 \\
3 \\
54 \\
0 \\
54 \\
0 \\
45 \\
299 \\
18400 \\
19059 \\
18881 \\
18386 \\
18329 \\
54 \\
2 \\
1 \\
0 \\
54 \\
0 \\
13 \\
11 \\
32 \\
1 \\
3 \\
54 \\
1 \\
105 \\
0 \\
54 \\
0 \\
54 \\
152 \\
18410 \\
1107 \\
31 \\
331 \\
6 \\
334 \\
2 \\
8\end{array}$ & $\begin{array}{r}7.35 \\
13940.21 \\
0.00 \\
17.35 \\
13940.21 \\
0.00 \\
13940.21 \\
0.00 \\
399.41 \\
7540.70 \\
2217049.04 \\
2268443.85 \\
2213546.71 \\
2180718.05 \\
2181792.61 \\
13940.21 \\
14.35 \\
7.35 \\
0.00 \\
13940.21 \\
0.00 \\
250.00 \\
208.00 \\
315.00 \\
5.00 \\
847.35 \\
13940.21 \\
720.00 \\
25332.08 \\
0.00 \\
13940.21 \\
0.00 \\
13940.21 \\
56010.22 \\
2225539.37 \\
311137.15 \\
10070.01 \\
32485.69 \\
45.41 \\
47015.14 \\
1440.00 \\
57.41\end{array}$ & $\begin{array}{l}0.01756 \\
26.55 \\
0.00000000000 \\
27.26 \\
17.01 \\
0.00000000000 \\
26.55 \\
0.00000000000 \\
27.18 \\
0.01479 \\
60800 . \\
40150 . \\
9825 . \\
161100 . \\
0.9451 \\
26.55 \\
0.04790 \\
0.03062 \\
0.00000000000 \\
26.55 \\
0.00000000000 \\
0.1865 \\
1.647 \\
1778 . \\
0.00001548 \\
0.001760 \\
26.55 \\
0.01998 \\
0.3301 \\
0.00000000000 \\
9.540 \\
0.00000000000 \\
26.04 \\
898.5 \\
5.778 \\
0.06669 \\
0.001199 \\
0.1171 \\
12.44 \\
2019 . \\
0.00000001868 \\
0.00000000234\end{array}$ \\
\hline
\end{tabular}

By Location

Location $^{e}$

TSA

Total

TRU

Non-TRU

$\begin{array}{ll}2284317.04 & 363700 . \\ 2265975.40 & 200500 . \\ 2279125.73 & 163200 .\end{array}$


Table 2. (Cont.)

\begin{tabular}{|c|c|c|c|}
\hline $\begin{array}{l}\text { ILTSF } \\
\text { Total } \\
\text { TRU } \\
\text { Non-TRU }\end{array}$ & $\begin{array}{l}342 \\
342 \\
336\end{array}$ & $\begin{array}{l}2725.80 \\
2725.80 \\
2677.80\end{array}$ & $\begin{array}{c}8489 . \\
100.3 \\
8388 .\end{array}$ \\
\hline & \multicolumn{3}{|c|}{ By Waste Generator } \\
\hline $\begin{array}{l}\text { Generator } \\
\text { ALE } \\
\text { ANL } \\
\text { ARA } \\
\text { BCL } \\
\text { BEN } \\
\text { BET } \\
\text { CFA } \\
\text { CPP } \\
\text { D+D } \\
\text { LOF } \\
\text { MRC } \\
\text { NRF } \\
\text { RFO } \\
\text { TAN } \\
\text { TRA } \\
\text { WMC }\end{array}$ & $\begin{array}{r}407 \\
227 \\
4 \\
13 \\
1 \\
78 \\
2 \\
29 \\
1 \\
1 \\
446 \\
37 \\
17960 \\
5 \\
42 \\
268\end{array}$ & $\begin{array}{r}54076.28 \\
1531.35 \\
356.41 \\
7572.88 \\
7.35 \\
15301.31 \\
7.67 \\
1720.60 \\
7.35 \\
7.35 \\
137598.34 \\
246.16 \\
1913380.83 \\
31.41 \\
357.60 \\
154839.93\end{array}$ & $\begin{array}{c}6002 . \\
6812 . \\
11.94 \\
120.6 \\
18.48 \\
1586 . \\
0.04360 \\
1330 . \\
2.488 \\
1.189 \\
61000 . \\
428.5 \\
280500 . \\
16.00 \\
836.7 \\
13480 .\end{array}$ \\
\hline
\end{tabular}

a. The activities are rounded off to four significant digits, as in Table 1 .

b. "Mixed Activation Products," as in Table 1.

c. "Mixed Fission Products," as in Table 1.

d. "Unidentified $\beta / \gamma$-emitters," as in Table 1.

e. The locations given as text in the data base are the Transuranic Storage Area (TSA), and the Intermediate Level Transuranic Storage Facility (ILTSF). ILTSF is the designated location for all remote-handled waste. In this table, the designation TSA therefore means all of the Transuranic Storage Area except ILTSF. Locations outside of the Transuranic Storage Area, such as Pad A and the Burial Ground Pit Air Support Building, are not considered here.

is stored at one location, the numbers of shipments for the locations add up to the total number, and their volumes and activities add up to the total volume and activity, and the same is true for the breakdown by waste generator.

Table 3 shows a tally of the various containers in the data base. There are diverse potential uses for this information, so the raw counts are simply presented, and not reduced to summaries. 
Table 3. Tally of containers of different types and volumes.

Arranged by Volume, then by Type

\begin{tabular}{|c|c|c|c|c|c|}
\hline Type & Volume $^{b}$ & Number & Iype $^{a}$ & Volume $^{b}$ & Number \\
\hline BLM & $1.0 \mathrm{~F}$ & 1 & 0 & $112.0 \mathrm{~F}$ & 1 \\
\hline$B X C$ & $1.0 \mathrm{~F}$ & 1 & BIN & $115.0 \mathrm{~F}$ & 18 \\
\hline $\begin{array}{l}0 \\
B X C\end{array}$ & $\begin{array}{l}1.0 \mathrm{~F} \\
2.0 \mathrm{~F}\end{array}$ & 29 & $\begin{array}{l}\text { BIN } \\
\text { BXM }\end{array}$ & $\begin{array}{l}120.0 \mathrm{~F} \\
1200 \mathrm{~F}\end{array}$ & 528 \\
\hline BXW & $2.0 \mathrm{~F}$ & 2 & BXW & $120.0 \mathrm{~F}$ & $\begin{array}{r}25 \\
7\end{array}$ \\
\hline BLM & $3.0 \mathrm{~F}$ & $i$ & BXW & $121.0 \mathrm{~F}$ & 137 \\
\hline BXW & $3.0 \mathrm{~F}$ & $i$ & BXW & $128.0 \mathrm{~F}$ & \\
\hline I & $5.0 \mathrm{~F}$ & 30 & BXW & $130.0 \mathrm{~F}$ & 7 \\
\hline BLM & $7.0 \mathrm{~F}$ & 189 & BXW & $144.0 \mathrm{~F}$ & 5 \\
\hline $\begin{array}{l}\text { RD5 } \\
\text { RDL }\end{array}$ & $7.0 \mathrm{~F}$ & 4 & $\begin{array}{l}\text { BXW } \\
\text { PYW }\end{array}$ & $145.0 \mathrm{~F}$ & 2 \\
\hline BLM & $8.0 \mathrm{~F}$ & $\begin{array}{r}160 \\
1750\end{array}$ & $\begin{array}{l}B \times W \\
B X W\end{array}$ & $159.0 \mathrm{~F}$ & $\begin{array}{l}2 \\
6\end{array}$ \\
\hline$B \times W$ & $8.0 \mathrm{~F}$ & 1 & BXW & $176.0 \mathrm{~F}$ & \\
\hline RDL & $8.0 \mathrm{~F}$ & 10 & BXW & $188.0 \mathrm{~F}$ & 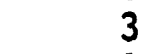 \\
\hline $\begin{array}{l}\text { BLM } \\
\text { BXW }\end{array}$ & $\begin{array}{r}9.0 \mathrm{~F} \\
18.0 \mathrm{~F}\end{array}$ & 3 & $\begin{array}{l}\text { BXW } \\
\text { BXW }\end{array}$ & $212.0 \mathrm{~F}$ & $2^{3}$ \\
\hline 0 & $22.0 \mathrm{~F}$ & 9 & BXW & $272.0 \mathrm{~F}$ & 62 \\
\hline BXW & $25.0 \mathrm{~F}$ & 1 & BXW & $378.0 \mathrm{~F}$ & 4 \\
\hline $\begin{array}{l}\text { BLM } \\
B X W\end{array}$ & $\begin{array}{l}28.0 \mathrm{~F} \\
46.0 \mathrm{~F}\end{array}$ & $\frac{1}{1}$ & $\begin{array}{l}B X W \\
B X W\end{array}$ & $\begin{array}{l}400.0 \mathrm{~F} \\
404.0 \mathrm{~F}\end{array}$ & $\begin{array}{r}11 \\
3\end{array}$ \\
\hline BIN & $48.0 \mathrm{~F}$ & 2 & BXW & $424.0 \mathrm{~F}$ & 2 \\
\hline BLM & $50.0 \mathrm{~F}$ & 1 & BXW & $611.0 \mathrm{~F}$ & 5 \\
\hline $\begin{array}{l}\text { BLM } \\
\text { BXW }\end{array}$ & $\begin{array}{l}55.0 \mathrm{~F} \\
56.0 \mathrm{~F}\end{array}$ & $\begin{array}{r}36 \\
182\end{array}$ & $\begin{array}{l}\text { BXW } \\
\text { BXW }\end{array}$ & $\begin{array}{l}617.0 \mathrm{~F} \\
636.0 \mathrm{~F}\end{array}$ & $\begin{array}{r}20 \\
2\end{array}$ \\
\hline BXW & $61.0 \mathrm{~F}$ & 4 & 0 & $1.0 \mathrm{~K}$ & 3 \\
\hline BIN & $62.0 \mathrm{~F}$ & 1 & BLM & $2.0 \mathrm{~K}$ & 2 \\
\hline $\begin{array}{l}\text { BXW } \\
\text { BYW }\end{array}$ & $69.0 \mathrm{~F}$ & 5 & 0 & $2.0 \mathrm{~K}$ & \\
\hline $\begin{array}{l}\text { BXW } \\
\text { BXW }\end{array}$ & $\begin{array}{l}72.0 \mathrm{~F} \\
84.0 \mathrm{~F}\end{array}$ & $\begin{array}{l}5 \\
9\end{array}$ & $\begin{array}{l}B L M \\
0\end{array}$ & $\begin{array}{l}5.0 \mathrm{~K} \\
5.0 \mathrm{~K}\end{array}$ & 3 \\
\hline$B X$ & $93.0 \mathrm{~F}$ & 13 & BLM & $30.0 \hat{k}$ & $1070^{\circ}$ \\
\hline 0 & $96.0 \mathrm{~F}$ & 25 & BLM & $35.0 \mathrm{~K}$ & \\
\hline & $96.0 \mathrm{~F}$ & 85 & BLM & $55.0 \mathrm{~K}$ & 139259 \\
\hline BX & $106.0 \mathrm{~F}$ & 88 & RD5 & $55.0 \mathrm{~K}$ & \\
\hline BI & $108.0 \mathrm{~F}$ & 1 & BLM & $83.0 \mathrm{~K}$ & 3953 \\
\hline & $108.0 \mathrm{~F}$ & 8 & BLM & $86.0 \mathrm{~K}$ & 55 \\
\hline & $111.0 \mathrm{~F}$ & 24 & BLM & $100.0 \mathrm{~K}$ & 77 \\
\hline & $112.0 \mathrm{~F}$ & 331 & BXM & $355.0 \mathrm{~K}$ & 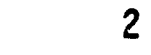 \\
\hline & & & & & \\
\hline
\end{tabular}


Table 3. (Cont.)

Arranged by Type, then by Volume

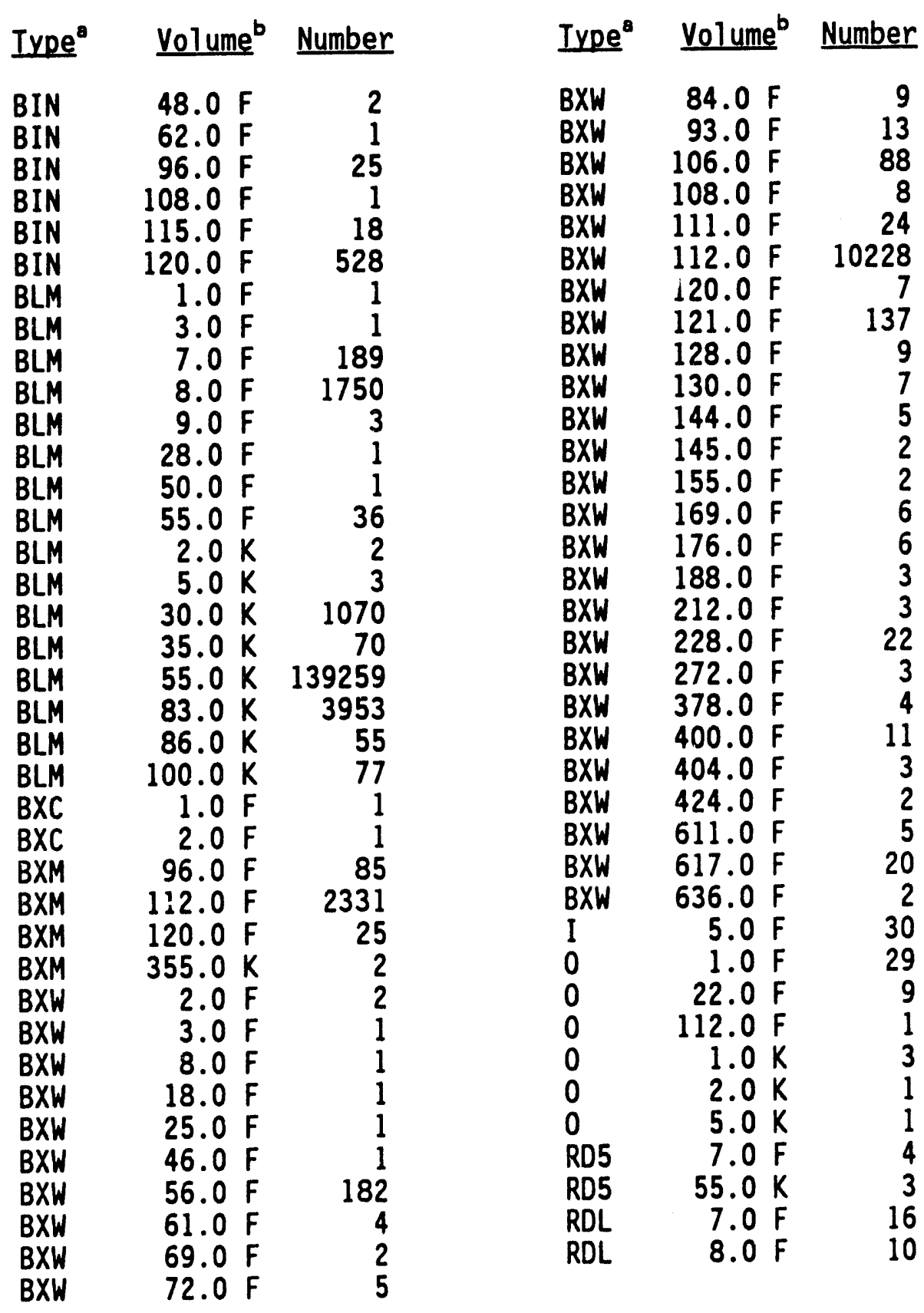

a. The codes for container type are BIN for bin, BLM for metal barre1, BXC for cardboard box, BXM for metal box, BXW for wooden box, I for insert, 0 for other, RDL for 55 gal metal barrel with liner, and RD5 for 55 gal metal barrel without 1 iner.

b. F means cubic foot, $K$ means gallon. 
The above summarizes the data to be analyzed. Conceptually, the analysis method is as follows. Assume that each shipment is homogeneous, and divide the shipment activity by the shipment volume to obtain the "normalized activity" $(\mathrm{mCi} / \mathrm{cu}-\mathrm{ft})$ for each shipment. From this, obtain the empirical distribution of normalized activity (mCi/cu-ft), reflecting the fact that different shipments have different activities and different volumes. One could work with the gallon or the cubic foot as the basic normalizing unit, but we choose the cubic foot. The two are related by $1 \mathrm{gal}=231 / 1728 \mathrm{cu}-\mathrm{ft}=0.1337 \mathrm{cu}-\mathrm{ft}$.

This approach yields the correct overall mean activity per cubic foot. It underestimates the variance somewhat, because it uses the assumption that each shipment is perfectly homogeneous. See Appendix B for some examples of the degree of underestimation of the variance. In those examples the variance is underestimated by $1 / 10$ to $1 / 3$; this corresponds to underestimating the standard deviation by from $5 \%$ to $20 \%$. No way has been found, however, to improve on the method used here, based on the RWMIS data as it is.

To carry out this analysis, software was written in the dBASE language to write, for each shipment, the total container volume and the current (decayed) activity of each nuclide that is present in the shipment. This text file was read by a Fortran program to extract the relevant information for the major nuclides, and for classes of nuclides such as transuranic (TRU) nuclides or $\alpha$-emitters. Finally the statistical package SAS was used to analyze this information and to approximate the empirical distribution.

To simulate a container using this method, draw a random normalized activity (mCi/cu-ft) from the empirical distribution, then multiply that normalized activity by the number of cubic feet in the container. The result is the simulated activity ( $\mathrm{mC} i$ ) for the container. In the same manner, one can simulate the mass (mg) of a fissionable nuclide or class of nuclides, or the committed effective dose equivalent (Mrem) from inhalation. 


\section{RESULTS}

\section{TABULATEd Empirical DISTRIBUTIONS}

The empirical distributions are given for a number of nuclides and classes of nuclides and containers. Several classes of nuclides are mentioned below: transuranic (TRU), non-TRU, $\alpha$-emitters, $\beta / \gamma$-emitters, and fissionable nuclides. Table 1 shows which nuclides fall into which classes. For nearly all classes, the empirical distribution is expressed in terms of $\mathrm{mCi} / \mathrm{cu}-\mathrm{ft}$, and reflects the variation between shipments. For fissionable nuclides, the empirical distribution is expressed in $\mathrm{mg} / \mathrm{cu}-\mathrm{ft}$. Finally, the committed effective dose equivalent from inhalation is considered, and its empirical distribution is expressed in Mrem/cu-ft. The empirical distribution is given for:

- All the nuclides combined.

- The transuranic (TRU) nuclides as a class. The total activity of the TRU nuclides in a shipment is found, the resulting normalized activity in $\mathrm{mCi} / \mathrm{cu}-\mathrm{ft}$ is found for each shipment, and the empirical distribution of this normalized activity is presented. The standard definition of a TRU nuclide is used: atomic number $>92$, and half- 7 ife $>20$ years.

- The non-TRU nuclides as a class.

- The $\alpha$-emitting nuclides as a class.

- The $\beta / \gamma$-emitting nuclides as a class.

- Any nuclide with present total activity greater than $2 \mathrm{Ci}$. The cutoff of $2 \mathrm{Ci}$ was chosen because the generation of daughter products was fairly easy to program in dBASE for inuclides that would result in activity of $2 \mathrm{Ci}$ or more, but would have been cumbersome if certain other nuclides had been included. Because the generation of daughters was not programmed for daughters with less than $2 \mathrm{Ci}$, it is not meaningful to consider the 
empirical distributions of those nuclides.

Each major waste generator as a class. For example, the empirical distribution of normalized activity ( $\mathrm{mCi} / \mathrm{cu}-\mathrm{ft}$ ) from Rocky Flats is given. Seven waste generators are treated in this way. For each such generator, three empirical distributions are given, for the total normalized activity, the TRU normalized activity, and the non-TRU normalized activity.

- Two storage locations, as classes: the Intermediate-Level Transuranic Storage Facility (ILTSF) and everywhere else at the Transuranic Storage Area (TSA). The reason for this dichotomy is that all remote-handled waste is required to be stored at the ILTSF, while the waste everywhere else must be contact-handled waste. For each of these two locations, three empirical distributions are given, for total normalized activity, TRU normaiized activity, and non-TRU normalized activity.

- Fissionable nuclides, as a class. These empirical distributions are expressed as $\mathrm{mg} / \mathrm{cu}-\mathrm{ft}$, because criticality calculations usually use grams rather than curies. The relation between grams and curies is: grams = 2.798E-6 $\times$ (activity in $\mathrm{C} i$ ) $\times$ (half-1ife in years) $\times$ (atomic mass). Empirical distributions are given for: the total fissionable mass, as a class; the mass of the fissionable TRU nuclides, as a class; the mass of the fissionable non-TRU nuclides, as a class; and the mass of individual fissionable nuclides with total decayed activity $>2 \mathrm{Ci}$.

- The total inhalation committed effective dose equivalent, expressed as Mrem/cu-ft. The dose conversion factors, for converting curies to rems, are listed in Appendix $A$.

These empirical distributions are presented in Tables 4 through 11 . The use of these tables is explained in the following sections. 


\section{How to Read the Tables}

The empirical distributions are discrete rather than continuous, because they are based on the normalized activities of a finite number of shipments. If only a few shipments contain the class of nuclides under consideration, the simplest way to present the empirical distribution is to 1 ist the normalized activities and their probabilities. This is done for a few cases in Table 10, and is explained in more detail below. On the other hand, for a class of nuclides that occurs in many (but not al1) shipments the empirical distribution can be approximated by two parts: a point probability at zero, and a continuous distribution covering the range of normalized activities greater than zero. The continuous portion can be summarized by the quantiles: the 10\% point, the $20 \%$ point, etc. Because the distribution is typically skewed, with a few very large values in the right tail, we show more detail in the uppermost quantiles, listing the $98 \%, 99.5 \%$, and $99.9 \%$ points, as well as the absolute maximum.

Nearly all the results in Tables 4 through 11 are presented by giving three numbers, which must be combined:

(1) A, a normalized activity, in units $\mathrm{mCi} / \mathrm{cu}-\mathrm{ft}$ in Tables 4 through 7 , in $\mathrm{mg} / \mathrm{cu}-\mathrm{ft}$ in Tables 8 and 9 , and in Mrem/cu-ft in Table 11

(2) $P(A)$, the cumulative probability that the random normalized activity is less than $A$, conditional on the activity being greater than zero

(3) $p_{\text {pos }}$, the probability that a random cubic foot of waste will contain non-zero activity of the type of interest.

The probability that a random cubic foot of waste will have activity less than or equal to $A$ is given by

$\left(1-p_{\text {pos }}\right)+p_{\text {pos }} \times P(A)$

The first of the two added terms is the probability that the random cubic foot has no activity from the class of interest, and the second term is the probability that the activity is positive but at most $A$. 
Table 4. Distributions of major $\mathrm{classes}$ of nuclides, $\mathrm{mCi} / \mathrm{cu}-\mathrm{ft}$.

\begin{tabular}{|c|c|c|c|c|c|}
\hline A11 & TRU & Non-TRU & $\alpha$-Emitters & $\beta / \gamma$-Emitters & \multirow[b]{2}{*}{$\begin{array}{l}\text { Conditional } \\
\text { Cumulative } \\
\text { Probability }\end{array}$} \\
\hline \multicolumn{5}{|c|}{ Normalized Activity (mCi/cu-ft) } & \\
\hline $\begin{array}{c}0 . \\
0.449 \\
2.25 \\
11.8 \\
24.7 \\
34.6 \\
61.2 \\
91.0 \\
188 . \\
430 . \\
1020 . \\
2920 . \\
7510 . \\
390000 .\end{array}$ & $\begin{array}{c}0 . \\
0.135 \\
0.556 \\
3.68 \\
7.71 \\
11.5 \\
19.7 \\
41.3 \\
74.9 \\
223 . \\
691 . \\
1830 . \\
4370 . \\
30300 .\end{array}$ & $\begin{array}{c}0 . \\
0.278 \\
0.727 \\
5.32 \\
13.2 \\
20.0 \\
30.1 \\
44.8 \\
81.3 \\
178 . \\
456 . \\
906 . \\
3680 . \\
389000 .\end{array}$ & $\begin{array}{c}0 . \\
0.136 \\
0.567 \\
3.72 \\
7.78 \\
11.5 \\
20.0 \\
41.7 \\
74.8 \\
223 . \\
689 . \\
1810 . \\
4370 . \\
71400 .\end{array}$ & $\begin{array}{c}0 . \\
0.291 \\
0.872 \\
5.47 \\
13.6 \\
20.4 \\
30.7 \\
45.6 \\
83.0 \\
179 . \\
459 . \\
914 . \\
3720 . \\
389000 .\end{array}$ & $\begin{array}{l}0.0 \\
0.10 \\
0.20 \\
0.30 \\
0.40 \\
0.50 \\
0.60 \\
0.70 \\
0.80 \\
0.90 \\
0.98 \\
0.995 \\
0.999 \\
1.000\end{array}$ \\
\hline \multicolumn{5}{|c|}{$P($ Activity $>0)$} & \\
\hline 1.00000 & 0.99198 & 0.99771 & 0.99863 & 0.98140 & \\
\hline
\end{tabular}


Table 5. Distributions of individual nuclides, $\mathrm{mCi} / \mathrm{cu}-\mathrm{ft}$.

\begin{tabular}{|c|c|c|c|c|c|}
\hline Am-241 & $\mathrm{Ba}-137 \mathrm{~m}$ & $\mathrm{Bi}-212$ & $\mathrm{Ce}-144$ & $\mathrm{Cm}-244^{\circ}$ & \multirow{2}{*}{$\begin{array}{l}\text { Cond. Cum. } \\
\text { Probability }\end{array}$} \\
\hline \multicolumn{5}{|c|}{ Normalized Activity (mCi/cu-ft) } & \\
\hline $\begin{array}{c}0 . \\
0.0143 \\
0.0363 \\
0.216 \\
0.500 \\
0.847 \\
1.16 \\
2.97 \\
27.1 \\
120 . \\
465 . \\
814 . \\
1300 . \\
3370 .\end{array}$ & $\begin{array}{c}0 . \\
0.255 \\
1.23 \\
1.25 \\
1.28 \\
1.31 \\
1.37 \\
1.38 \\
1.40 \\
1.47 \\
109 . \\
760 . \\
6460 . \\
93100 .\end{array}$ & $\begin{array}{l}0 . \\
0.269 \\
0.317 \\
0.443 \\
0.485 \\
0.541 \\
0.738 \\
0.789 \\
0.941 \\
9.22 \\
16.1 \\
23.9 \\
23.9 \\
23.9\end{array}$ & $\begin{array}{l}0 . \\
0.00140 \\
0.00176 \\
0.00210 \\
0.00242 \\
0.00647 \\
0.0374 \\
0.422 \\
0.925 \\
35.5 \\
790 . \\
3560 . \\
3560 . \\
3560 .\end{array}$ & $\begin{array}{l}0 . \\
0.000000 \\
0.000001 \\
0.000151 \\
0.000151 \\
0.000944 \\
0.0378 \\
2.35 \\
9.47 \\
12.2 \\
12.2 \\
27.4 \\
71400 . \\
71400 .\end{array}$ & $\begin{array}{l}0.0 \\
0.10 \\
0.20 \\
0.30 \\
0.40 \\
0.50 \\
0.60 \\
0.70 \\
0.80 \\
0.90 \\
0.98 \\
0.995 \\
0.999 \\
1.000\end{array}$ \\
\hline \multicolumn{5}{|c|}{$P($ Activity $>0)$} & \multirow{4}{*}{$\begin{array}{l}\text { Cond. Cum. } \\
\text { Probability }\end{array}$} \\
\hline 0.9592 & 0.0224 & 0.00609 & 0.000175 & 0.00213 & \\
\hline $\mathrm{Co}-60$ & Cs -134 & Cs -137 & $\mathrm{Kr}-85$ & MFP & \\
\hline \multicolumn{5}{|c|}{ Normalized Activity $(\mathrm{mCi} / \mathrm{cu}-\mathrm{ft})$} & \\
\hline $\begin{array}{l}0 . \\
0.000176 \\
0.000176 \\
0.000176 \\
0.000176 \\
0.000176 \\
0.000176 \\
0.000176 \\
0.000176 \\
17.7 \\
2680 . \\
2940 . \\
2940 . \\
2940 .\end{array}$ & $\begin{array}{c}0 . \\
1.30 \\
1.73 \\
2.57 \\
2.59 \\
3.01 \\
4.15 \\
19.2 \\
103 . \\
650 . \\
3050 . \\
10300 . \\
10300 . \\
10300 .\end{array}$ & $\begin{array}{c}0 . \\
0.000020 \\
0.000233 \\
0.00109 \\
0.00181 \\
0.00181 \\
0.00181 \\
0.244 \\
1.49 \\
98.3 \\
1630 . \\
13000 . \\
74000 . \\
93100 .\end{array}$ & $\begin{array}{l}0 . \\
522 . \\
522 . \\
522 . \\
522 . \\
850 . \\
850 . \\
850 . \\
850 . \\
850 . \\
850 . \\
850 . \\
850 . \\
850 .\end{array}$ & $\begin{array}{c}0 . \\
2.43 \\
2.44 \\
2.53 \\
2.61 \\
2.67 \\
2.73 \\
2.75 \\
2.78 \\
2.82 \\
113 . \\
329 . \\
934 . \\
2700 .\end{array}$ & $\begin{array}{l}0.0 \\
0.10 \\
0.20 \\
0.30 \\
0.40 \\
0.50 \\
0.60 \\
0.70 \\
0.80 \\
0.90 \\
0.98 \\
0.995 \\
0.999 \\
1.000\end{array}$ \\
\hline \multicolumn{5}{|c|}{$P($ Activity $>0)$} & \\
\hline 0.000371 & 0.000143 & 0.00268 & .00000437 & 0.0204 & \\
\hline
\end{tabular}


Table 5. (Cont.)

\begin{tabular}{|c|c|c|c|c|c|}
\hline $\mathrm{Ni}-63^{\circ}$ & $\mathrm{Pb}-212$ & $\mathrm{Pm}-147^{a}$ & $P_{0}-212$ & $P_{0}-216$ & \multirow{2}{*}{$\begin{array}{l}\text { Cond. Cum. } \\
\text { Probability }\end{array}$} \\
\hline \multicolumn{5}{|c|}{ Normalized Activity (mCi/cu-ft) } & \\
\hline $\begin{array}{l}0 . \\
0.000129 \\
0.000129 \\
0.000129 \\
0.000129 \\
0.000129 \\
0.000129 \\
0.000129 \\
0.000129 \\
0.000129 \\
0.000129 \\
589 . \\
589 . \\
589 .\end{array}$ & $\begin{array}{l}0 . \\
0.269 \\
0.317 \\
0.443 \\
0.485 \\
0.541 \\
0.738 \\
0.789 \\
0.941 \\
9.22 \\
16.1 \\
23.9 \\
23.9 \\
23.9\end{array}$ & $\begin{array}{l}0 . \\
507 . \\
507 . \\
507 . \\
507 . \\
1530 . \\
1530 . \\
1530 . \\
3180 . \\
3180 . \\
3180 . \\
3180 . \\
3180 . \\
3180 .\end{array}$ & $\begin{array}{l}0 . \\
0.172 \\
0.203 \\
0.284 \\
0.311 \\
0.347 \\
0.473 \\
0.506 \\
0.603 \\
5.91 \\
10.3 \\
15.3 \\
15.3 \\
15.3\end{array}$ & $\begin{array}{l}0 . \\
0.269 \\
0.317 \\
0.443 \\
0.485 \\
0.541 \\
0.738 \\
0.789 \\
0.941 \\
9.22 \\
16.1 \\
23.9 \\
23.9 \\
23.9\end{array}$ & $\begin{array}{l}0.0 \\
0.10 \\
0.20 \\
0.30 \\
0.40 \\
0.50 \\
0.60 \\
0.70 \\
0.80 \\
0.90 \\
0.98 \\
0.995 \\
0.999 \\
1.000\end{array}$ \\
\hline \multicolumn{5}{|c|}{$P($ Activity $>0)$} & \multirow{4}{*}{$\begin{array}{l}\text { Cond. Cum. } \\
\text { Probability }\end{array}$} \\
\hline 0.000319 & 0.00610 & 0.00000759 & 0.00610 & 0.00610 & \\
\hline $\operatorname{Pr}-144$ & $P u-238$ & $P u=239$ & $P u-240$ & $\mathrm{Pu}-241$ & \\
\hline \multicolumn{5}{|c|}{ Normalized Activity $(\mathrm{mCi} / \mathrm{cu}-\mathrm{ft})$} & \\
\hline $\begin{array}{l}0 . \\
0.00141 \\
0.00177 \\
0.00211 \\
0.00243 \\
0.00650 \\
0.0376 \\
0.424 \\
0.929 \\
35.7 \\
793 . \\
3570 . \\
3570 . \\
3570 .\end{array}$ & $\begin{array}{c}0 . \\
0.00242 \\
0.0106 \\
0.0862 \\
0.158 \\
0.211 \\
0.323 \\
0.559 \\
1.01 \\
3.22 \\
132 . \\
1670 . \\
4360 . \\
30300 .\end{array}$ & $\begin{array}{c}0 . \\
0.0684 \\
0.186 \\
1.03 \\
3.66 \\
6.04 \\
7.82 \\
12.3 \\
20.9 \\
43.9 \\
121 . \\
195 . \\
729 . \\
1600 .\end{array}$ & $\begin{array}{c}0 . \\
0.0210 \\
0.0521 \\
0.366 \\
0.981 \\
1.50 \\
1.92 \\
3.04 \\
5.23 \\
10.9 \\
29.1 \\
47.1 \\
195 . \\
1780 .\end{array}$ & $\begin{array}{c}0 . \\
0.290 \\
0.841 \\
6.70 \\
15.2 \\
21.3 \\
31.2 \\
47.3 \\
85.6 \\
181 . \\
460 . \\
905 . \\
3580 . \\
25100 .\end{array}$ & $\begin{array}{l}0.0 \\
0.10 \\
0.20 \\
0.30 \\
0.40 \\
0.50 \\
0.60 \\
0.70 \\
0.80 \\
0.90 \\
0.98 \\
0.995 \\
0.999 \\
1.000\end{array}$ \\
\hline \multicolumn{5}{|c|}{$P($ Activity $>0)$} & \\
\hline 0.000175 & 0.9694 & 0.99187 & 0.9679 & 0.9535 & \\
\hline
\end{tabular}


Tabie 5. (Cont.)

\begin{tabular}{|c|c|c|c|c|c|}
\hline $\mathrm{Ra}-224$ & $\mathrm{Rn}-220$ & Sr-90 & Th-228 & T1-208 & \multirow{2}{*}{$\begin{array}{l}\text { Cond. Cum. } \\
\text { Probability }\end{array}$} \\
\hline \multicolumn{5}{|c|}{ Normalized Activity (mCi/cu-ft) } & \\
\hline $\begin{array}{l}0 . \\
0.269 \\
0.317 \\
0.443 \\
0.485 \\
0.541 \\
0.738 \\
0.789 \\
0.941 \\
9.22 \\
16.1 \\
23.9 \\
23.9 \\
23.9\end{array}$ & $\begin{array}{l}0 . \\
0.269 \\
0.317 \\
0.443 \\
0.485 \\
0.541 \\
0.738 \\
0.789 \\
0.941 \\
9.22 \\
16.1 \\
23.9 \\
23.9 \\
23.9\end{array}$ & $\begin{array}{r}0 . \\
278 . \\
515 . \\
644 . \\
773 . \\
773 . \\
901 . \\
1620 . \\
5730 . \\
11800 . \\
73600 . \\
92800 . \\
92800 . \\
92800 .\end{array}$ & $\begin{array}{l}0 . \\
0.269 \\
0.317 \\
0.443 \\
0.485 \\
0.541 \\
0.738 \\
0.789 \\
0.941 \\
9.22 \\
16.1 \\
23.9 \\
23.9 \\
23.9\end{array}$ & $\begin{array}{l}0 . \\
0.0966 \\
0.114 \\
0.159 \\
0.174 \\
0.195 \\
0.265 \\
0.284 \\
0.338 \\
3.31 \\
5.80 \\
8.59 \\
8.59 \\
8.59\end{array}$ & $\begin{array}{l}0.0 \\
0.10 \\
0.20 \\
0.30 \\
0.40 \\
0.50 \\
0.60 \\
0.70 \\
0.80 \\
0.90 \\
0.98 \\
0.995 \\
0.999 \\
1.000\end{array}$ \\
\hline \multicolumn{5}{|c|}{$P($ Activity $>0)$} & \multirow{4}{*}{$\begin{array}{l}\text { Cond. Cum. } \\
\text { Probabilit }\end{array}$} \\
\hline 0.00610 & 0.00610 & 0.000138 & 0.00610 & 0.00610 & \\
\hline$U-232$ & $U-233$ & $U-234$ & Unid. $\beta / \gamma^{a}$ & $Y-90$ & \\
\hline \multicolumn{5}{|c|}{ Normalized Activity (mCi/cu-ft) } & \\
\hline $\begin{array}{l}0 . \\
0.262 \\
0.309 \\
0.432 \\
0.473 \\
0.527 \\
0.720 \\
0.769 \\
0.917 \\
9.09 \\
15.8 \\
23.5 \\
23.5 \\
23.5\end{array}$ & $\begin{array}{l}0 . \\
0.000162 \\
0.000485 \\
0.000612 \\
0.000705 \\
0.00140 \\
0.0368 \\
0.846 \\
20.7 \\
47.8 \\
198 . \\
215 . \\
319 . \\
330 .\end{array}$ & $\begin{array}{l}0 . \\
0.000000 \\
0.000000 \\
0.000004 \\
0.000007 \\
0.000010 \\
0.000013 \\
0.000019 \\
0.000034 \\
0.000125 \\
0.00727 \\
0.145 \\
0.440 \\
1.81\end{array}$ & $\begin{array}{l}0 . \\
47.6 \\
63.4 \\
63.4 \\
209 . \\
209 . \\
313 . \\
319 . \\
319 . \\
731 . \\
731 . \\
731 . \\
731 . \\
731 .\end{array}$ & $\begin{array}{c}0 . \\
1.21 \\
1.21 \\
1.26 \\
1.29 \\
1.33 \\
1.36 \\
1.37 \\
1.39 \\
1.46 \\
71.6 \\
644 . \\
7510 . \\
92800 .\end{array}$ & $\begin{array}{l}0.0 \\
0.10 \\
0.20 \\
0.30 \\
0.40 \\
0.50 \\
0.60 \\
0.70 \\
0.80 \\
0.90 \\
0.98 \\
0.995 \\
0.999 \\
1.000\end{array}$ \\
\hline \multicolumn{5}{|c|}{$P($ Activity $>0)$} & \\
\hline 0.00610 & 0.0245 & 0.9731 & 0.0000199 & 0.0206 & \\
\hline
\end{tabular}


Table 6. Distributions of activity from major generators, mCi/cu-ft.

\begin{tabular}{|c|c|c|c|c|c|c|}
\hline \multicolumn{3}{|c|}{ ALE (Argonne Lab East) } & \multicolumn{3}{|c|}{ ANL (Argonne Lab West) } & \multirow[b]{2}{*}{$\begin{array}{l}\text { Cond. Cum. } \\
\text { Probability }\end{array}$} \\
\hline \multicolumn{6}{|c|}{ Normalized Activity $(\mathrm{mCi} / \mathrm{cu}-\mathrm{ft})$} & \\
\hline $\begin{array}{c}0 . \\
5.45 \\
5.55 \\
5.74 \\
5.89 \\
6.20 \\
6.29 \\
7.26 \\
12.5 \\
35.6 \\
712 . \\
1910 . \\
22000 . \\
26300 .\end{array}$ & $\begin{array}{c}0 . \\
0.482 \\
0.528 \\
0.567 \\
0.651 \\
0.740 \\
0.854 \\
2.29 \\
8.55 \\
18.4 \\
183 . \\
285 . \\
1010 . \\
1921 .\end{array}$ & $\begin{array}{c}0 . \\
0.999 \\
4.87 \\
4.94 \\
5.07 \\
5.22 \\
5.46 \\
5.50 \\
5.57 \\
5.87 \\
672 . \\
1960 . \\
21100 . \\
25300 .\end{array}$ & $\begin{array}{c}0 . \\
9.42 \\
33.2 \\
90.8 \\
191 . \\
491 . \\
647 . \\
808 . \\
1070 . \\
1630 . \\
45800 . \\
299000 . \\
390000 . \\
390000 .\end{array}$ & $\begin{array}{c}0 . \\
2.74 \\
14.7 \\
40.0 \\
99.3 \\
195 . \\
496 . \\
646 . \\
819 . \\
1130 . \\
1770 . \\
2320 . \\
2540 . \\
2540 .\end{array}$ & $\begin{array}{c}0 . \\
0.00231 \\
0.0166 \\
0.0398 \\
0.0476 \\
0.0505 \\
0.0659 \\
0.993 \\
23.9 \\
762 . \\
45800 . \\
299000 . \\
389000 . \\
389000 .\end{array}$ & $\begin{array}{l}0.0 \\
0.10 \\
0.20 \\
0.30 \\
0.40 \\
0.50 \\
0.60 \\
0.70 \\
0.80 \\
0.90 \\
0.98 \\
0.995 \\
0.999 \\
1.000\end{array}$ \\
\hline \multicolumn{6}{|c|}{$P($ Activity $>0)$} & \multirow{5}{*}{$\begin{array}{l}\frac{\text { non-TRU }}{\text { Cond. Cum. }} \\
\text { Probability }\end{array}$} \\
\hline 0.0236 & 0.0236 & 0.0230 & 0.000670 & 0.000670 & 0.000659 & \\
\hline$\frac{\mathrm{BET}(\mathrm{Be})}{\mathrm{A} 11}$ & $\frac{\text { s Atomic }}{\text { TRU }}$ & Ower Lab) & $\frac{\text { CPP (Chen }}{\text { non-TRU }}$ & $\frac{\text { Processir }}{A 11}$ & $\frac{\text { ng } P(a n t)}{T R U}$ & \\
\hline \multicolumn{6}{|c|}{ Normalized Activity $(\mathrm{mC} i / \mathrm{cu}-\mathrm{ft})$} & \\
\hline $\begin{array}{c}0 . \\
14.5 \\
20.4 \\
27.8 \\
33.3 \\
38.0 \\
49.7 \\
56.8 \\
69.2 \\
249 . \\
344 . \\
510 . \\
2630 . \\
55900 .\end{array}$ & $\begin{array}{c}0 . \\
1.26 \\
1.26 \\
1.26 \\
1.26 \\
1.26 \\
1.26 \\
1.26 \\
60.6 \\
2570 . \\
2570 . \\
2570 . \\
2570 . \\
2570 .\end{array}$ & $\begin{array}{c}0 . \\
14.5 \\
20.4 \\
27.8 \\
33.3 \\
38.0 \\
49.7 \\
56.8 \\
69.2 \\
249 . \\
344 . \\
510 . \\
510 . \\
54800 .\end{array}$ & $\begin{array}{c}0 . \\
0.428 \\
0.581 \\
0.581 \\
0.872 \\
0.872 \\
0.872 \\
1.23 \\
100 . \\
2920 . \\
5310 . \\
22800 . \\
22800 . \\
22800 .\end{array}$ & $\begin{array}{l}0 . \\
0.195 \\
0.260 \\
0.260 \\
0.391 \\
0.391 \\
0.391 \\
0.997 \\
1.95 \\
14.8 \\
2920 . \\
2920 . \\
2920 . \\
2920 .\end{array}$ & $\begin{array}{c}0 . \\
0.233 \\
0.321 \\
0.321 \\
0.321 \\
0.481 \\
0.481 \\
0.481 \\
0.483 \\
3070 . \\
5120 . \\
22800 . \\
22800 . \\
22800 .\end{array}$ & \\
\hline & & & - & & & \\
\hline & .0000815 & .00002 & 0.0 & 52 & 0.0 & \\
\hline
\end{tabular}


Table 6. (Cont.)

\begin{tabular}{|c|c|c|c|c|c|c|}
\hline \multicolumn{3}{|c|}{ MRC (Mound Laboratory) } & \multicolumn{3}{|c|}{ RFO (Rocky Flats) } & \multirow{3}{*}{$\begin{array}{l}\text { Cond. Cum. } \\
\text { Probability }\end{array}$} \\
\hline A11 & TRU & non-TRU & Al1 & TRU & non-TRU & \\
\hline \multicolumn{6}{|c|}{ Normalized Activity $(\mathrm{mC} / \mathrm{cu}-\mathrm{ft})$} & \\
\hline $\begin{array}{c}0 . \\
5.76 \\
13.5 \\
26.5 \\
38.2 \\
59.0 \\
82.9 \\
143 . \\
301 . \\
1090 . \\
4130 . \\
7820 . \\
8650 . \\
31500 .\end{array}$ & $\begin{array}{c}0 . \\
j .76 \\
13.1 \\
22.9 \\
36.3 \\
58.6 \\
79.7 \\
138 . \\
288 . \\
1050 . \\
3970 . \\
7560 . \\
8360 . \\
30300 .\end{array}$ & $\begin{array}{l}0 . \\
0.000960 \\
0.00569 \\
0.220 \\
0.828 \\
1.47 \\
2.70 \\
5.42 \\
12.5 \\
34.6 \\
153 . \\
274 . \\
290 . \\
1170 .\end{array}$ & $\begin{array}{c}0 . \\
0.416 \\
0.948 \\
10.2 \\
21.4 \\
30.3 \\
52.3 \\
104 . \\
206 . \\
446 . \\
961 . \\
1610 . \\
4700 . \\
10100 .\end{array}$ & $\begin{array}{c}0 . \\
0.129 \\
0.308 \\
2.82 \\
6.34 \\
9.19 \\
15.8 \\
27.9 \\
74.8 \\
228 . \\
602 . \\
1010 . \\
1490 . \\
4260 .\end{array}$ & $\begin{array}{c}0 . \\
0.282 \\
0.657 \\
6.62 \\
14.4 \\
20.6 \\
33.9 \\
53.6 \\
97.0 \\
197 . \\
480 . \\
943 . \\
3590 . \\
7700 .\end{array}$ & $\begin{array}{l}0.0 \\
0.10 \\
0.20 \\
0.30 \\
0.40 \\
0.50 \\
0.60 \\
0.70 \\
0.80 \\
0.90 \\
0.98 \\
0.995 \\
0.999 \\
1.000\end{array}$ \\
\hline \multicolumn{6}{|c|}{$P($ Activity $>0)$} & \\
\hline 0.0602 & 0.0602 & 0.0602 & 0.837 & 0.835 & 0.835 & \\
\hline \multicolumn{7}{|c|}{ WMC (Waste Management Complex) } \\
\hline All & TRU & non-TRU & & & & \\
\hline \multicolumn{3}{|c|}{ Normalized Activity (mCi/cu-ft) } & \multicolumn{2}{|c|}{$\begin{array}{l}\text { Cond. Cum. } \\
\text { Probability }\end{array}$} & & \\
\hline $\begin{array}{l}0 . \\
52.9 \\
83.3 \\
83.3 \\
83.7 \\
84.0 \\
85.0 \\
85.5 \\
85.8 \\
108 . \\
244 . \\
288 . \\
447 . \\
447 .\end{array}$ & $\begin{array}{c}0 . \\
17.6 \\
47.8 \\
54.0 \\
54.0 \\
54.0 \\
54.0 \\
54.1 \\
54.1 \\
62.2 \\
109 . \\
148 . \\
161 . \\
161 .\end{array}$ & $\begin{array}{c}0 . \\
19.7 \\
29.3 \\
29.6 \\
29.9 \\
30.8 \\
31.2 \\
31.5 \\
32.1 \\
45.5 \\
148 . \\
179 . \\
300 . \\
300 .\end{array}$ & $\begin{array}{l}0.0 \\
0.10 \\
0.20 \\
0.30 \\
0.40 \\
0.50 \\
0.60 \\
0.70 \\
0.80 \\
0.90 \\
0.98 \\
0.995 \\
0.999 \\
1.000\end{array}$ & & & \\
\hline \multicolumn{3}{|c|}{$P($ Activity $>0)$} & & & & \\
\hline
\end{tabular}

a. This class occurred in fewer than 20 shipments. The exact distribution is given in Table 10. 


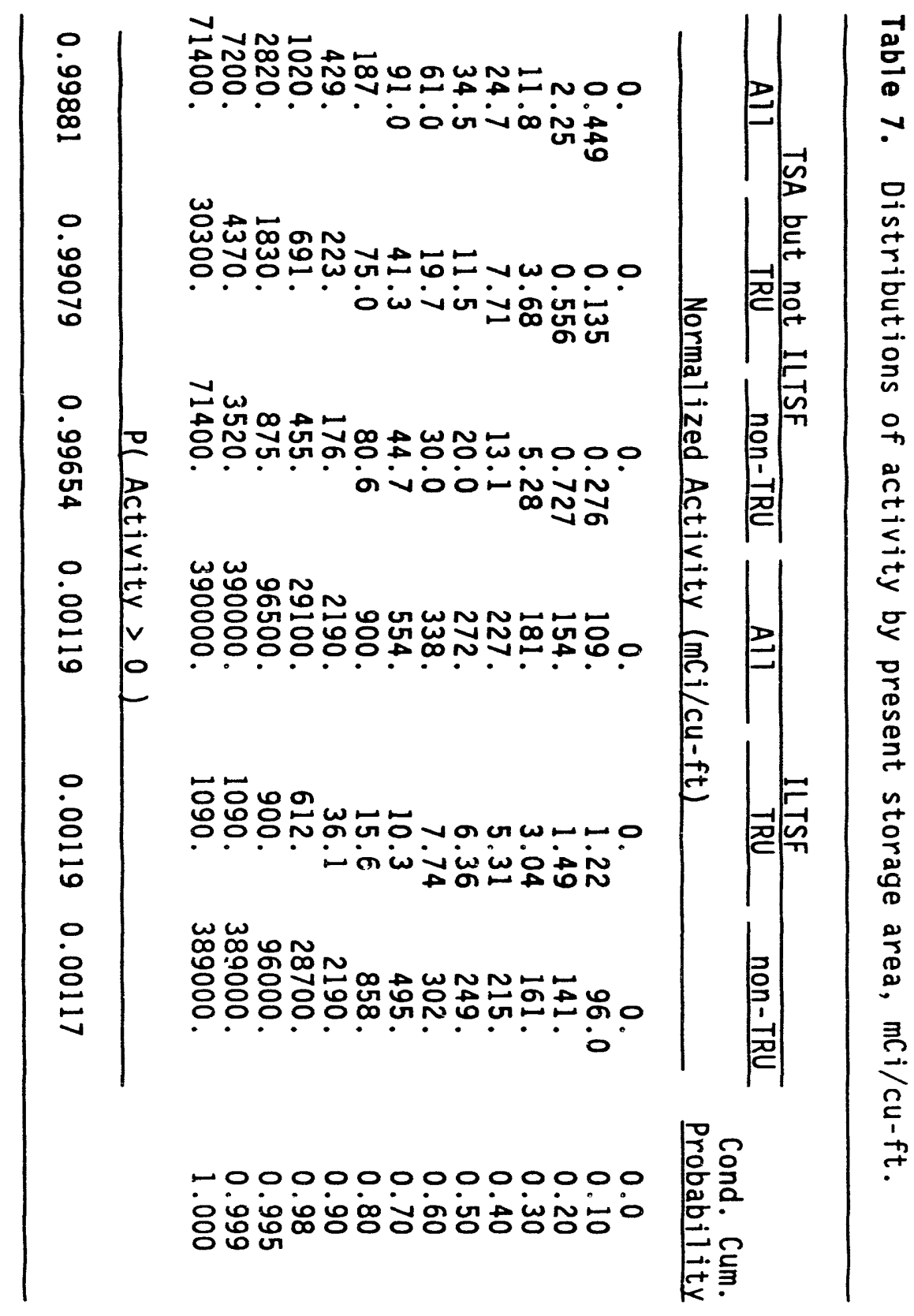


Table 8. Distributions of fissionable nuclides by $\mathrm{class}, \mathrm{mg} / \mathrm{cu}-\mathrm{ft}$.

\begin{tabular}{|c|c|c|c|}
\hline Al1 & TRU & non-TRU & \multirow[b]{2}{*}{$\begin{array}{l}\text { Cond. Cum. } \\
\text { Probability }\end{array}$} \\
\hline \multicolumn{3}{|c|}{ Normalized Activity $(\mathrm{mg} / \mathrm{cu}-\mathrm{ft})$} & \\
\hline $\begin{array}{c}0 . \\
1.50 \\
3.81 \\
22.6 \\
65.9 \\
104 . \\
41 . \\
224 . \\
378 . \\
832 . \\
2200 . \\
5370 . \\
20700 . \\
39800 .\end{array}$ & $\begin{array}{l}0 . \\
0.00479 \\
0.0170 \\
0.109 \\
0.201 \\
0.309 \\
0.509 \\
1.69 \\
12.9 \\
40.4 \\
151 . \\
263 . \\
442 . \\
1770 .\end{array}$ & $\begin{array}{c}0 . \\
0.00286 \\
0.00926 \\
0.0750 \\
0.171 \\
0.284 \\
0.410 \\
0.847 \\
2.34 \\
9.67 \\
88.6 \\
3030 . \\
19700 . \\
38900 .\end{array}$ & $\begin{array}{l}0.0 \\
0.10 \\
0.20 \\
0.30 \\
0.40 \\
0.50 \\
0.60 \\
0.70 \\
0.80 \\
0.90 \\
0.98 \\
0.995 \\
0.999 \\
1.000\end{array}$ \\
\hline \multicolumn{3}{|c|}{$P($ Activity $>0)$} & \\
\hline 0.99863 & 0.99198 & 0.96800 & \\
\hline
\end{tabular}


Table 9. Distributions of individual fissionable nuclides, $\mathrm{mg} / \mathrm{cu}-\mathrm{ft}$.

\begin{tabular}{|c|c|c|c|c|c|}
\hline Am-241 & $\mathrm{Cm}-244{ }^{\circ}$ & $P u-238$ & $P u-239$ & Pu-241 & \multirow{2}{*}{$\begin{array}{l}\text { Cond. Cum. } \\
\text { Probability }\end{array}$} \\
\hline \multicolumn{5}{|c|}{ Normalized Activity $(\mathrm{mg} / \mathrm{cu}-\mathrm{ft})$} & \\
\hline $\begin{array}{l}0 . \\
0.00417 \\
0.0106 \\
0.0631 \\
0.146 \\
0.247 \\
0.339 \\
0.867 \\
7.91 \\
35.1 \\
136 . \\
237 . \\
379 . \\
984 .\end{array}$ & $\begin{array}{l}0 . \\
0.0000000 \\
0.0000000 \\
0.0000019 \\
0.0000019 \\
0.0000117 \\
0.000467 \\
0.0290 \\
0.117 \\
0.151 \\
0.151 \\
0.338 \\
881 . \\
881 .\end{array}$ & $\begin{array}{c}0 . \\
0.000 \\
0.000 \\
0.005 \\
0.005 \\
0.012 \\
0.018 \\
0.032 \\
0.059 \\
0.188 \\
7.69 \\
97.3 \\
254 . \\
1770 .\end{array}$ & $\begin{array}{c}0 . \\
1.10 \\
2.99 \\
16.6 \\
58.9 \\
97.2 \\
126 . \\
198 . \\
336 . \\
708 . \\
1940 . \\
3140 . \\
11700 . \\
25800 .\end{array}$ & $\begin{array}{l}0 . \\
0.00281 \\
0.00816 \\
0.0650 \\
0.147 \\
0.207 \\
0.303 \\
0.459 \\
0.830 \\
1.75 \\
4.46 \\
8.78 \\
34.7 \\
243 .\end{array}$ & $\begin{array}{l}0.0 \\
0.10 \\
0.20 \\
0.30 \\
0.40 \\
0.50 \\
0.60 \\
0.70 \\
0.80 \\
0.90 \\
0.98 \\
0.995 \\
0.999 \\
1.000\end{array}$ \\
\hline & $P(A$ & ctivity & & & \\
\hline 0.9592 & 0.00213 & 0.9694 & 0.99187 & 0.9535 & \\
\hline$U-233$ & & & & & \\
\hline Normalized & tivity $(\mathrm{mg} / \mathrm{cu}$ & $f(t)$ & $\begin{array}{l}\text { Cum. } \\
\text { ability }\end{array}$ & & \\
\hline $\begin{array}{c}0 . \\
0.0169 \\
0.0505 \\
0.0638 \\
0.0734 \\
0.146 \\
3.84 \\
88.1 \\
2160 . \\
4980 . \\
20700 . \\
22400 . \\
33300 . \\
34400 .\end{array}$ & & & $\begin{array}{l}.0 \\
.10 \\
.20 \\
.30 \\
.40 \\
.50 \\
.60 \\
.70 \\
.80 \\
.90 \\
.98 \\
.995 \\
.999 \\
.000\end{array}$ & & \\
\hline
\end{tabular}

0.0245

$P($ Activity $>0)$

a. This nuclide occurred in fewer than 20 shipments. The exact distribution is given in Table 10. 
Table 10. Exact distributions for classes occurring in few shipments.

\begin{tabular}{|c|c|c|c|c|}
\hline \multicolumn{3}{|c|}{$\mathrm{Cm}-244$} & \multicolumn{2}{|c|}{$\mathrm{C}_{0}-60$} \\
\hline $\begin{array}{l}\text { Norm.Act. } \\
(\mathrm{mC} i / \mathrm{cu}-\mathrm{ft})\end{array}$ & $\begin{array}{l}\text { Norm. Act } \\
(\mathrm{mg} / \mathrm{cu}-\mathrm{ft})\end{array}$ & $\begin{array}{l}\text { Cond. Pt. } \\
\text { Probability }\end{array}$ & $\begin{array}{l}\text { Norm.Act. } \\
(\mathrm{mCi} / \mathrm{cu}-\mathrm{ft})\end{array}$ & $\begin{array}{l}\text { Cond. Pt. } \\
\text { Probability }\end{array}$ \\
\hline $\begin{array}{c}0.0000001 \\
0.0000009 \\
0.000151 \\
0.000944 \\
0.00314 \\
0.00873 \\
0.0378 \\
2.35 \\
5.47 \\
9.47 \\
12.2 \\
27.4 \\
36.8 \\
77.3 \\
71400 .\end{array}$ & $\begin{array}{l}0.0000000 \\
0.0000000 \\
0.0000019 \\
0.0000117 \\
0.0000387 \\
0.000108 \\
0.000467 \\
0.0290 \\
0.0676 \\
0.117 \\
0.151 \\
0.338 \\
0.454 \\
0.954 \\
881 .\end{array}$ & $\begin{array}{l}0.14778 \\
0.14778 \\
0.14778 \\
0.14778 \\
0.00003 \\
0.00014 \\
0.10563 \\
0.04926 \\
0.00151 \\
0.14778 \\
0.09852 \\
0.00151 \\
0.00151 \\
0.00151 \\
0.00151\end{array}$ & $\begin{array}{c}0.000176 \\
1.29 \\
6.99 \\
14.0 \\
17.7 \\
19.7 \\
23.1 \\
32.1 \\
43.1 \\
49.6 \\
104 . \\
279 . \\
491 . \\
771 . \\
1500 . \\
2010 . \\
2680 . \\
2880 . \\
2940 .\end{array}$ & $\begin{array}{l}0.84865 \\
0.00589 \\
0.03300 \\
0.00943 \\
0.00943 \\
0.00589 \\
0.00589 \\
0.00589 \\
0.00589 \\
0.00589 \\
0.00589 \\
0.00589 \\
0.00589 \\
0.00589 \\
0.00589 \\
0.00867 \\
0.00867 \\
0.00867 \\
0.00867\end{array}$ \\
\hline \multicolumn{5}{|c|}{$P($ Activity $>0)$} \\
\hline
\end{tabular}


Table 10. (Cont.)

\begin{tabular}{|c|c|c|c|c|c|}
\hline \multicolumn{2}{|c|}{$\mathrm{Kr}-85$} & \multicolumn{2}{|c|}{$\mathrm{Ni}-63$} & \multicolumn{2}{|c|}{$\mathrm{Pm}-147$} \\
\hline $\begin{array}{l}\text { Norm. Act } \\
(\mathrm{mC} \mathrm{i} / \mathrm{cu}-\mathrm{ft})\end{array}$ & $\begin{array}{l}\text { Cond. Pt. } \\
\text { Probability }\end{array}$ & $\begin{array}{l}\text { Norm. Act. } \\
(\mathrm{mCi} / \mathrm{cu}-\mathrm{ft})\end{array}$ & $\begin{array}{l}\text { Cond. Pt. } \\
\text { Probability }\end{array}$ & $\begin{array}{r}\text { Norm.Act. } \\
(\mathrm{mCi} / \mathrm{cu}-\mathrm{ft})\end{array}$ & $\begin{array}{l}\text { Cond. Pt. } \\
\text { Probability }\end{array}$ \\
\hline $\begin{array}{l}522 . \\
850 .\end{array}$ & $\begin{array}{l}0.5 \\
0.5\end{array}$ & $\begin{array}{l}0.000129 \\
125 . \\
589 .\end{array}$ & $\begin{array}{l}0.98630 \\
0.00685 \\
0.00685\end{array}$ & $\begin{array}{r}507 . \\
1530 . \\
3180 .\end{array}$ & $\begin{array}{l}0.424 \\
0.288 \\
0.288\end{array}$ \\
\hline \multicolumn{6}{|c|}{$P($ Activity $>0)$} \\
\hline & 0.00000437 & & 0.000319 & & 0.00000759 \\
\hline \multicolumn{2}{|c|}{ Unidentified $\beta / \gamma$} & \multicolumn{2}{|c|}{ BET-TRU } & & \\
\hline $\begin{array}{l}\text { Norm. Act. } \\
\text { (mC } \mathrm{i} / \mathrm{cu}-\mathrm{ft})\end{array}$ & ¿ $\begin{array}{c}\text { Cond. Pt. } \\
\text { Probability }\end{array}$ & $\begin{array}{l}\text { Norm. Act. } \\
(\mathrm{mCi} / \mathrm{cu}-\mathrm{ft})\end{array}$ & $\begin{array}{l}\text { Cond. Pt. } \\
\text { Probability }\end{array}$ & & \\
\hline \multirow[t]{3}{*}{$\begin{array}{l}47.6 \\
63.4 \\
209 . \\
313 . \\
319 . \\
731 .\end{array}$} & $\begin{array}{l}0.1762 \\
0.1762 \\
0.1619 \\
0.1619 \\
0.1619 \\
0.1619\end{array}$ & $\begin{array}{c}1.26 \\
3.76 \\
60.6 \\
379 . \\
1090 . \\
2570 .\end{array}$ & $\begin{array}{l}0.7492 \\
0.0394 \\
0.0394 \\
0.0268 \\
0.0268 \\
0.1183\end{array}$ & . & \\
\hline & \multicolumn{3}{|c|}{$P($ Activity $>0)$} & & \\
\hline & 0.0000198 & & 0.0000815 & & \\
\hline
\end{tabular}


Table 11. Overall committed effective dose equivalent from inhalation.

\begin{tabular}{cc}
\hline Normalized C.E.D.E. (Mrem/cu-ft) & $\begin{array}{c}\text { Cond. Cum. } \\
\text { Probability }\end{array}$ \\
\cline { 2 - 2 } 0.0717 & 0.0 \\
0.291 & 0.10 \\
0.97 & 0.20 \\
4.11 & 0.30 \\
5.96 & 0.40 \\
10.2 & 0.50 \\
21.0 & 0.60 \\
37.7 & 0.70 \\
116. & 0.80 \\
360. & 0.90 \\
894. & 0.98 \\
2010. & 0.995 \\
19300. & 0.999 \\
& 1.000 \\
& \\
\hline
\end{tabular}


For example, in Table 4 consider TRU nuclides and $691 \mathrm{mCi} / \mathrm{cu}-\mathrm{ft}$. We have $A=691, P(A)=0.98$, and $p_{\text {pos }}=0.99198$. Therefore, from Equation (1) the probability that a random cubic foot of waste will contain at most $691 \mathrm{mC} i$ of TRU nuclides is

$$
\begin{aligned}
(1- & 0.99198)+0.99198 \times 0.98 \\
& =0.00802+0.97214 \\
& =0.98016
\end{aligned}
$$

Similarly from Table 5, the probability that a random cubic foot of waste will contain at most $1.25 \mathrm{mC} i$ of $\mathrm{Ba}-147 \mathrm{~m}$ is

$$
(1-0.0224)+0.0224 \times 0.30=0.9843 \text {. }
$$

In this example, the dominant contributor to this probability is $1-0.0224$, the probability that the waste will contain no Ba-147m at all.

Eight cases in Tables 4 through 9 involved fewer than twenty shipments: $\mathrm{Cm}$ 144 expressed in $\mathrm{mCi} / \mathrm{cu}-\mathrm{ft}$ and in $\mathrm{mg} / \mathrm{cu}-\mathrm{ft}$, the TRU nuclides from Bettis, and five other individual nuclides listed in Table 5 . Therefore, these cases are also presented in Table 10 by giving the discrete distribution. The conditional probability corresponding to the shipment is the volume of the shipment divided by the total volume of those containers containing the class of interest. The value $p_{\text {pos }}$, shown as $P($ Activity $>0)$, is the total volume of containers containing the class of interest divided by the total volume of all containers in the data set. The unconditional probability that a random cubic foot has one of the normalized activities shown is $p_{\text {pos }}$ times the conditional point probability. The conditional probabilities in Table 10 were found using a hand calculator, and recalculated later as a check. 


\section{How to Use the Tables for Simulations}

The empirical distribution is approximated from the cumulative values in Tables 4-9 and 11. Consider again TRU nuclides as an example. Let $X$ denote the random activity of TRU nuclides in a random cubic foot of waste. It was found above that

$\operatorname{Prob}(X \leq 691)=0.98016$

Similarly, we can find that

$\operatorname{Prob}(X \leq 223)=0.90080$

$\operatorname{Prob}(X \leq 74.9)=0.80160$

and so forth. Therefore, the continuous portion of the distribution of $x$ has a density approximated by a histogram, with

probability $=0.98016-0.90080=0.07936$ between 223 and 691 , probability $=0.90080-0.80160=0.09920$ between 74.9 and 223 ,

and so forth. The entire distribution has a point mass of $1-0.99198=0.00802$ at zero, and above zero it has a density approximated by the histogram on the left side of Table 12. Note that the 13 cell probabilities on the left side of Table 12 sum to 0.99200 , which equals the required 0.99198 except for round off error, and that the sum of these probabilities and the point mass at zero is 1.0 , except for round off error. The "density" times the width of any cell equals the cell probability, as is required of a density. We write it in quotation marks because the total area under the density equals 0.99198 , not the usual 1.0. No graph of the density is shown, because the both the activity and the density range over orders of magnitude; on ordinary axes, most of the graph would vanish into the axes, and on $\log \log$ axes the usual interpretation of the areas would be meaningless.

If the simulation software does not allow a distribution with both a 
Table 12. Histogram of distribution for TRU waste.

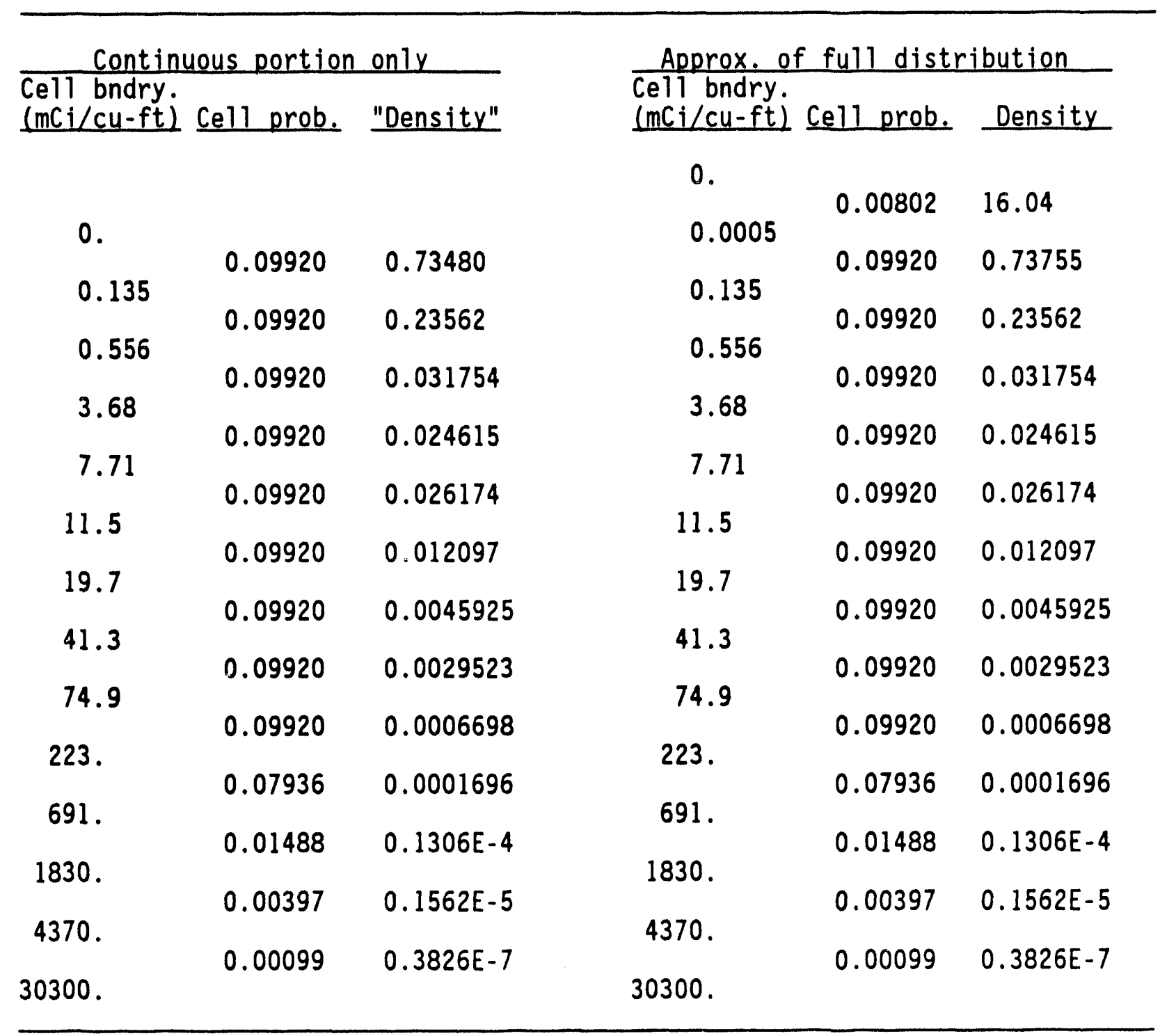


continuous and a discrete portion, approximate the entire distribution by a continuous one: change the point mass at zero to a constant density over a very small range, (very small small compared to the $10 \%$ point), and construct the density so that the area under the curve in that range equals the required point mass. For example, from Table 12, 0.0005 is very small compared to 0.135 . Therefore, let the density be constant in the range 0 . to 0.0005 , with area under the curve there equal to 0.00802 . The value of the density therefore is $0.00802 / 0.0005=16.04$, as shown on the right side of Table 12. The point mass is very small in this example, but it is not small in other examples. Note that the left and right sides of Table 12 are identical except near zero.

Table 13 shows the approximate histogram densities for two other cases: ILTSF non-TRU waste, and committed effective dose equivalent (C.E.D.E) from inhalation. For ILTSF non-TRU waste, the point mass at zero has been approximated by a density over a small range. For the inhalation dose, no such approximation is necessary, because $\operatorname{Prob}($ C.E.D.E. $>0)=1$. These histograms are not shown graphically, for the same reasons that applied to Table 12 . Distributions such as these should be sampled in simulations.

An approximation has been used for th epoint mass at zero. A similar approximation can be used when two cell boundaries are listed as equal. For example, Table 7 shows 389000 both as the $99.9 \%$ point and as the absolute maximum for ILTSF non-TRU waste. To calculate the density in Table 13 we changed these values to 388500 and 389500 (both of which could round off to 389000 ). This made it possible to calculate the density shown in Table 13.

To simulate a random waste container with a volume of $V$ cubic feet, randomly sample the normalized activity $X$ from this histogram distribution, and multiply the selected value of $X$ by the volume $V$, to obtain the activity (mCi) in the simulated container. 
Table 13. Histograms for ILTSF non-TRU waste and inhalation dose.

ILTSF Non-TRU Waste

Cell bndry.

(mCi/cu-ft) Cell prob. "Density"

0 .

0.05
$0.99883 \quad 19.9767$

96.0

0.000117

$0.1219 E-5$

$0.000117 \quad 0.2600 E-5$

141.

161.
0.000117
$0.5850 \mathrm{E}-5$

215.
0.000117
$0.2167 E-5$

249.

$$
0.000117 \quad 0.3441 E-5
$$

302.

495.

858.

2190.

$$
0.000117 \quad 0.2208 E-5
$$

$0.000117 \quad 0.6062 \mathrm{E}-6$

0.000117

$0.3223 E-6$

$$
0.000117
$$

$0.8784 \mathrm{E}-7$

28700 .

$$
0.0000936 \quad 0.3531 E-8
$$

96000 .

388500 .

389500 .

\section{$0.00001755 \quad 0.2608 \mathrm{E}-9$}

$0.00000468 \quad 0.1600 \mathrm{E}-10$

0.00000117

$0.1170 \mathrm{E}-8$
Inhalation C. E. D. E.

Cell bndry.

(Mrem/cu-ft) Cell prob. "Density"

0.

0.0717

0.10

1.3947

0.291

1.97

0.10

0.45600

0.10

0.059559

4.11

0.10

0.046729

5.96

0.10

0.054054

10.2

0.10

0.023585

21.0

37.3

116.

$0.10 \quad 0.0092593$

0.10

0.0059880

0.10

0.0012771

360 .

894.

$0.08 \quad 0.0003279$

$0.015 \quad 0.2809 \mathrm{E}-4$

2010.

19300 .

\section{Cautions}

To simulate an accident scenario involving a breached waste container, one would want to simulate the various nuclides that might be in the container. However, the distributions of the nuclides are not independent: certain nuclides tend to occur together, and there may be pairs of nuclides that tend not to occur together. An obvious example is given by nuclides in a single decay chain. For example, in Table 2 there are 54 shipments containing U-232. As seen in Table 1 , 
U-232 decays to Th-228, which spawns a chain of short-lived isotopes, each isotope quickly decaying to the next. Thus, the same 54 shipments contain U-232, Th-228, Ra-224, Rn-220, $\mathrm{Po}-216, \mathrm{~Pb}-212, \mathrm{Bi}-212, \mathrm{Tl}-208$, and $\mathrm{Po}-212$. From Th-228 to $\mathrm{Bi}-212$, all the elements in the chain have exactly the same activity, because whenever a parent nucleus decays, a daughter is created which then also quickly decays.

Therefore, for typical accident simulations it is desirable to use the high-level classes if possible, rather than simulating the individual nuclides. As an example, suppose we wish to simulate a fire, and are concerned about human exposure through inhalation. One high-level class is the class of $\alpha$-emitters. This class can be simulated directly from the information in Table 4 . It is not so simple, however, to use this simulation to estimate the health effects of the simulated release. From Table 1, the most abundant $\alpha$-emitters are Am-241, Pu238, and $\mathrm{Pu}-239$, and $\mathrm{Pu}-240$, in that order. One could combine their doses by assuming that their proportions in the release are the same as in the entire population, but this ignores the variability in the composition of the waste.

Therefore, the correct way to simulate the health effect is to use the empirical distribution of committed effective dose equivalent. Such a distribution is developed for the inhalation pathway in Table 11, expressed in Mrem $/ \mathrm{cu}-\mathrm{ft}$, where $1 \mathrm{Mrem}=10^{6} \mathrm{rem}$. The unit Mrem may seem surprisingly large, but realize that this corresponds to inhalation of a full cubic foot of waste. In reality, the waste would be dispersed in the air, and someone would inhale only a small fraction of a cubic foot, thereby receiving a small fraction of the tabulated megarems.

Inhalation is regarded as the most important path for managed waste that contains many $\alpha$-emitters. Doses for other pathways could also be developed, but they are not shown here. To compute the committed effective dose equivalent from other paths, modify the programs PREPD.FOR and DOSE.SAS listed in Appendix A. 


\section{CONCLUSIONS}

This report has analyzed radioactive contents of above-ground containers, as 1 isted in the RWMIS data base. The empirical distributions (mCi/cu-ft) have been given: for important classes of nuclides such as the TRU nuclides, the $\alpha$ emitters, the $\beta / \gamma$-emitters, and the fissionable nuclides; for the major individual nuclides; and for classes of containers such as those from a particular waste generator or those in a particular location. The empirical distribution expressed in $\mathrm{mg} / \mathrm{cu}-\mathrm{ft}$ has al so been given for $\mathrm{classes}$ of fissionable nuclides and for the major individual fissionable nuclides. Finally, the empirical distribution of the committed effective dose equivalent has been given for the inhalation pathway, expressed in Mrem $/ \mathrm{cu}-\mathrm{ft}$, where $1 \mathrm{Mrem}=10^{6} \mathrm{rem}$.

To simulate a random container, randomly sample a normalized activity $(\mathrm{mCi} / \mathrm{cu}-\mathrm{ft})$, mass $(\mathrm{mg} / \mathrm{cu}-\mathrm{ft})$, or dose (Mrem/cu-ft) from the tabulated empirical distribution, and multiply this number by the number of cubic feet in the postulated container. This is the simulated activity (mCi), mass (mg), or dose (Mrem) from the container. This can be done for an individual nuclide, some class of nuclides, or some class of containers. 


\section{APPENDIX A: DETAILS OF PROGRAMMING}

\section{dBase Calculations, Including Decayed Activity}

An important part of the dBASE calculations was the calculation of decayed activity, as follows. The half-lives of the most of the radionuclides were taken from the Eleventh Edition of Chart of the Nuclides (cf. Walker et a1., 1984), and had been entered and proof-read for an earlier project (Schlafman, 1992). A few radionuclides had not been encountered in the earlier project. Their half-lives were taken from the Thirteenth Edition (Walker et a1. 1984); they were then double checked to make sure they had been entered correctly.

Generation of daughter radionucl ides is governed by the Bateman equations, found for example in Stephenson (1958). These equations were programmed in dBASE to yield the decayed nuclide inventory. Several simplifications were noted. First, if a daughter nuclide has a very short half-life, a few days or less, then every atom created will quickly decay. Because curies are proportional to disintegrations per second and because every disintegration of the parent results in an almost immediate disintegration of the daughter, the activity in curies of the daughter equals the activity of the parent. Second, in many cases the activity of the daughter was very small, either because there was little of the parent originally present, or because either the parent or the daughter, or both, decayed slowly. We did not generate daughters with activity less than $2 \mathrm{Ci}$. Such cases show the daughter as "negl" in Table 1 . As a result of these two simplifications, only the first daughter's activity ever needed to be calculated from the Bateman equations, and this only needed to be done for six nuclides. The most striking case is Pu-241, which decays to Am-241 and yields more Am-241 than was originally present.

The activity calculations were verified and validated as follows. The dBASE program HC_Decay.prg was written by Schlafman and then examined by Atwood to ensure that the equations had been correctly encoded. Test cases were run and checked by hand calculations; these cases checked both the decay of nuclides and the summing of activities from different inventories. Finally, to check that the 
Bateman equations had been correctly understood and used, the results were compared to results from RadDecay (1987), a public-domain computer program. The results were close, but not exact. Exact agreement cannot be expected because the half-lives in RadDecay are not identical to those used for this project. The above exercise also validated most of H_Decay.prg and C_Decay.prg, described below, because the major portion of all three programs is a call to a single decay procedure.

The only other nontrivial calculation was for shipment volume, because RWMIS reports individual container volumes, often mixing units within a shipment. Test cases were run for the dBASE program that finds the total volume of each shipment. The results agreed with hand calculations.

\section{dBASE Programs}

The following progranis were used to calculate original and decayed activities and write the data base file information into ASCII text files which were used by the Fortran and SAS programs. The programs were written using the Foxpro 2.0 software. Since no Foxpro-specific commands were used in these programs, the programs could be compiled and run in dBASEIV software.

Verify.prg - The program calculates the totals of all numeric fields in RWMIS download files, checks for matching shipment identifiers in the download files, and calculates record counts for the files. The information is then compared to the values calculated in RWMIS to ensure the data was downloaded correctly.

HC_Decay.prg - The program calculates the original and decayed activities for each nuclide for all shipments in the Historical and Current RWMIS data. It uses information in the Container and Nuclide data files and in the file HC_Nuc.dbf, all described below. It replaces the original and decayed activities in $\mathrm{HC}$ Nuc.dbf, and writes the information to HC_Decay.txt, which was imported electronically to produce Table 1 of this report. 
HC_Decay.prg was run first with no generation of daughters. Then each nuclide was individually decayed for the average time described below, using the public domain program RadDecay, to see if more than $2 \mathrm{Ci}$ of a daughter would be generated. If it would, the decay equations for the nuclide were added to HC_Decay.prg, H_Decay.prg, and C_Decay.prg. At the end of this investigation, $H_{C}$ Decay.prg was rerun to produce a final version of HC_Decay.txt for Table 1.

Ave_time - This takes the original and decayed total activity in HC_Nuc.dbf, (based on many shipments on many dates) and finds a single time span which would have produced the same decay. It enters this "average time" in HC_Nuc.dbf.

This average time was used in simple calculations to determine, for each nuclide, if more than $2 \mathrm{Ci}$ of the daughter would be generated. If it would, the equations for generation of that daughter were programmed into HC_Decay.prg, H_Decay.prg, and C_Decay.prg.

H_Decay.prg - The program calculates the decayed activities for the Historical RWMIS data for each individual shipment. It uses the data files HistNuc.dbf and HistCont.dbf, and writes the decayed activities and the other RWMIS information required to H_Decay.txt. This text file containing the individual shipment information is read by the Fortran programs. For each shipment, it modifies the curies in HC_Nuc.dbf.

C_Decay.prg - The program calculates the decayed activities for the Current RWMIS data for each individual shipment and writes this information and the other RWMIS information required to C_Decay.txt. The individual shipment information is then read by the Fortran programs. Note: The only difference between C_Decay.prg and H_Decay.prg is the RWMIS data file and field names. 


\section{RWMIS Download Data Files}

Each RWMIS download consists of three files: the Master file, Container file, and Nuclide file. The Master file contains shipment total information such as total shipment curies. No data from this file was used in this study. The Container file contains information by groupings of containers within shipments. The Nuclide file contains information about the individual nuclides in each shipment. Each RWMIS download file contains six key fields which are used to identify unique shipments and relate the information between files. The key fields are generating area, waste type, radioactivity, on/off site, report date, and report time.

CurMstr.dbf

HistMstr.dbf - Only used in verification of RWMIS download.

CurNuc.dbf

HistNuc.dbf - The RWMIS download data base file of the nuclide information. Information used from this file consists of the key fields, nuclide identifier, and nuclide curie amount.

CurCont.dbf

HistCont.dbf - The RWMIS download data base file of the container information. Information used from this file consists of the key fields, disposal location, container volume, volume units, and number of containers.

Other Data Base Files

HC_Nuc.dbf - The data base file contains the nuclide identifier, half-life, original curies, decayed curies, transuranic information, $\alpha$-emitter information, $\beta / \gamma$-emitter information, fissionable information, daughter product, and average time. Immediately after the file has been modified by HC_Decay.prg, the information is used to produce Table 1. The file is also 
modified by Ave_time.prg, H_Decay.prg, and C_Decay.prg. An additional field for the inhalation dose conversion factor was not used for this study but has been added for possible future studies.

Hold.dbf - $\quad$ The data base file is used in the H_Decay.prg and C_Decay.prg to hold the nuclide identifier, report date, and nuclide curie amount for individual shipments. This file is a temporary file which holds one shipment's information to be used in the decay calculation. The file is cleared and populated for each shipment.

For future studies the following should be considered. The download request could include only the fields and files that are used, which would save disk space. The RWMIS totals of the numeric fields and the number of records downloaded should be requested in order to verify the download. Verify.prg was created to verify a download with all three RWMIS files with all of the fields. Minor modifications to the Verify.prg would be required to verify a subset of the data. The RWMIS download field names should be checked to ensure that they match the fields hardcoded in the programs.

Any nuclide identifiers that were not used in this study, but are required for the next study, will need to be entered in the HC_Nuc.dbf with the related information about the nuclide. After the original and decayed cur:es had been calculated, Ave_time.prg calculated the average decay time and $p l$ aced this number in the nuclide average time field in $\mathrm{HC}_{-}$Nuc.dbf. The average time and original decay were then entered into the RadDecay application to obtain information about which nuclides would have daughter products with activity greater then 2 curies. In the future, this process would only be required to find the daughter products for nuclides that were not in the present study or that had larye increases in activities. Finally, for this study the inhalation dose conversion factors were added manually to a text file. For possible future studies they have been added to the data base HC_NuC.dbf.

The dBASE programs are given here. 


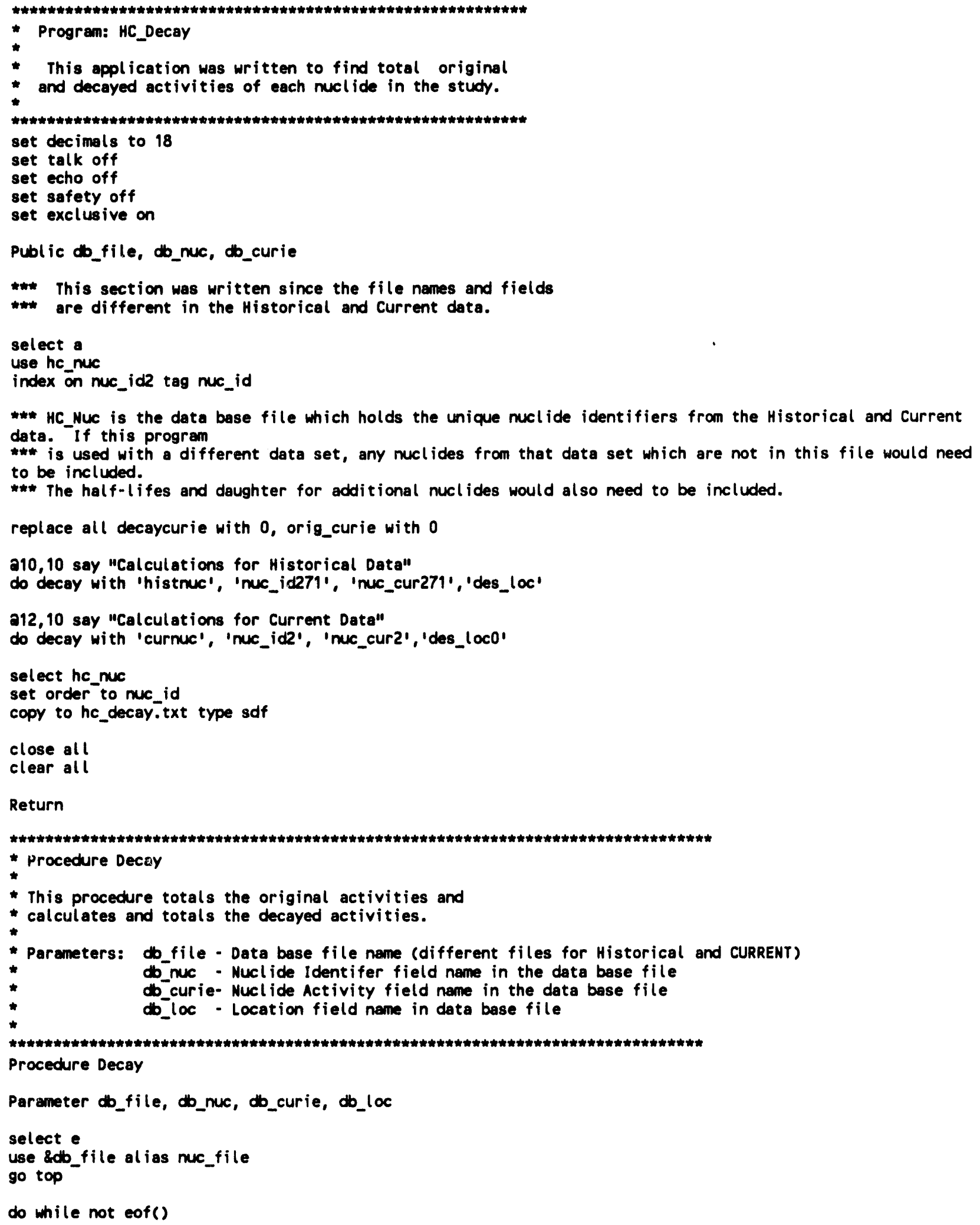




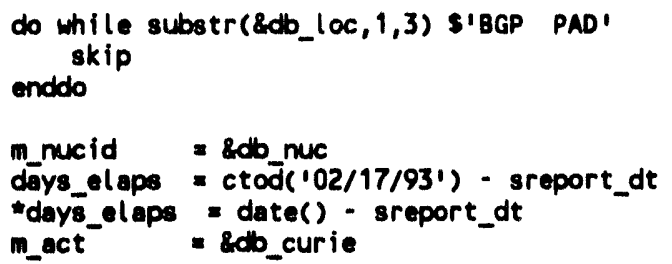


enddo

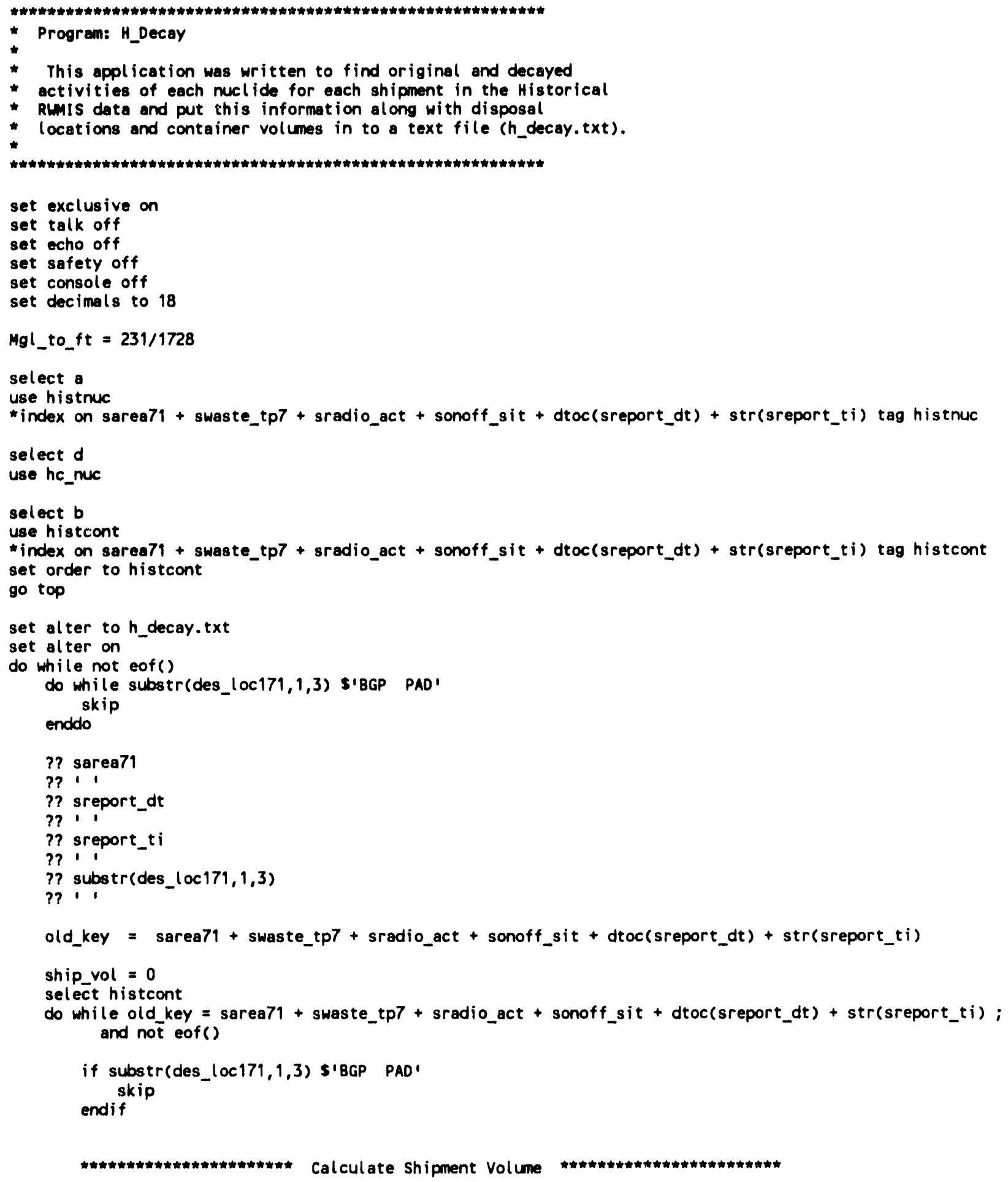




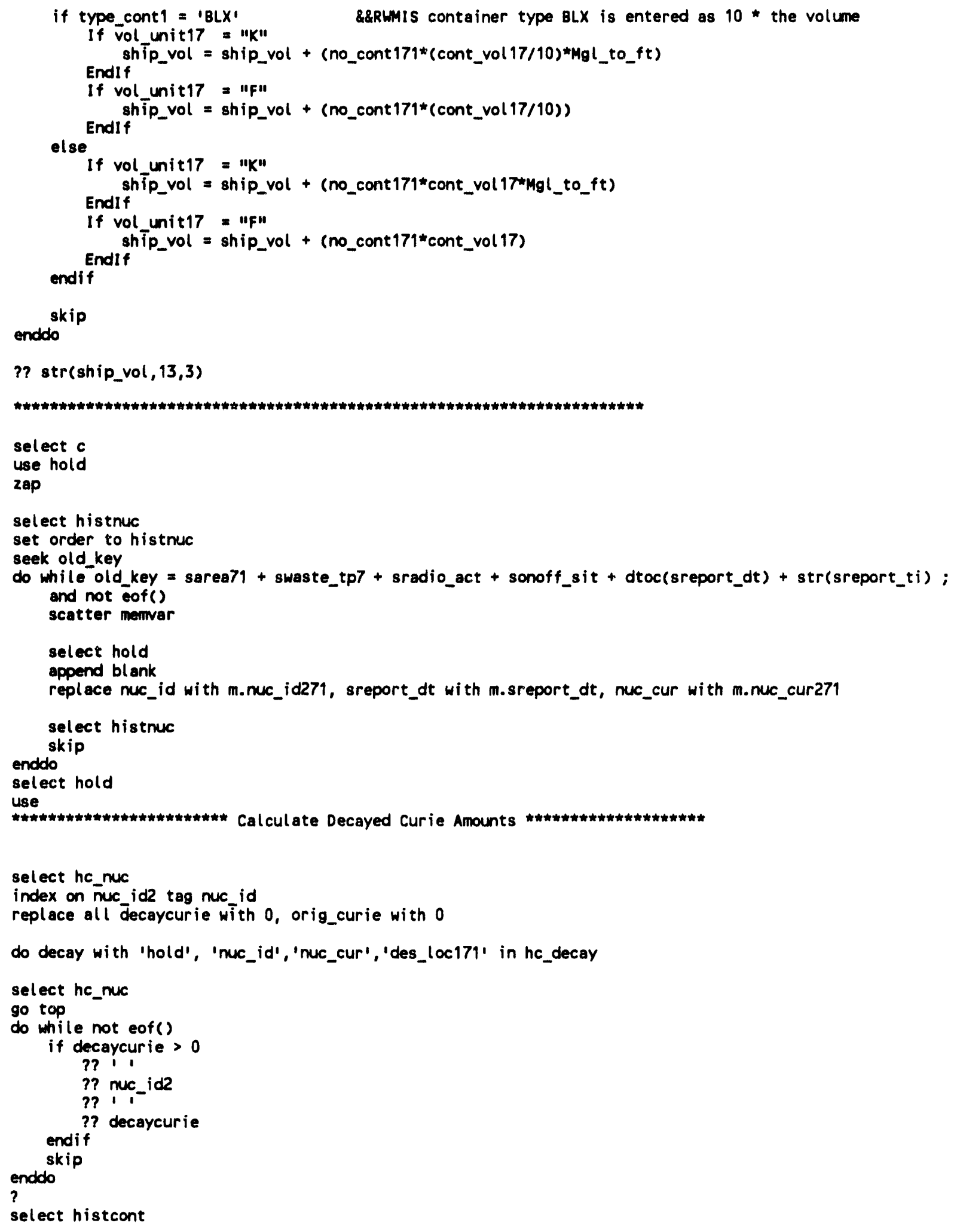




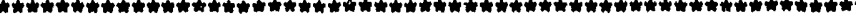

- Programa C_Decay

- This application was written to find original and decayed

- activities of each nuclide for each shipment in the Current

* RLMIS data and put this information along with disposal

* locations and container volumes in to a text file (C_decay.txt).

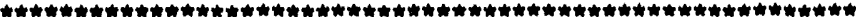

set exclusive on

set talk off

set echo off

set safety of $f$

set console off

set decimals to 18

Mgl_to_ft $=231 / 1728$

select a

use curnuc

*index on sarea + swaste tp + sradio act + sonoff sit + dtoc(sreport dt) + str (sreport ti) tag curnuc

-index on sarea71 + swaste_tp7 + sradio_act + sonöf_sit + dtoc(srepörtdt) + str(sreport_ti) tag curnuc

select $b$

use curcont

*index on sarea + swaste_tp + sradio_act + sonoff_sit + dtoc(sreport_dt) + str(sreport_ti) tag curcont

select $d$

use hc_nuc

select curcont

set order to curcont

go top

set alter to c_decay.txt

set alter on

do while not eof()

do while substr(des_loc1,1,3) \$'BGP PAD'

skip

enddo

?? sarea

?? 1

?? sreport_dt

?? 11

?? sreport ti

?? 11

$?$ ? substr(des_loc $1,1,3$ )

?? I '

old_key = sarea + swaste_tp + sradio_act + sonoff_sit + dtoc(sreport_dt) + str(sreport_ti)

ship_vol $=0$

select curcont

do while old_key $=$ sarea + swaste_tp + sradio_act + sonoff_sit + dtoc(sreport_dt) + str(sreport_ti) and not eof()

if substr(des $\operatorname{loc} 1,1,3)$ S'BGP $P A D$

$$
\text { skip }
$$

endif 


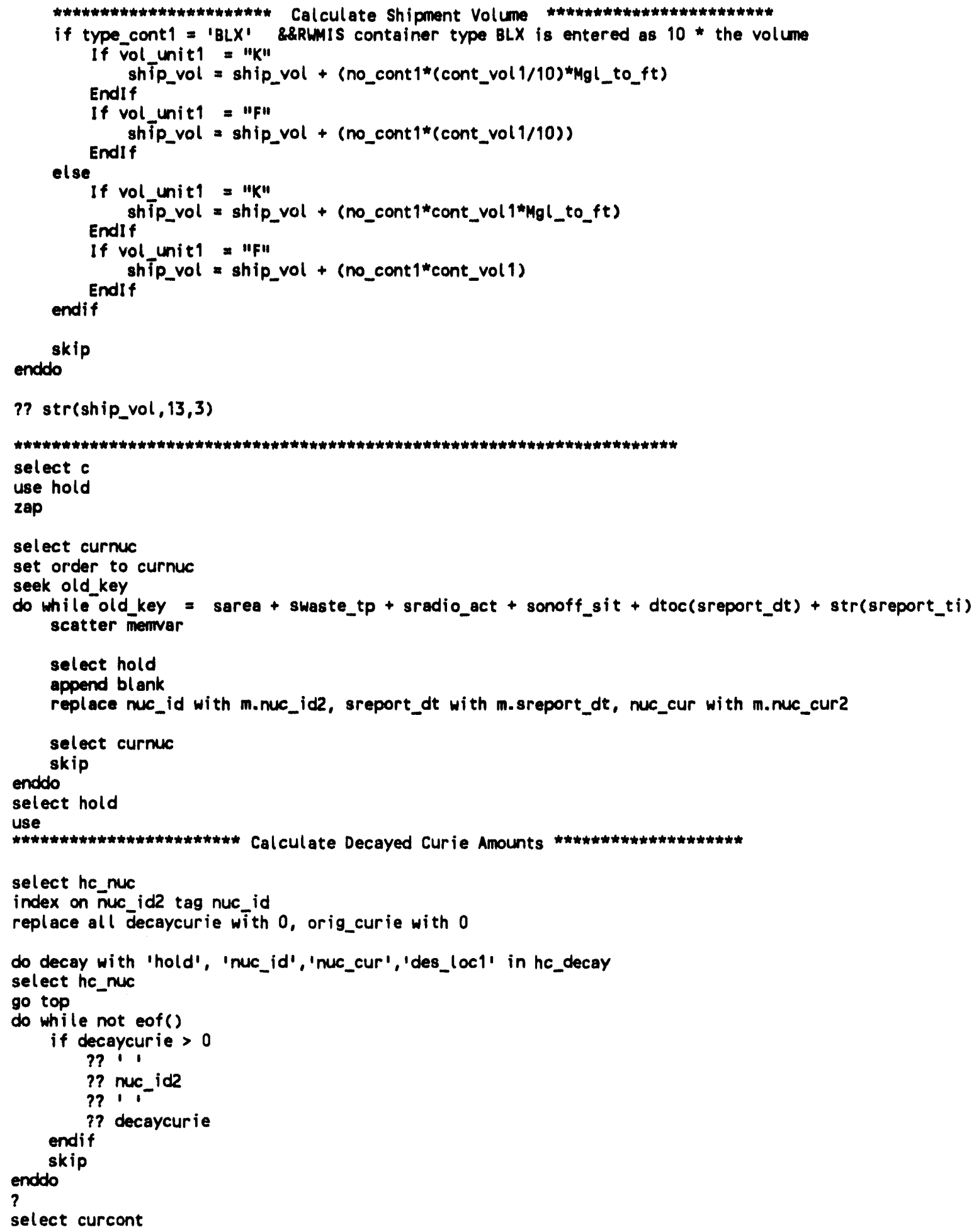




\section{Fortran Programs to Summarize Data and Get Normalized Activities}

Three programs were used to read the output of the dBASE programs and prepare files for SAS to analyze. One program, PREP, wrote files for classes of nuclides, such as TRU nuclides, $\alpha$-emitters, and fissionable nuclides. The second, PREPI, wrote files for the individual nuclides. The third, PREPD, wrote inhalation doses for the shipments. The programs were compiled using the Lahey (1992) Fortran compiler, but they are written in ANSI standard Fortran, except for the use of lower case for debug lines, and so should run with any modern compiler. The only problem might be with the number of files simultaneously open.

Other short Fortran programs were used: TALLY summarizes the data, as presented in Table 2. PNZERO calculates the probability that a random cubic foot would contain non-zero activity of the class of interest; these probabilities are shown in Tables 4 through 11.

Two inputs for the programs were H_Decay.txt and C_Decay.txt, created by the dBASE programs. A third input for the program is nucinf.dat, a file modified from HC_Decay.txt, which was created by the dBASE program HC_Decay.prg. HC_Decay.txt was turned into nucinf.dat by electronically moving columns, and by manually adding the inhalation dose conversion factors. These conversion factors (rem/ $\mu \mathrm{C} i$ ), give the committed effective dose equivalent (C.E.D.E.). They are taken from DOE/EH-0071 (1988). That report lists factors for all the nuclides in the decayed RWMIS inventory except for Ba-137m, Kr, the Po isotopes 212 through 218, Rh-106, Rn, and T1-208. These isotopes have only small activities in the decayed RWMIS data set. The factors were copied to the file nucinf.dat by John Poloski and C. Atwood. When there were several factors, corresponding to different possible retention periods or chemical forms, the maximum listed value was used. Atwood then proofread the file nucinf.dat, finding and correcting four errors. Only the high-activity isotopes are important for this study; including factors for all the isotopes does not change Table 11 at all, but could be important if the inventory were different. The file nucinf.dat is shown below as Table Al. 
PREP, PREPI, PREPD, and TALLY were all run first using only the first ten shipments of the Historic Data and the first ten shipments of the Current Data. The output, including intermediate output printed for debugging purposes, was examined, and was found to be correct: the nuclides in the data bases were tallied, the $\mathrm{mci} / \mathrm{cu}-\mathrm{ft}$ and (for fissionable nuclides) $\mathrm{mg} / \mathrm{Cu}-\mathrm{ft}$ and (for shipments) rem/cu-ft were calculated correctly, and everything was summed correctly and written to the proper files. Then the programs were run with the debug print statements turned off, and using the entire data base. The file for $Y-90$ (the last of the nuclides with high enough activity to be treated individually) was examined; it was seen that the first and last instances of $Y-90$ in both the Historic and Current data sets were correctly written to the Y-90 file. The output from PNZERO was checked in a few cases and found to be correct; this program only computes ratios of volumes, and so is easy to check.

The output file from TALLY was edited slightly with a text editor, and transferred electronically into Table 2 of this report. The output from PNZERO was entered manually into Tables 4 through 10, and every entry was checked on a later day. Out of 78 entries, 2 errors were found and corrected.

Table Al and the Fortran programs are listed here.

Table Al. The file nucinf.dat

\begin{tabular}{|c|c|c|c|c|c|}
\hline $\begin{array}{l}A C-227 \\
A M-241 \\
A M-242 M \\
A M-243 \\
B A-137 \\
B 1-212 \\
81-214 \\
B K-249 \\
C-14 \\
C E-144 \\
C F-249 \\
C F-252 \\
C M-242 \\
C M-243 \\
C M-244 \\
C M-246 \\
C M-248 \\
C O-58 \\
C O-60 \\
C R-51 \\
C S-134 \\
C S-137 \\
E U-150\end{array}$ & $\begin{array}{l}7.947 E+03 \\
1.580 E+05 \\
5.146 E+04 \\
2.690 E+06 \\
1.771 E-03 \\
4.205 E-02 \\
1.375 E-02 \\
3.200 E+02 \\
2.091 E+06 \\
2.850 E+02 \\
1.281 E+05 \\
9.636 E+02 \\
1.630 E+02 \\
1.040 E+04 \\
6.606 E+03 \\
1.744 E+06 \\
1.241 E+08 \\
7.090 E+01 \\
1.930 E+03 \\
2.770 E+01 \\
7.540 E+02 \\
1.100 E+04 \\
5.250 E-01\end{array}$ & 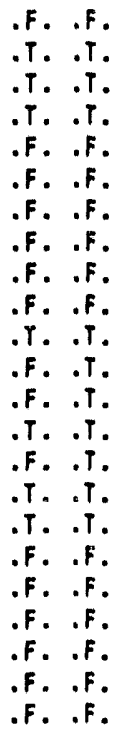 & 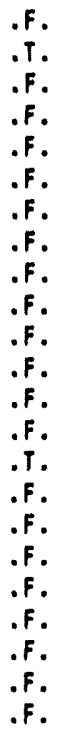 & $\begin{array}{r}0.04079081028 \\
89781.13529897256 \\
0.00030014875 \\
0.37986297170 \\
2245.77511697818 \\
26.55180072599 \\
0.00000000000 \\
0.00057094575 \\
0.00004224624 \\
27.06352985230 \\
0.00972888594 \\
0.00407666951 \\
0.00000143566 \\
0.01530852458 \\
538.99248475109 \\
0.00152990262 \\
0.00000004170 \\
0.00000011008 \\
94.30234207016 \\
0.00000000000 \\
110.74067620797 \\
2003.00961321957 \\
0.00000000000\end{array}$ & $\begin{array}{l}6.7 e+3 \\
5.2 e+2 \\
5.1 e+2 \\
5.2 e+2 \\
0.1 \\
2.1 e-2 \\
6.3 e-3 \\
1.3 e 00 \\
2.1 e-3 \\
3.5 e-1 \\
5 \cdot 5 e+2 \\
1.3 e+2 \\
1.7 e+1 \\
3.5 e+2 \\
2.7 e+2 \\
5.4 e+2 \\
1.9 e+3 \\
7.1 e-3 \\
1.5 e-1 \\
2.6 e-4 \\
4.7 e-2 \\
3.2 e-2 \\
2.7 e-1\end{array}$ \\
\hline
\end{tabular}




\begin{tabular}{|c|c|c|c|c|}
\hline $\begin{array}{l}E U-152 \\
E U-154 \\
E U-155 \\
P E-55 \\
P E-59 \\
H-3 \\
\text { KR-85 } \\
M A P \\
\text { MFP } \\
M N-54 \\
N B-95 \\
N I-63 \\
N P-237 \\
P A-233 \\
P B-210 \\
P B-212 \\
P B-214 \\
P H-147 \\
P O-212 \\
P O-214 \\
P O-216 \\
P O-218 \\
P R-144 \\
P U-236 \\
P U-238 \\
P U-239 \\
P U-240 \\
P U-241 \\
P U-242 \\
R A-224 \\
R A-226 \\
R A-228 \\
R H-106 \\
R H-220 \\
R N-222 \\
R U-106 \\
S B-125 \\
S R-90 \\
T A-182 \\
T C-99 \\
T H-228 \\
T H-230 \\
T H-232 \\
T H-234 \\
T L-208 \\
U-228 \\
U-232 \\
U-233 \\
U-234 \\
U-235 \\
U-236 \\
U-238 \\
U N-1 D-B+G \\
Y-90 \\
Z N-65 \\
2 R-95 \\
2 R \\
\end{array}$ & $\begin{array}{l}4.928 E+03 \\
3.135 E+03 \\
1.719 E+03 \\
9.970 E+02 \\
4.450 E+01 \\
4.490 E+03 \\
3.906 E+03 \\
1.930 E+03 \\
1.100 E+04 \\
3.120 E+02 \\
3.500 E+01 \\
3.650 E+04 \\
7.811 E+08 \\
2.700 E+01 \\
8.140 E+03 \\
4.433 E-01 \\
1.861 E-02 \\
9.563 E+02 \\
4.6065 E-12 \\
1.895 E-09 \\
1.736 E-06 \\
2.160 E-03 \\
1.200 E+00 \\
1.040 E+03 \\
3.201 E+04 \\
8.796 E+06 \\
2.394 E+06 \\
5.256 E+03 \\
1.369 E+08 \\
3.660 E+00 \\
5.840 E+05 \\
2.102 E+03 \\
3.461 E-04 \\
6.435 E-04 \\
3.820 E+00 \\
3.723 E+02 \\
1.007 E+03 \\
1.062 E+04 \\
1.140 E+02 \\
7.774 E+07 \\
6.982 E+02 \\
2.752 E+07 \\
5.110 E+12 \\
2.410 E+01 \\
2.120 E-03 \\
6.319 E-03 \\
2.555 E+04 \\
5.840 E+07 \\
8.979 E+07 \\
2.570 E+11 \\
8.541 E+09 \\
1.632 E+12 \\
1.100 E+04 \\
2.670 E+00 \\
2.440 E+02 \\
6.400 E+01\end{array}$ & 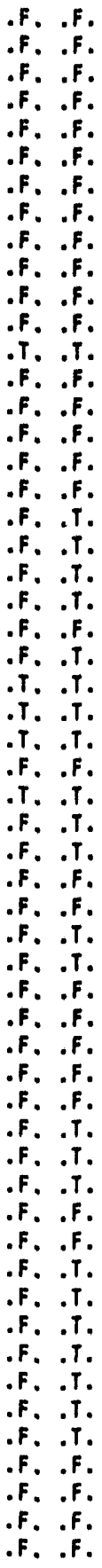 & 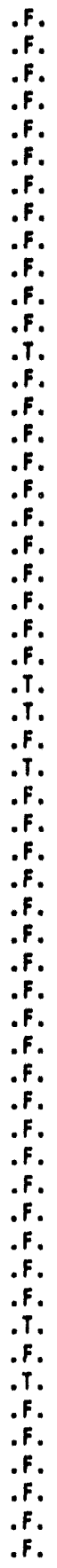 & $\begin{array}{r}0.06756042914 \\
0.71041973487 \\
0.18629208191 \\
1.13167058750 \\
0.0000000000 \\
0.79779881007 \\
6.85660232651 \\
1.28001878301 \\
483.32117589191 \\
0.22870126168 \\
0.00000000000 \\
3.57242150533 \\
0.35519815316 \\
0.00000000000 \\
0.01755985982 \\
26.55180072599 \\
0.00000000000 \\
27.25851802530 \\
17.01173872514 \\
0.0000000000 \\
26.55180072599 \\
0.00000000000 \\
27.17796338233 \\
0.01478722382 \\
60802.74635717693 \\
40146.41832407012 \\
9825.29565579516 \\
161122.35948397170 \\
0.94505422615 \\
26.55180072599 \\
0.04789760836 \\
0.03061790908 \\
0.00000000000 \\
26.55180072599 \\
0.00000000000 \\
0.18652829809 \\
1.64659464414 \\
1777.53891178629 \\
0.00001547961 \\
0.00173990688 \\
26.55180072599 \\
0.01998005181 \\
0.33011775515 \\
0.00000000000 \\
9.54006200085 \\
0.00000000000 \\
26.04479556052 \\
898.50468890707 \\
5.77791004370 \\
0.06668774161 \\
0.00119942767 \\
0.11706389623 \\
12.44449056014 \\
2018.60244723459 \\
0.00000001868 \\
0.00000000234\end{array}$ \\
\hline
\end{tabular}

PROGRAM PREP

Reads text-file from dBASE massage of RLMIS download, and normalizes by cubic foot 
C*thardwired NNUCS, KLOC, MBOR, NGRP

PARAMETER (NGRP $=5$, NBMAX $=45$, NTHREE $=3$,

$+\quad$ KLOC $=2$, NORMAX $=30$, NNUCS $=79$, NBOR=7)

CHARACTER\#3 LOC, LOCA(KLOC), OR, ORA (NORMAX), BORA(NBOR)

CHARACTER*6 PART

CHARACTER 13 MUCA(NNUCS), NUCT(NBMAX), NUCGRP(NGRP),

$+\quad$ MUCF IS (NTHREE), NUCBLK

DOUBLE PRECISION CIPF(NNUCS), CIT(NBMAX), GPF(NNUCS),

$+\quad$ CITOT, CITRU, CINOT, CIÁl, CIBEg,

$+\quad$ GTOT, GTRU, GNOT, CITOTS(NGRP),

$+\quad$ GTOTS(NTHREE)

EQUIVALENCE (CITOTS(1),CITOT),(CITOTS(2), CITRU),(CITOTS(3), CINOT),

$+\quad$ (CITOTS(4),CIALF),(CITOTS(5), CIBEG), (GTOTS(1), GTOT),

$+\quad$ (GTOTS(2),GTRU), (GTOTS(3),GNOT)

c CITOTs array is equivalent to (CITOT, CITRU, CINOT, CIALF, CIBEG)

c GTOTS array is equivalent to (GTOT, GTRU, GNOT)

DIMENSION TOTACT(NNUCS), ATHO(NNUCS),

$+\quad$ HaLf(NNUCS), IORM(NORMaX)

LOGICAL TRU(NNUCS), ALF(NNUCS), FIS(NNUCS), BIGACT

logical debug

C $\quad$ CIPF $=m C i / C U-f t, A C T=C i$

DATA NUCGRP/' All',' TRU',' non-TRU',' Alpha',' Beta-gamma'/

DATA NUCFIS/' All Fis', TRÚ Fis', ' nON-TRU Fis'/

DATA LOCA/'TSA', 'ILT'/

DATA BORA/'ALE', 'ANL', 'BET', 'CPPI, 'MRC', 'RFO', 'WMC'/

DATA NUCBLK/'

'\%

DATA IGBASE/6/, IFBASE/11/,ILBASE/14/, 1OBASE/16/

C Anyone who modifies this program should then run it with

debug=. true. before trying it with debug=. false.

Setting debug=.true. limits the analys is to 20 shipments, and generates lots of printout for those shipments.

data debug/. false.l

C Statement function defining "big" activities $\operatorname{BIGACT}(N)=\operatorname{TOTACT}(N)$.GT. 2 .

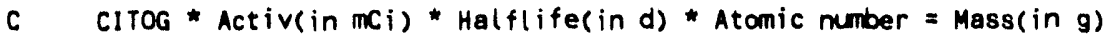
CITOG $=(2.798 \mathrm{E}-9) / 365.24$

OPEN (UNIT=6, FILE=' mdebug. Prp')

OPEN (UNIT $=4, F I L E=$ ' nucinf. dat')

DO $20 \mathrm{~N}=1$, NNUCS

Get nuclide name, half-life (d), properties and total activity

C The file 4 is a massaged version of Table 1 in Atwood \& Schlafman

C If health effects were to be included, this would be a good place

C to input the health effect of each nuclide, as a multiplier of

C activity in curies.

$\operatorname{READ}(4, *) \operatorname{NUCA}(N), \operatorname{HALF}(N), \operatorname{TRU}(N), \operatorname{ALF}(N), \operatorname{FiS}(N)$,

+ TOTACT(N)

C Get atomic numbers from the nuclide names

c if(debug) write $(6,1(1$, nuca $(n)=11, a) 1)$ nuca(n) READ (HUCA (N), ' (A6)' ') PART

C if(debug) write $(6,1(1$, part $=11, a) 1)$ part

IF(PART.EQ. 'LES-TH') THEN

Arbitrary atomic number ATNO(N) $=100$

ELSE IF (PART.EQ.'MAP 1) THEN

C Treat as Co-60 


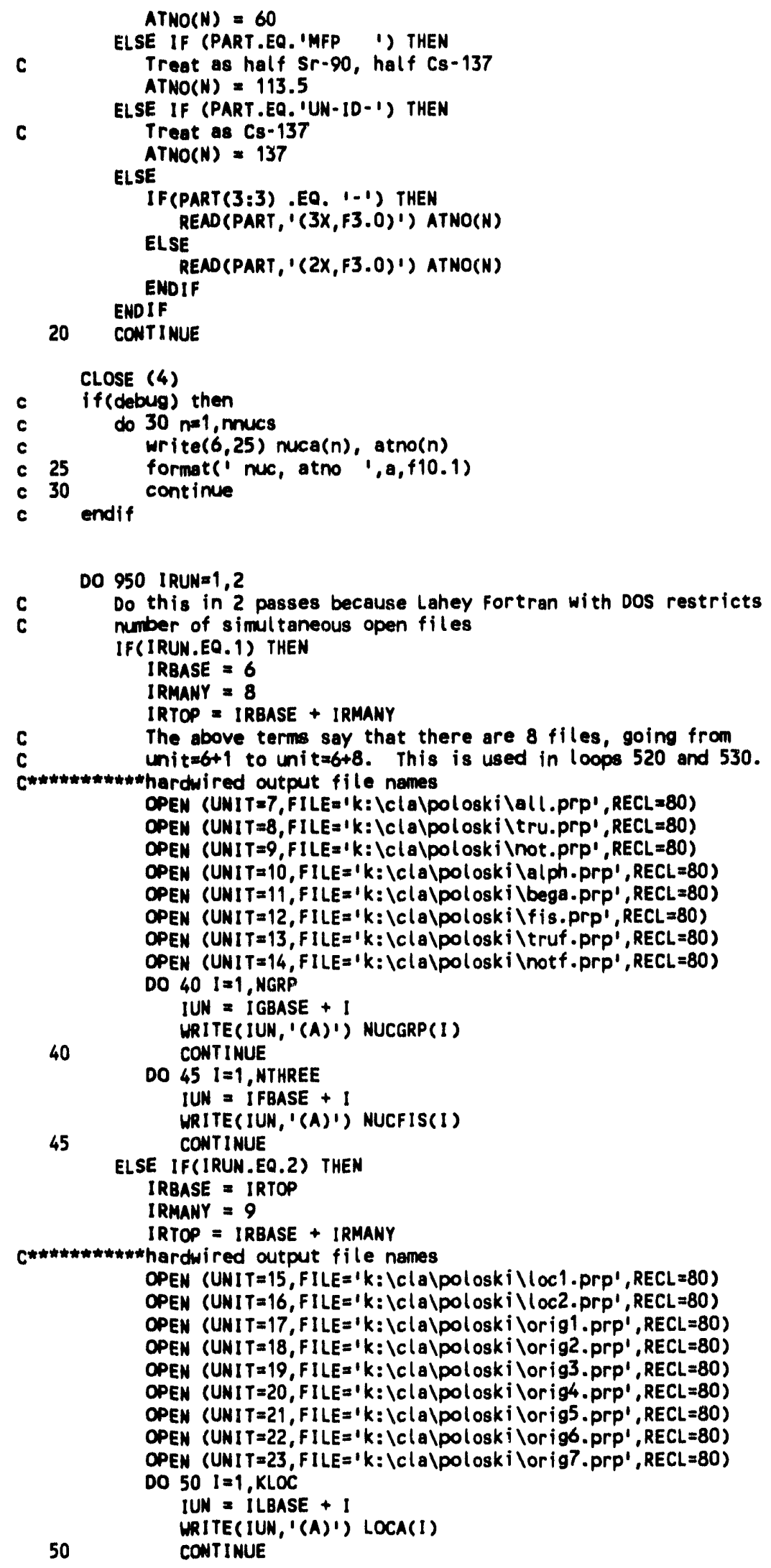




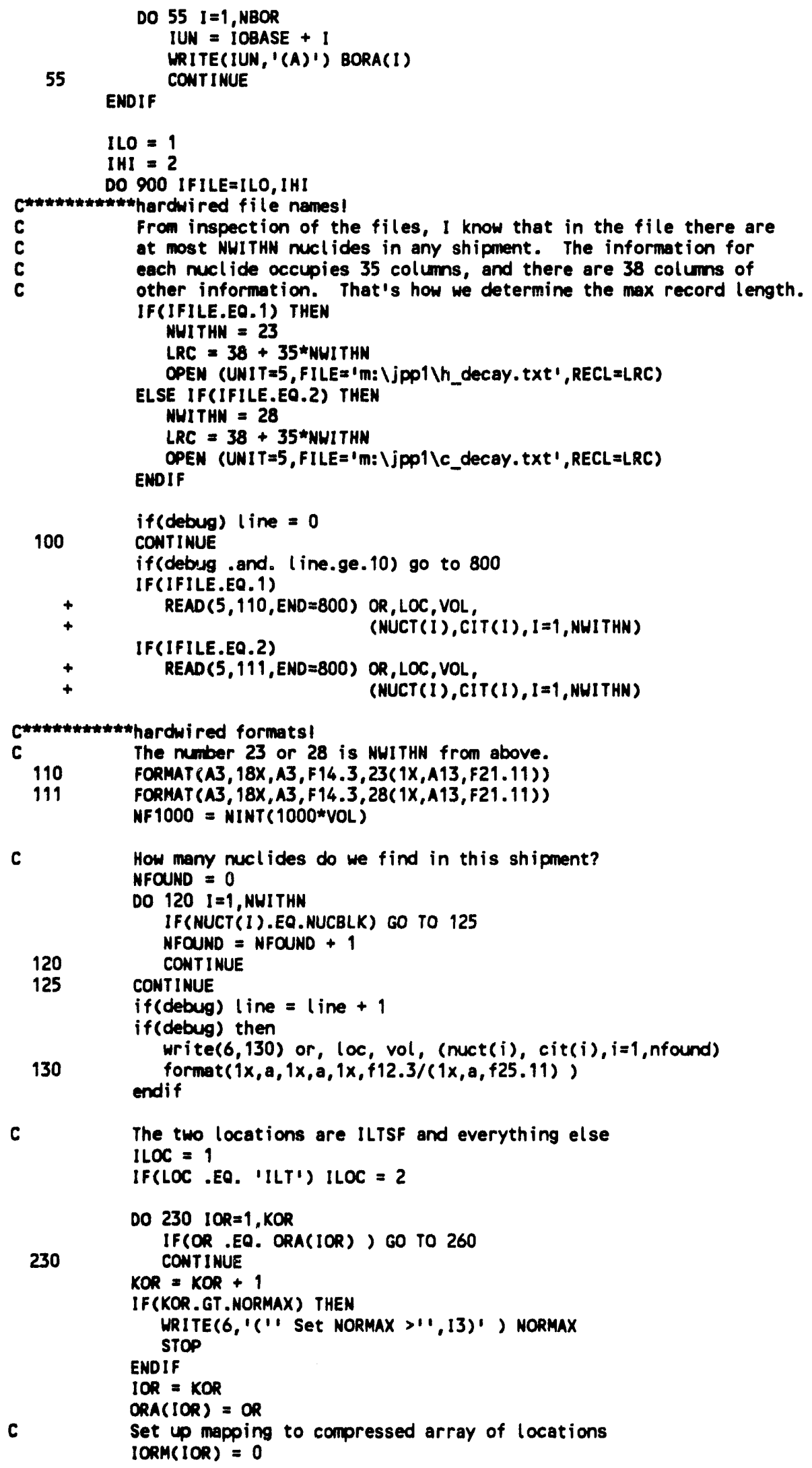


240

DO $240 \quad I=1$, NBOR

IF(BORA(I). NE.OR) 60 TO 240 IORM(IOR) $=1$

co To 260

CONTINUE

CONTINUE

if(debug) write(6, '("' above loop 3201')')

DO $320 \mathrm{N=1}$, NNUCS

$\operatorname{CIPF}(N)=0$

$\operatorname{GPF}(N)=0$

CONTINUE

CITOT $=0$

If heal th effects are to be included, initialize them to zero

in loop-320, and calculate them in loop 360 .

DO $360 \quad 1=1$, NFOUND

CITOT = CITOT + CIT(I)

DO $350 \quad N=1$, NNUCS

IF(NUCT(I) . ME. MUCA(N)) GO TO 350

Successfully read nuclide name in data

CIPF is $\mathrm{mCi} / \mathrm{cu}-\mathrm{ft}$, and GPF is $\mathrm{g} / \mathrm{cu}-\mathrm{ft}$.

$\operatorname{CIPF}(N)=1000 * \operatorname{CIT}(I) / \mathrm{VOL}$

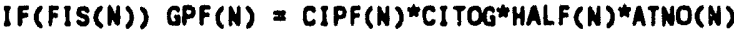

GO TO 360

CONTINUE

MRITE $(6,355)$ MUCT(1), OR, LOC

FORMAT(' Error: ', $A$, ' not found. $I, A)$

CONTINUE

CITOT $=1000^{*} \mathrm{CITOT} / \mathrm{VOL}$

IF(CITOT.EO.0) GO TO 100

SUn activities for TRU, non-TRU, alpha, beta-gamma,

by loc, by orig, and fissile

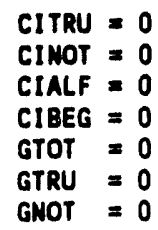

$\mathbf{T}=0$

Recall CIPF(N) is mCi/Cu-ft in the current shipment, for Wth nuclide in list of all nuclides in RLwis

GPF is similar, but $\mathrm{g} / \mathrm{cu}-\mathrm{ft}$

if(debug) write(6,' ('' above loop 500'')')

DO $500 \mathrm{~N}=1$, NNUCS

IF(TRUCN)) THEN

CITRU $=$ CITRU $+\operatorname{CIPF}(N)$

ELSE

CINOT $=$ CINOT + CIPF $(N)$

ENDIF

IF(ALF(N)) THEN

CIALF = CIALF + CIPF $(N)$

ELSE

CIBEG $=$ CIBEG $+\operatorname{CIPF}(N)$

ENDIF

IF(FIS(N)) THEN

GTOT = GTOT + GPF(N)

IF(TRU(N)) THEN

GTRU = GTRU + GPF(N)

ELSE

ENDIF

ENDIF

CONTIMUE

if (debug) then 


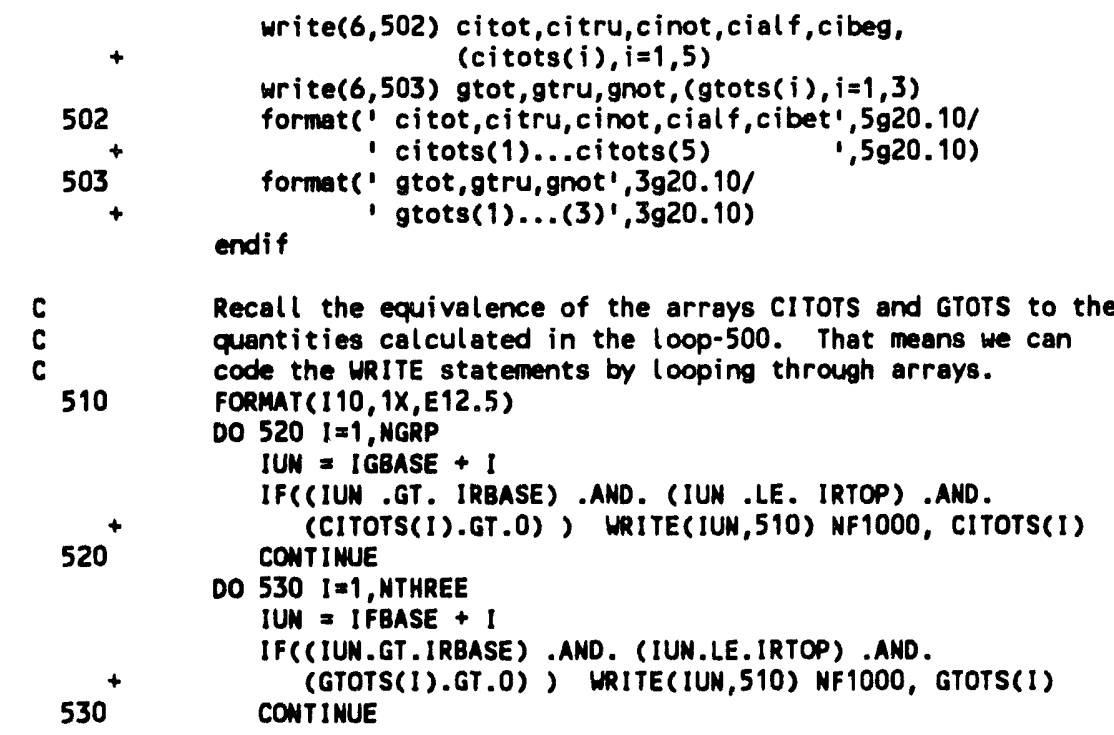

C For consistency with the other files, I suppose I should have written a separate file for each location or origin total, or TRU total, or non-TRU total ( $7 \times 3=21$ files). Instead, I wrote 7 files with 3 columns each, and used missing values (coded as 1.1) for values that are zero. IUN = ILBASE+ILOC

IF(IUN.GT. IRBASE .AND. IUN.LE.IRTOP) THEN

IF(CITRU.GT.O .AND. CINOT.GT.0) THEN WRITE(IUN, 540) NF1000, CITOT, CITRU, CINOT

ELSE IF(CITRU.GT.0) THEN

WRITE(IUN, 545) NF1000, CITOT, CITRU

ELSE IF(CINOT GT 0) THEN

WRITE(IUN,550) NF1000, CITOT, CINOT

ENDIF ENDIF

IF(IORM(IOR).NE.0) THEN

IUN = IOBASE + IORM(IOR)

IF(IUN.GT.IRBASE .AND. IUN.LE.IRTOP) THEN

IF(CITRU.GT.O .AND. CINOT.GT.0) THEN HRITE(IUN, 540) NF1000, CITOT, CITRU, CINOT ELSE IF(CITRU.GT.0) THEN HRITE(IUN, 545) NF1000, CITOT, CITRU ELSE IF(CINOT.GT.O) THEN WRITE(IUN, 550) NF1000, CITOT, CINOT ENDIF

ENDIF

540 FORMAT $(110,3(1 X, E 12.5))$

$545 \quad$ FORMAT $(110,2(1 X, E 12.5), 7 X, 1,1)$

550 FORMAT $(110,1 X, E 12.5,7 X, 1,1,6 X, E 12.5)$

6O TO 100

$\begin{array}{cl}800 & \begin{array}{l}\text { CONTINUE } \\ \text { End of input file. } \\ \text { Cl.OSE (5) } \\ \text { CONTINUE }\end{array} \\ 900 & \text { CONIN }\end{array}$

$$
\begin{aligned}
& 910 \text { DO } 910 \quad I=1 \text {, IRMANY } \\
& \text { CLOSE (IRB, SE + I) } \\
& 950 \text { CONTINUE } \\
& \text { CLOSE (6) } \\
& \text { END }
\end{aligned}
$$


PROGRAM PREPI

Reads text-file from caASE massage of RumIS download, and normalizes by cubic foot

Writes individual isotopes to files of different lengths, for SAS

This is a modification of PREP. See comments there.

WRITTEN BY C. ATHOCO, FEb 1993

IMPLICIT REAL(A-H, O-Z), INTEGER(I-N)

C****thardwired NNUCS

PARAMETER(NBMAX $=45$, NFMAX $=10$, NMUCS $=79$ )

CHARACTER 3 LOC, OR

CHARACTER 6 PART

CHARACTER*13 NUCA(NNUCS), MUCT(NBMAX), NUCBLK

DOUBLE PRECISION CIPF(NNUCS), CIT(NBMAX), GPF

c

CIPF $=\mathrm{mCi} / \mathrm{Cu}-\mathrm{ft}, \mathrm{CIT}$ is $\mathrm{Ci}, \mathrm{GPF}=\mathrm{g} / \mathrm{cu}-\mathrm{ft}$

DIMENSION IBIG(NNUCS), IFIS(NNUCS), TOTACT(NNUCS), ATNO(NNUCS),

$+$ HALF (NMUCS)

LOGICAL TRU, ALF, FIS(NNUCS), BIGACT

logical debug

DATA NUCBLK/"

$1 /$

C**** check file counts!

IFBASE + 1 is first output file,

IIBASE +1 is first nonfissile output file

This data statement is for 6 fissile nuclides $>2 \mathrm{Ci}$

DATA IFBASE/6/,11BASE/12/

C Anyone tho modifies this progran should then run it with

C debuga.true. before trying it with debuga. false.

C Setting debug=.true. limits the andysis to 20 shipments, and

C generotes lots of printout for those shipments.

data debug/. false.l

C

Statement function defining "big" activities

$\operatorname{BIGACT}(N)=$ TOTACT $(N)$.GT. 2

C CITOG * Activ(in mCi) * Halflife(in d) * Atomic number = Mass(in g)

CITOG $=(2.798 E-9) / 365.24$

OPEN (UNIT=6, FILE= 'midbo.prp')

OPEN (UNIT=4, FILE='nucinf. dat' ')

DO $20 \mathrm{~N}=1$, NMUCS

Get muclide name, half-life (d), properties and total activity

$\operatorname{READ}(4, *) \operatorname{MUCA}(N), \operatorname{HALF}(N), \operatorname{TRU}, A L F, F I S(N), \operatorname{TOTACT}(N)$

C Get atomic numbers

if (debug) write(6, '(''nuca(n)=1',a)') nuca(n)

READ(NUCA(N), ' (A6)') PART

if(debug) write $(6, '$ ' '' part $=11, a) '$ ') part

IF (PART.EQ. 'LES-TH') THEN

Arbitrary atomic number

ATHO(N) $=100$

ELSE IF (PART.EQ.'MAP 1) THEN

Treat as $\mathrm{CO}-60$

ATMOCN $=60$

ELSE IF (PART.EQ. 'MFP ") THEN

C Treat as half $\mathrm{Sr}-90$, half $\mathrm{Cs}-137$ $\operatorname{ATMO}(N)=113.5$

c

ELSE IF (PART.EQ. 'UN-1D-1) THEN Treat as Cs -137 


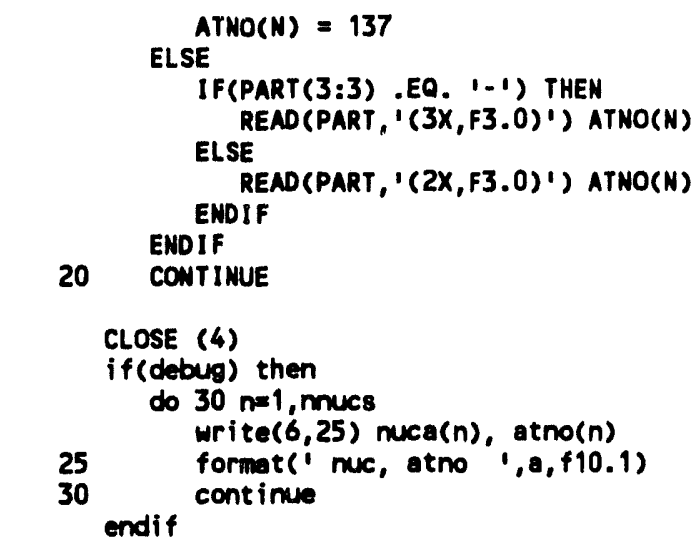

C Set up mapping to compressed array of fissile nuclides NBIGF $=0$

DO $40 \mathrm{~N}=1$, NNUCS

IF(FIS(N) .AND. BIGACT(N)) THEN

NBIGF = MBIGF +1

ELSE

IFIS $(N)=$ NBIGF

$\operatorname{IFIS(N)}=0$

ENDIF

if (debug) write(6, '(1' ifis('1,i2, '1)=1',i3)') n, ifis(n)

40 CONTINUE

IF (NBIGF.GT . NFMAX) THEN

WRITE $(6,45)$ NFMAX

45 FORMAT(' Error. Set mfmaX >1, 14)

STOP

ENDIF

C Set up mapping to compressed array of nuclides

NBIG $=0$

DO $50 \mathrm{~N}=1$, NNUCS

IF(BIGACT(N)) THEN

NBIG = NBIG +1

IBIG(N) = NBIG

ELSE

$I B I G(N)=0$

ENDIF

if(debug) write $(6,1(11$ ibig( $11, i 2,11)=11, i 3) 1) n$, ibig(n)

50 CONTINUE

IF (NBIG.GT.NBMAX) THEN

WRITE $(6,55)$ NBMAX

55 FORMAT(' Error. Set NBMAX >1, 14) STOP

ENDIF

DO 950 IRUN=1,3

C Do this in 3 runs, because Lahey Fortran with Dos only

C allows 20 files open at a time

IF(IRUN.EO.1) THEN

IRBASE $=6$

IRMANY $=13$

IRTOP = IRBASE + IRMANY

CHthardwired output file names

C****morder of nuclides must match nuclides $>2 \mathrm{Ci}$ in nucinf.dat

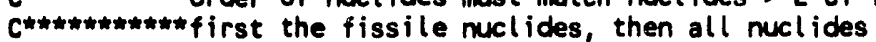

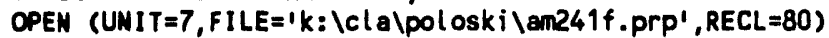

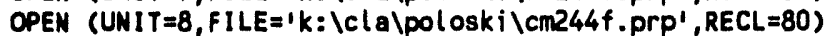

OPEN (UNIT $=9, F I L E=1 k$ : $\backslash \mathrm{cl}$ la $\backslash$ pol oski $\backslash$ pu238f. prP',$R E C L=80$ )

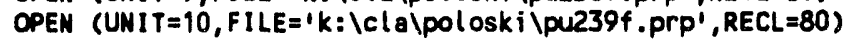

OPEN (UNIT $=11, F I L E=1 k$ : $\backslash c$ la lpoloski 1 PU241f, PrP', RECL $=80$ )

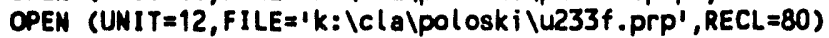

OPEN (UNIT $=13, F I L E=1 k$ : (cla\poloski $\backslash a m 241$. prP', RECL $=80$ ) 
OPEN (UNI $T=14, F I L E=1 k:|c l a| p o l o s k i \backslash b a 137 m$. prp', RECL $=80$ )

OPEN (UNI $T=15, F I L E=1 k: \backslash c l a \backslash p o l o s k i \backslash b i 212 . p r p ', R E C L=80$ )

OPEN (UNI $T=16, F I L E=1 k:|c l a| p o l o s k i \mid c e 144, p r p ', R E C L=80$ )

OPEN (UNIT $=17$, FILE $=1 \mathrm{k}: \mid \mathrm{cl}$ (a)poloski $\backslash \mathrm{cm} 244 . \mathrm{prp} 1, \mathrm{RECL}=80$ )

OPEN (UNIT $=18, F I L E=1 k$ : $\backslash c$ la $\backslash$ pol óski $\backslash C 060$. PrP',$R E C L=80$ )

OPEN (UNI T=19, FILE=' $k:|c l a| p o l o s k i \mid c s 134, p r p ', R E C L=80$ )

DO $60 \mathrm{~N}=1$, NNUCS

IF(IFIS(N) .GT. O) THEN

IUN = IFBASE + IFIS(N)

IF(IUN.GT.IRBASE .AND. IUN.LE.IRTOP) THEN

WRITE(IUN, ' $(1 X, A, 1$ ' fissile'' ')' $\operatorname{MUCA}(N)$

if(debug) write( 6 ,

$+$

60$$
\text { ENDIF }
$$

if(debug) write(6, '('' above loop 65'1)')

DO $65 \mathrm{~N}=1$, NNUCS

IF(IBIG(N) . GT. O) THEN

IUN = IIBASE + IBIG(N)

IF(IUN.GT.IRBASE .AND. IUN.LE.IRTOP) THEN

WRITE(IUN, ' $(1 X, A) ')$ MUCA(N)

if(debug) write( 6 ,

'('' ibig('', i2, '') = '', i3,9x,a)' )

$+$ $n$, ibig(n), nuca(n)

65 CONTINUE

ELSE IF(IRUN.EQ.2) THEN

IRSASE $=$ IRTOP

IRMANY $=13$

IRTOP = IRBASE + IRMANY

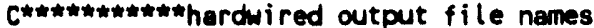

OPEN (UNIT=20, FILE =' $k$ : $\backslash c l a \backslash p o l o s k i \backslash c 8137 . p r p ', R E C L=80$ )

OPEN (UNIT $=21, F I L E=' k: \mid c l$ a $\backslash$ pol oski $\backslash k r 85 . p r p ', R E C L=80$ )

OPEN (UNIT $=22, F I L E=1 k$ : $\backslash c$ la $\backslash p o l o s k i \backslash m f p . p r P ', R E C L=80$ )

OPEN (UNIT $=23$, FILE $=1 k:|c l a| p o l o s k i \backslash n i 63 . p r p ', R E C L=80$ )

OPEN (UNIT $=24$, FILE =' $k$ : $\backslash c l a \backslash p o l o s k i \backslash p b 212$.prp', RECL $=80$ )

OPEN (UNI T $=25, F I L E=1 k:|c l a| p o l$ osk i $\backslash p m 147 . p r P ', R E C L=80$ )

OPEN (UNIT $=26, F I L E=1 k:|c l a| p o l o s k i \backslash p 0212$. prP', RECL $=80$ )

OPEN (UNIT $=27, F I L E=' k:|c l a| p o l o s k i \mid p 0216 . p r p ', R E C L=80$ )

OPEN (UNIT $=28, F I L E=' k:|c l a| p o l o s k i \mid p r 144 . p r P ', \quad R E C L=80$ )

OPEN (UNIT $=29, F I L E=' k$ : $|c| a \mid p o l o s k i \backslash p U 238 . p r p ', R E C L=80$ )

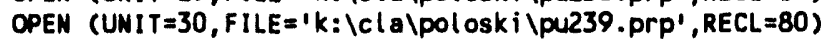

OPEN (UNI $T=31, F I L E=1 k:|c l a| p o l o s k i \backslash p u 240 . p r P ', R E C L=80$ )

OPEN (UNIT $=32, F I L E=' k:|c l a| p o l o s k i \backslash$ pU241, prP',$R E C L=80$ )

DO $70 N=1$, NNUCS

IF(IFIS(N) . GT, O) THEN

IUN = IFBASE + IFIS(N)

IF(IUN.GT.IRBASE .AND. IUN.LE.IRTOP) THEN

MRITE (IUN, ' $(1 X, A)$ ') MUCA $(N)$

if (debug) write( 6 ,

$+$

'(' ifis $(1, i 2,1 ')=11, i 3,9 x, a) ')$

ENDIF

ENDIF

$n$, ifis(n), nuca(n)

70 CONTINUE

DO $75 \quad N=1$, NNUCS

IF(IBIG(N) .GT. O) THEN

$I U N=I I B A S E+I B I G(N)$

IF (IUN.GT. IRBASE . AND. IUN.LE.IRTOP) THEN

WRI TE (IUN, ' $\left.(1 X, A)^{\prime}\right)$ NUCA(N)

if (debug) write( 6 ,

$+\quad$ '(' ${ }^{\prime \prime}$ ibig(' $\left.\left.1, i 2,{ }^{\prime \prime}\right)=11, i 3,9 x, a\right) '$ )

$+n$, ibig(n), nuca(n) 


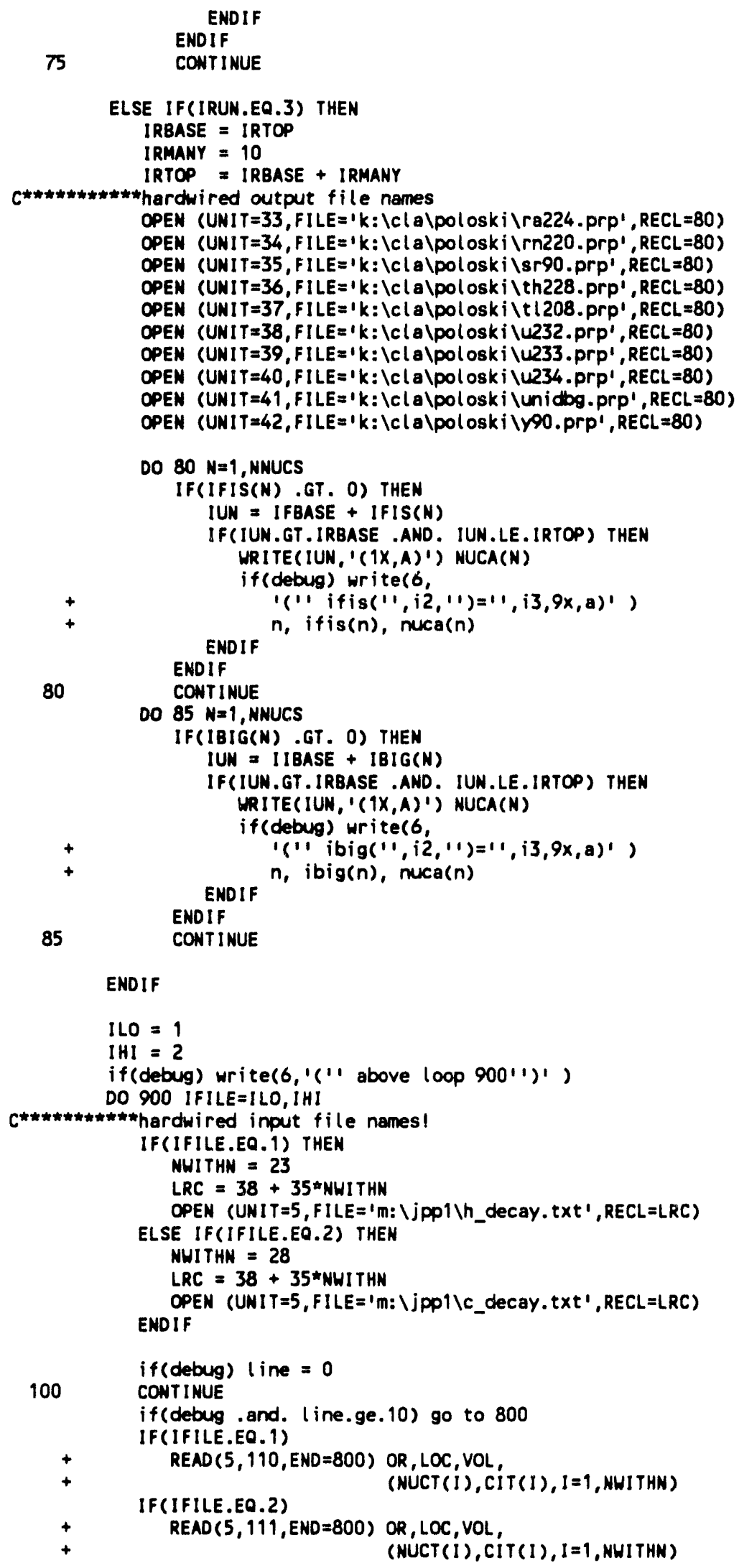




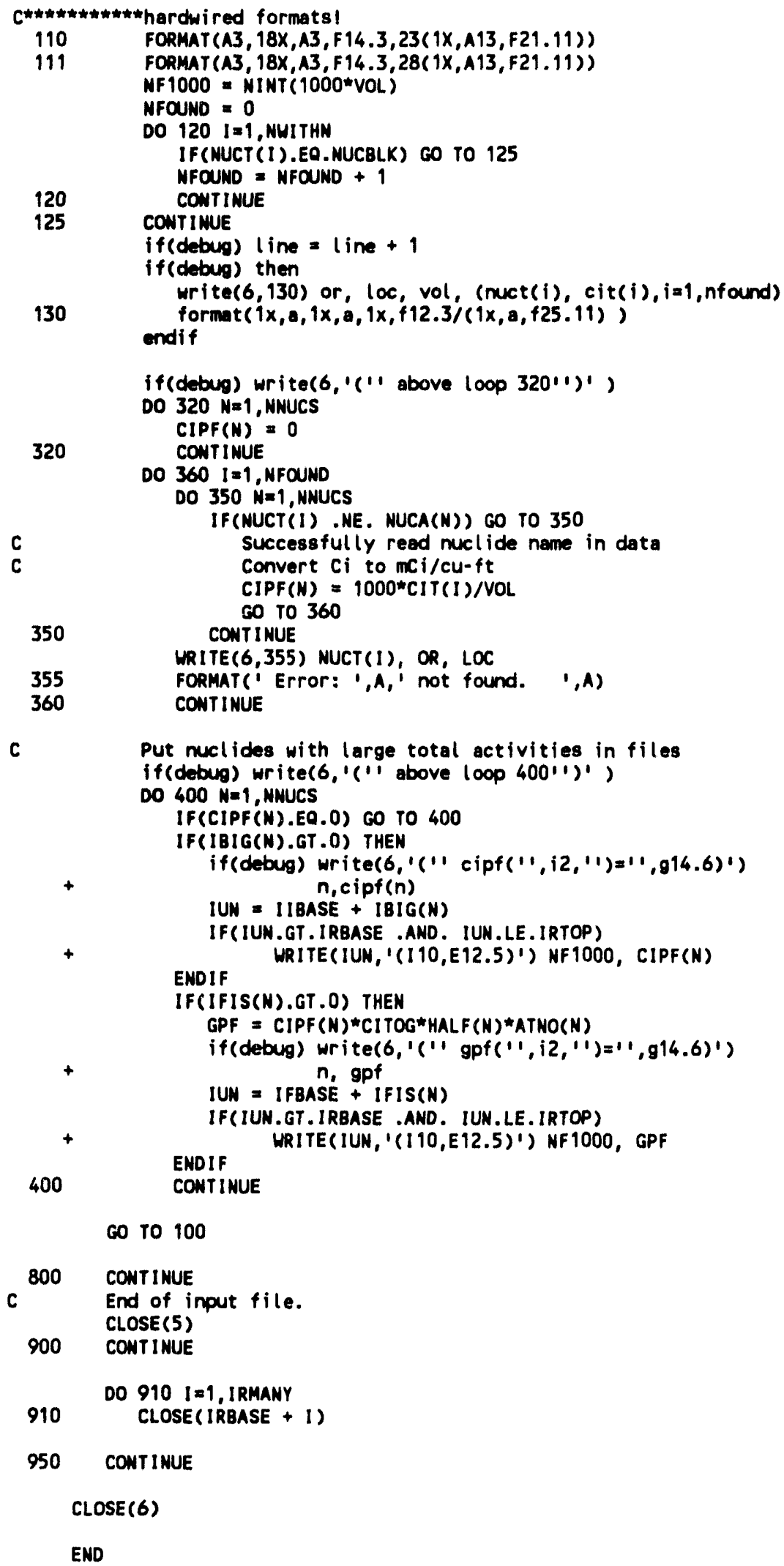


PROGRAM PREPD

Reads text-file from dBASE massage of RuMIS download, and normalizes by cubic foot

Calculates dose from intalation, besed on dose conversion factors

WRITTEN BY C. ATWOOD, Feb-Apr 1993

IMPLICIT REAL(A-H, O-Z), INTEGER(I-N)

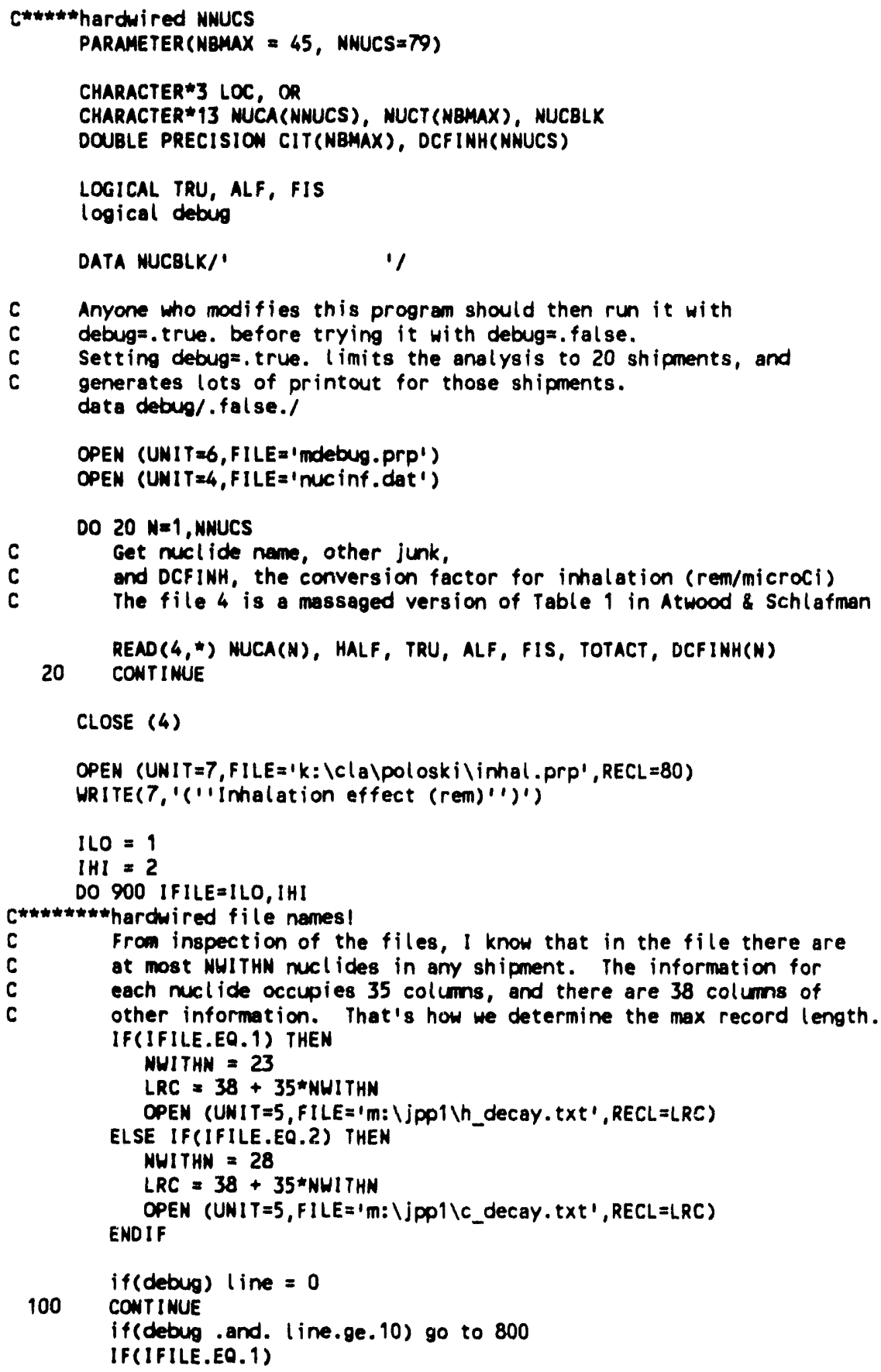




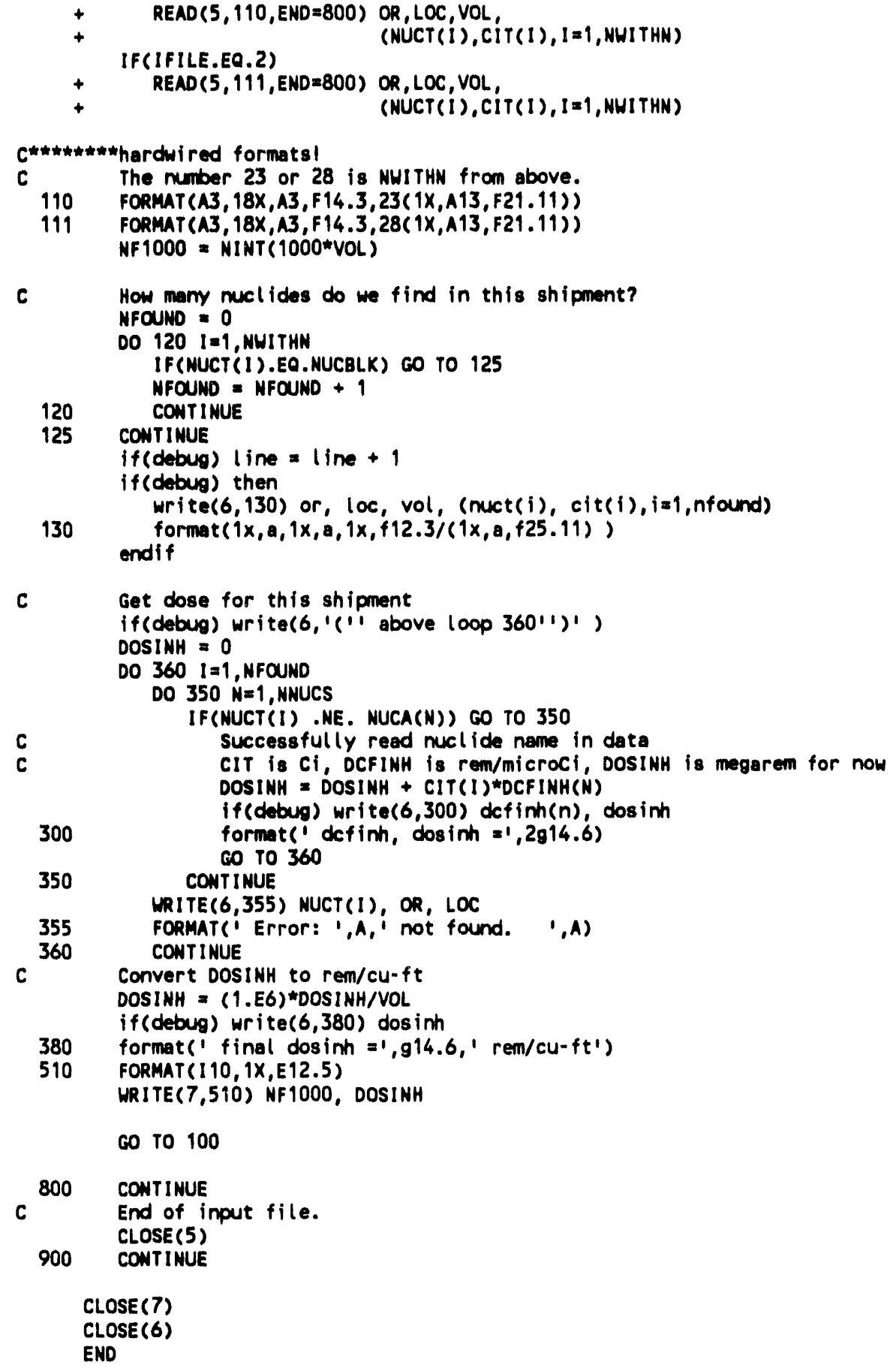

PROGRAM TALLY

Reads text-file from dBASE massage of RWMIS download Counts shipments by originator, by location, and by nuclide 
IMPLICIT DOUBLE PRECISION(A-H,O-Z), INTEGER(I-N)

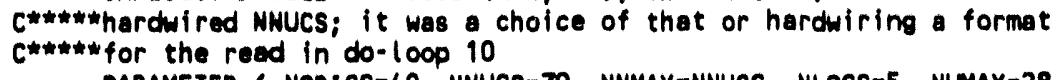

PARAMETER ( NORIGS=40, NNUCS $=79$, NMMAX $=$ NNUCS, NLOCS=5, NMMAX=28)

CHARACTER $\$ 3$ OR, ORA(NORIGS), LOC, LOCA(NLOCS)

CHARACTER*13 NUC(NMMAX), NUCT (NLWAX), NUCBLK

CHARACTER 13 DECOMP(5)

DIMENSION KNTO(NORIGS,3), KNTN(NMMAX), KNTL(NLOCS,3), KNTOTS(5), $+$ KNTFIS(3)

OIMENSION VOLO(NORIGS,3), VOLN(NMMAX), VOLL(NLOCS,3), VOLTOT(5),

$+$ VOLFIS(3)

DIMENSION HALF( WHMAX)

DOUBLE PRECISION CIN(NMMAX), CIO(NORIGS,3), CIL(NLOCS,3),

$+\quad$ CIfIS(3), Citots(5),

+ CIT(nLmaX), TOTAC(NMmaX), DRCl, DROUND

LOGICAL TRU(NMMAX), ALF(NMMAX), FIS(NMMAX)

LOGICAL COTTRU, GOTNOT, GOTALF, GOTBEG, GOTFIS, GOTTF, GOTNF

logical debug

data debug/.false.l

DATA KLOC/O/, KOR/O/

DATA NUCBLK /1
DATA DECOAP /1 Total', TRU', Non-TRU', Alpha', Beta/gamma'/

OPEN (UNIT $=7$, FILE=' decaym.tal' ')

OPEN RUNIT $=4, F I L E=$ 'nucinf.dat')

DO $10 \mathrm{~N}=1$, NMUCS

C

Get nuclide neme, half-life (d), properties and total activity

$+$

$\operatorname{READ}(4, *) \operatorname{NUC}(H), \operatorname{HALF}(N), \operatorname{TRU}(N), \operatorname{ALF}(H), \operatorname{FiS}(N)$,

10

CONTINUE TOTAC (N)

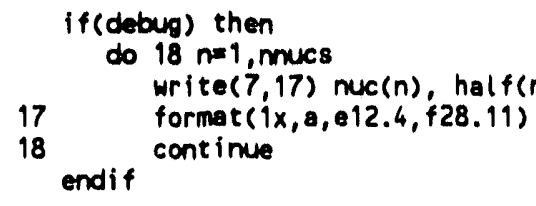

DO $30 \mathrm{~N}=1$, NAUCS $\operatorname{KNTN(N)}=0$ $\operatorname{VOLN}(N)=0$ $\operatorname{CIH}(N)=0$

30 CONTINUE

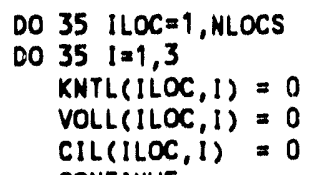




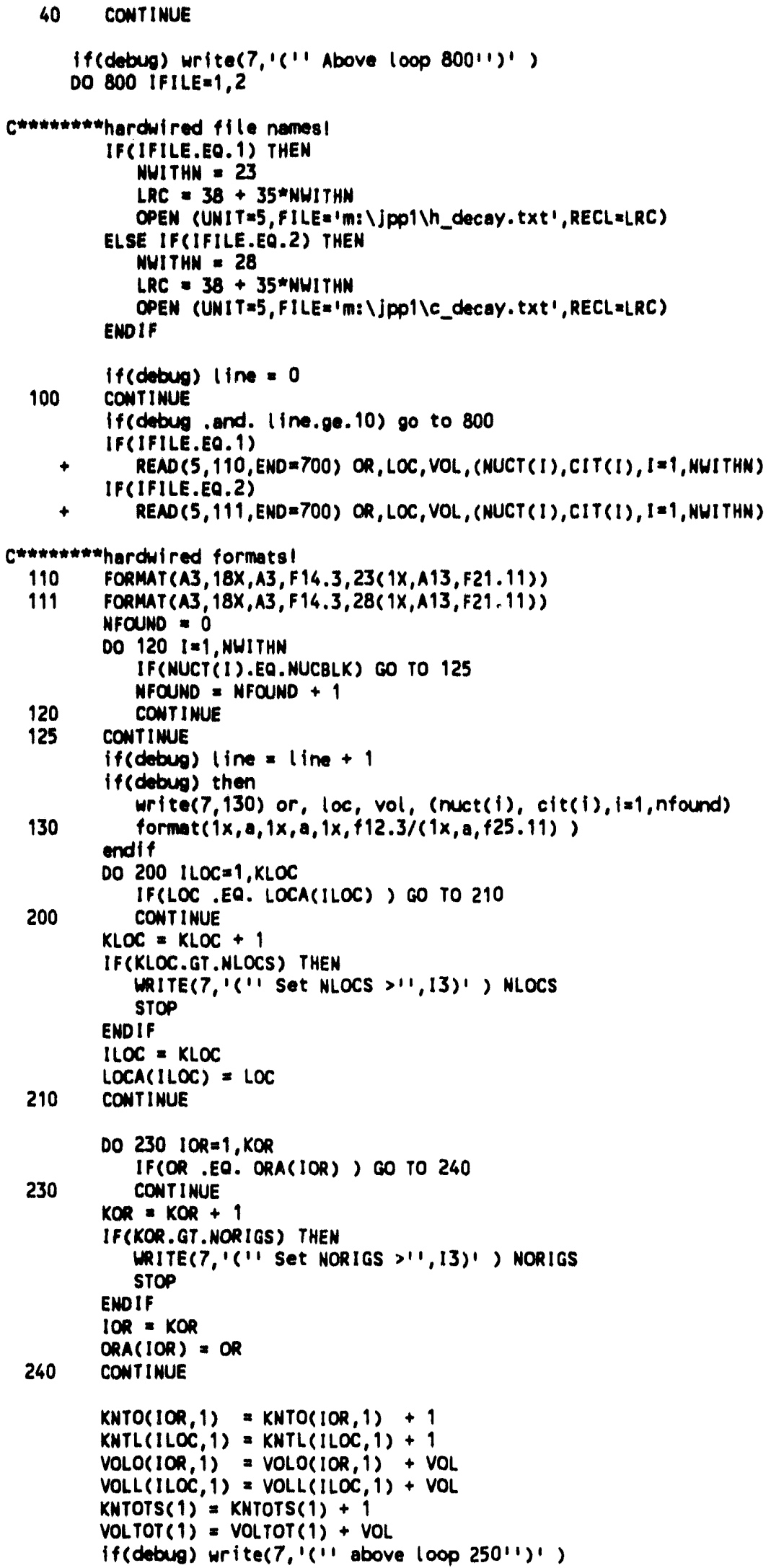




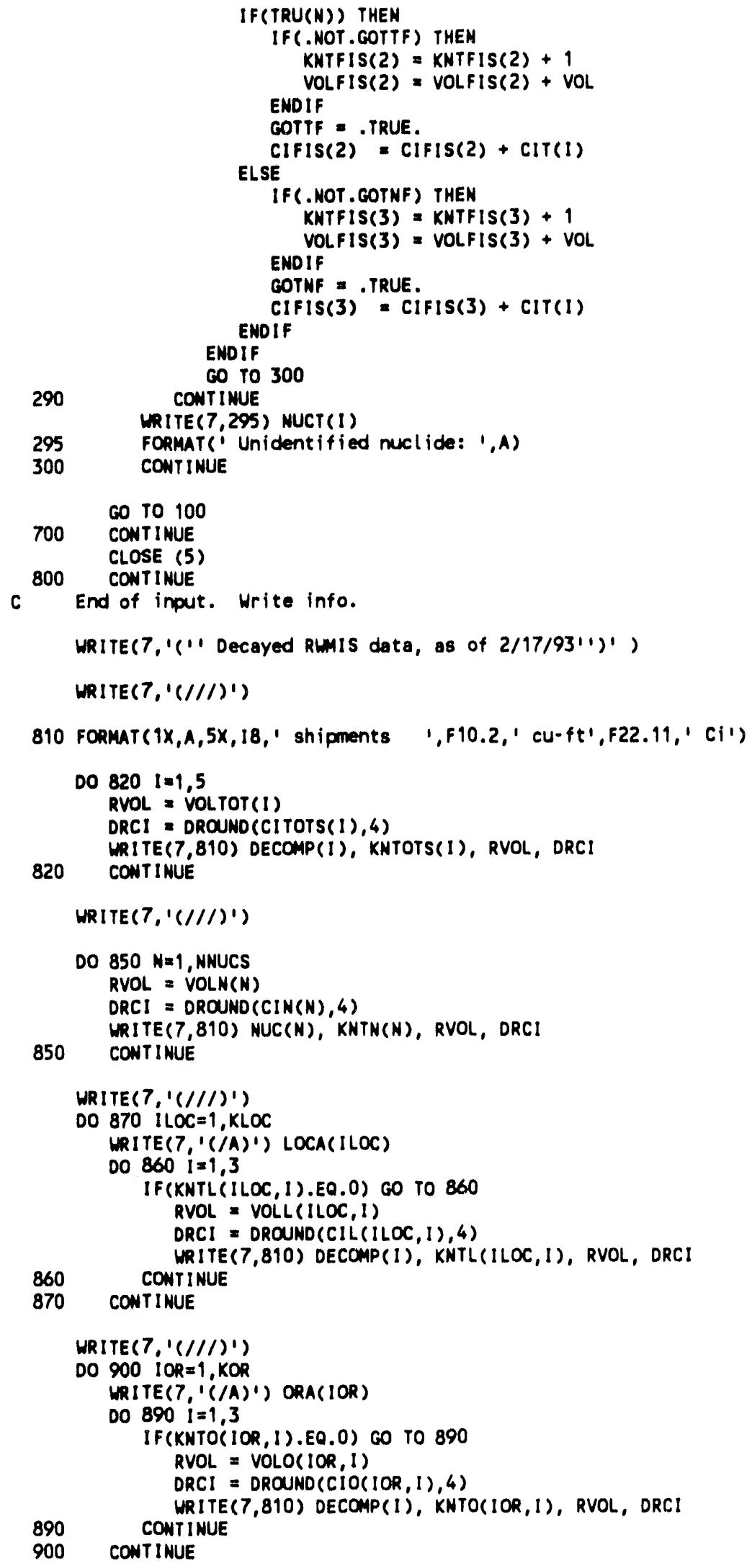


$\operatorname{MRITE}(7,1(/ / /)$ ')

WRITE(7,' ('1 Fissile nuclides'1)')

DO $910 \quad i=1,3$

IF(KNTFIS(I).EO.0) 60 TO 910

RVOL = VOLFIS(I)

DRCI = DROUND(CIFIS(1),4)

910 CONTIMUE

MITE(7,810) DECOAP(1), KNTFIS(1), RVOL, DRCI

985 CONTINUE

CLOSE (5)

990 CONTINUE

CLOSE(7)

END

DOUBLE PRECISION FUNCTION DROUNO $(X, N)$

C Rounds double precision numer to $N$ significant digits, for $F$ printing

C Must have $0<N<10$.

IMPLICIT DOUBLE PRECISION(A-H,O-Z), INTEGER(I-W)

CHARACTER*7 FMT

CHARACTER $\$ 16$ STRING

IF(N.LE.O) THEN

WRITE(6, '(1" Illegal input to OROUNO, $\left.N=11,15)^{\prime}\right) \quad N$

DROUND $=x$

ELSE IF(N.LE.9) THEN

WRITE(FMT , 20) N

20 FORMAT( '(E16.1,11,1)'

WRITE(STRING, FMT) $X$

READ (STRING ऋ) DROUND

ELSE

DROUND $=x$

ENDIF

RETURN

END

PROGRAM PMZERO

Reads output of TALLY, finds fraction of $\mathrm{cu}-\mathrm{ft}$ (out of total cu-ft)

for each item whose empirical distribution is found

WRITTEN BY C. ATHODD, March 1993

IMPLICIT REAL (A-H,O-Z), INTEGER(I-N)

CHARACTER 22 NAME

CHARACTER 1 MARKER, BLANK

DOUBLE PRECISION CUFT, DENOM, TOTACT, FRAC

LOGICAL FIRST

logical debus

data debug/.false./

DATA BLANK/" '/

OPEN (UNIT=5, FILE=' decaym.tal' ')

OPEN (UNIT=6, FILE=' 'pnzero.txt')

FIRST $\approx$.TRUE,

50 FORMAT $(A, F 22.11)$

100 CONTINUE

READ $(5,110, E N D=700)$ NAME, MARKER, CUFT, TOTACT

110 FORMAT $(A, 7 X, A, 7 X, F 13.2,6 X, F 22.11)$ 


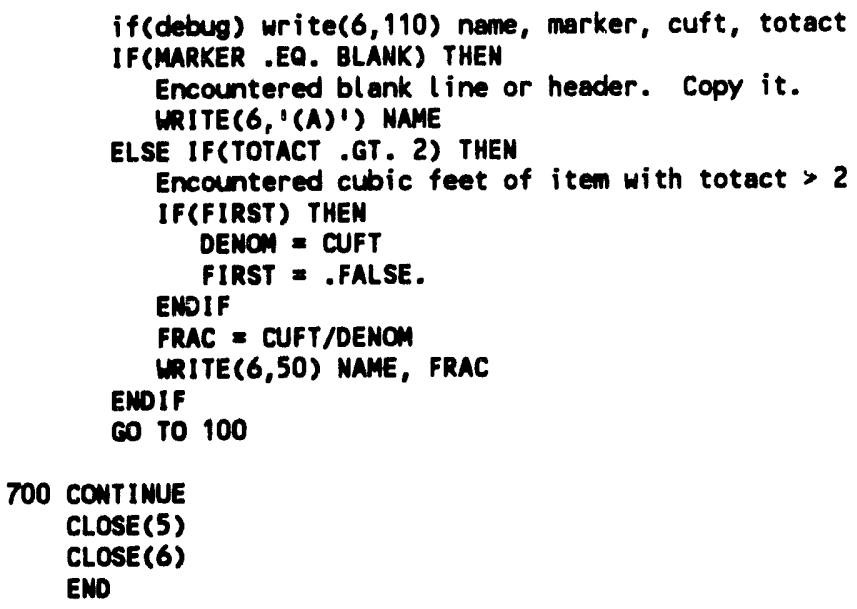

\section{SAS Programs to Obtain Empirical Distributions}

Four SAS (1988) programs, differing only in small detail, were written for obtaining the quantiles of the empirical distribution. They are LOCOR, for the files produced by PREP giving data by storage location and by waste originator, FISSIL for the files produced by PREP and PREPI for the fissionable nuclides, ALLELSE for all other nuclide activities produced by PREPI, and DOSE for the committed effective dose equivalent. The SAS programs are reproduced below. When the input file was short, the program could be run under DOS on an IBM PS/2 Model 70 386. When the input file was long, it was necessary to run the program in an UItrix environment on a DECstation 5500. Therefore, the files read by the programs were not precisely as shown here; files in directory $k: \backslash c l a \backslash p o l o s k i$ were located on the $P C$ server, while files with no directory shown were located on the DECstation, and some programs were run both places to pick up the appropriate files.

Only a brief effort was deemed necessary for verifying and validating the SAS programs, because SAS is a widely used commercial package and the programs used here were short and simple. Nonetheless, the empirical distributions and maximum values found were compared with the data for some of the nuclides 1 isted in Table 5, and the SAS results were consistent with the data. The SAS programs are listed here. 
PROC PRINT

RUN

XUEND empirm

Xempirm(fis,All Fissionable)

Xempirm(truf, TRU Fissionable)

Xempirm(notf, Non-TRU F issionable)

Xempi rm( em241f,Am-241)

Xempi rm(cm244f, $\mathrm{Cm}-244)$

Xempi rm(pu238f, Pu-238)

Xempi rm(pu241f, Pu-241)

Xempirm(u233f,U-233)

/* Progrem ALLELSE */

OPTIONS PAGESIZE $=80$ LINESIZE $=120$

XMACRO empirm (ifile, ititl)

TITLE "Empirical distr for Bititl, mCi per cu-ft"

DATA dat

IMFILE "k: lclalpoloskil\&ifile..prp" FIRSTOBS=2

INPUT nf1000 activ

1* nf1000 = $1000 \times$ cu-ft in shipment

activ is $\mathrm{mCi} / \mathrm{cu}-\mathrm{ft}$

-

PROC UNIVARIATE

var activ

freq nf 1000

OUTPUT petlpts=10 $203040506070 \quad 80909899.599 .9$ petlpresp

RUN

PROC PRINT

RUN

MUEND EIPITI

Xempi rm(all, All)

Xempi rm(tru, TRU)

Xempi rm(not, Non- TRU)

Xempi rm(alph, Alpha)

Xempi rm(bega,Beta/Gemma)

Xempi rm(AM241,Am-241)

Xempi rm(BA137M,Ba-137M)

Xempi rm(BI212, Bi -212)

Xempi rm(CE 144, Ce-144)

Xempi rm(CH244, $\mathrm{Cm}-244$ )

Xempi im(COsO, Co-60)

Xempirm(Cs134, Cs-134)

Xempi rm(CS137, C8-137)

Xempi rm(KR85, Kr-85)

Xenpi rm(MFP, MFP)

Xenpi rm(N163, Mi-63)

Xempi rm(PA234M, Pa-234M)

Xempi $r m(P B 212, P b-212)$

Xempi rm(PM147, Pm-147)

Xempi rm(P0212,Po-212)

Xempi rm(PO216,Po-216)

Xempi rm(PR144, Pr-144)

Xempirm(PU238, Pu-238)

Xempi rm(PU239,Pu-239)

Xempi rm(PU240,Pu-240)

Xempi rm(PU241, Pu-241)

Xenpi rm(RA224,Ra-224)

Xempirm(RN220,Rn-220) 
SAS Institute, Inc., 1988, SAS Language Guide for Personal Computers, Release 6.03 Edition, Cary, NC: SAS Institute Inc.

Schl afman, M. J., 1992, letter to C. M. Hiaring, "Pit 10, Pit 13, and Trench 32 TRU RWMIS Data", MJS-20-1992, November 24,1992.

Stephenson, R., 1958, Introduction to Nuclear Engineering, New York: McGraw-Hill, pp. 28-31.

Walker, F. William, Dudley G. Miller, and Frank Feiner, 1984, Chart of the Nuclides, Thirteenth Edition, San Jose: General Electric Company, Nuclear Energy Operations. 
$\mid$

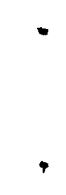




\section{APPENDIX B: ANALYSIS METHODS TRIED}

Several methods were attempted to analyze data for Pu-239 from Rocky flats. The results are described here. The more ambitious methods turned out to be unworkable for this restricted data set, and therefore were not attempted for the full RWMIS data. The method called Approach (4) below is the method used in the body of this report, except that the body of the report works with cubic feet instead of gallons.

Each shipment corresponds to one or more records in RWMIS. A record corresponds to a specified number of containers of a given size, for example six 55-gal containers. If the shipment contained various sizes of containers, there is at least one record for each container size. If the containers, even containers of the same size, contain different waste types, or if they are now stored at different locations, or if they differ in other ways deemed noteworthy, there can be many records for the containers, up to one record per container. However, the activity given for the shipment is not broken down by container, or even by record; instead, the only activity reported is the total activity for the shipment. The fundamental problem in analyzing the data is apportioning the total activity for a shipment among the containers.

\section{First Techntaues Tried}

At first, consider shipments that only have one kind of container, such as only 55-gal drums, and estimate the moments of the distribution of the activity in a random container. Three approaches were tried:

(1) Assume that all the containers within a shipment have exactly the same activity. This assumption gives an estimated activity for each container. The resulting distribution of activities is the empirical distribution.

(2) Assume that the containers of one type are generated by a process, which produces variability both between shipments and between containers within a shipment. Manipulation of formulas (not shown here) then yields 
unbiased estimators of the mean and the variance of the process.

(3) Some consecutive shipments have exactly the same inventory per container. Call these groups of shipments "super-shipments," and treat them the way shipments are treated in Approach (2). In the Current Data, there are 17,000 shipments but only 10,000 super-shipments; many individual containers are listed as separate shipments, and apparently a single estimated activity was often deliberately used for many such containers officially listed as in different shipments.

The three approaches yield the same estimate of the mean activity, and different variances. Approach (1) gives the smallest estimated variance, because it sets the variability within shipments to zero, and Approach (3) gives the largest. The magnitude of the difference is suggested by the following examples: For containers from Rocky Flats in the Current Data, we have

55-gal Drums

$\begin{array}{ccc}\text { Approach } & \frac{\operatorname{Rel} \cdot \text { s.d. }}{2.40} & \frac{\text { Basis }}{16568 \text { containers }} \\ \text { (1) } & 2.61 & 13891 \text { shipments } \\ \text { (2) } & 3.76 & 6743 \text { super-shipments } \\ \text { (3) } & 3.76 & \end{array}$

\begin{tabular}{ccc} 
& \multicolumn{2}{c}{$112-\mathrm{cu}-\mathrm{ft}$ Containers } \\
\cline { 2 - 2 } & $\frac{\text { Rel. S.d. }}{1.21}$ & $\frac{\text { Basis }}{3149 \text { containers }}$ \\
(1) & 1.35 & 2529 shipments \\
(3) & 1.36 & 2497 super-shipments
\end{tabular}

The next natural step is to try to fit a parametric distribution. The mean and variance, estimated above, can be used to estimate the parameters; this works very neatly when fitting a gamma distribution, because the parameters of a gamma distribution are simple functions of the mean and variance. Alternatively, two quantiles of the empirical distribution can be used to fit a two-parameter distribution; this works very neatly when fitting a lognormal distribution or a truncated lognormal.

\section{Problems with the first techniques}

Parametric fits do not necessarily work well. Neither a gamma nor a 
lognormal distribution gives a good fit to the Rocky Flats (RFO) Current Data for 55-gal drums or for 112-cu-ft containers. (For each data set the theoretical tails are too long, or equivalentiy, the observed tails are too short.) A truncated lognormal fits the 55-gal drum data, but nothing has been found that fits the 112-cu-ft container data. Therefore, the empirical distribution seems to be the only option for a general method. Unfortunately, if the model of Approach (2) or (3) is correct, the empirical distribution understates the variance somewhat.

The approaches (1)-(3) are designed for shipments that have only one kind of container. However, some shipments contain several kinds of containers (in the Historical Data, not in the Current Data). This is not a trivial issue. For example, in the Historical Data, RFO generated ninety-four 30-gal barrels. Not one of these barrels came in a shipment of only 30-gal barrels; instead, every one came in a shipment with many 55-gal barrels. Typically a shipment contained one 30-gal barrel and thirty-five 55-gal barrels, or else two 30-gal barrels and seventy 55-gal barrels. This makes it impossible to infer the distribution of activities of RFO 30 -gal barrels without making some assumption about the relation of the contents of the two kinds of containers. Typically, the two kinds of containers in one shipment contained qualitatively different waste.

In addition to the main generators and the main container types, there are numerous other generators and container types. For example, there is one container from CFA, one from CPP, etc.; similarly, there is one 378-cu-ft container, one 2-gal container, etc. This is not a serious methodological problem, but it is a nuisance if we try to obtain a separate distribution for each container type or each generator.

\section{Next Techniques Tried, and Ultimately Used}

(4) Assume that each shipment is homogeneous, and obtain the empirical distribution of activity per gallon. This allows us to deal with shipments containing a variety of container types. One could work with the gallon or the cubic foot as the basic unit; the gallon is smaller, and 
therefore better avoids the problem of shipments with fractional units.

This approach yields the correct overall mean per gallon. It underestimates the variance because it uses the empirical distribution, based on the assumption that each shipment is perfectly homogeneous. To simulate a container using this method, draw a random activity per gallon from the distribution, then multiply that activity by the number of gallons in the container.

To increase the estimated variance, consider a fifth approach:

(5) Assign different activities to otherwise identical containers within a shipment, so that the total activity is the activity reported by RWMIS, and the individual activities follow a uniform distribution. (The uniform distribution is chosen for simplicity. To make it as wide as possible, given the mean activity, let the lower limit be zero, and therefore let the upper limit be twice the mean.)

When Approach (5) was followed for every shipment with the Pu-239 data, the resulting file had one line per container, and was too large to be manageable on a PS/2 Model 70 with a PS/2 Model 80 Server. If instead it was done only for the shipments with relatively large activity (shipments with multiple containers and mean activity/gallon $>2 \mathrm{mCi} / \mathrm{gal}$ ), the data file was tripled in length, but could still be analyzed by SAS on a DEC workstation. The resulting distribution differed only moderately from the empirical distribution, as shown below.

Overall distribution of $\mathrm{Pu}-239, \mathrm{mCi} / \mathrm{gal}$

\begin{tabular}{|c|c|c|}
\hline \multicolumn{2}{|c|}{ Value of $\mathrm{Pu}-239$ (mCi/gal) } & Cumulative \\
\hline Approach (4) & Approach (5) & Probability \\
\hline & 0 & 0.0 \\
\hline $\begin{array}{l}0.000082 \\
0.00886\end{array}$ & $\begin{array}{l}0.000083 \\
0.00886\end{array}$ & $\begin{array}{l}0.10 \\
0.20\end{array}$ \\
\hline 0.0265 & 0.0265 & 0.30 \\
\hline $\begin{array}{l}0.206 \\
0.619\end{array}$ & $\begin{array}{l}0.206 \\
0.596\end{array}$ & $\begin{array}{l}0.40 \\
0.50\end{array}$ \\
\hline 0.930 & 0.876 & 0.60 \\
\hline 1.34 & 1.19 & 0.70 \\
\hline $\begin{array}{l}2.41 \\
5.16\end{array}$ & $\begin{array}{l}1.88 \\
5.0 ?\end{array}$ & 0.80 \\
\hline 15. & 17.9 & 0.98 \\
\hline
\end{tabular}


The empirical distribution, from Approach (4), has mean $2.098 \mathrm{mCi} / \mathrm{gal}$ and standard deviation $6.257 \mathrm{mCi} / \mathrm{gal}$. The distribution modified by Approach (5) has the same mean, and standard deviation $6.417 \mathrm{mCi} / \mathrm{gal}$. The modification does not differ enough from the empirical distribution to be worth the effort.

The theoretically correct version of Approach (5) would not use the uniform distribution, but instead would use the conditional distribution of the container activities, conditional on the sum of the activities, and estimated by using the empirical distribution for the unconditional distribution. Though theoretically correct, this method would be extremely difficult to implement. Therefore the uniform distribution was used instead of the conditional distribution. The uniform distribution is symmetrical, unlike the presumably skewed conditional distribution, and has a smaller relative standard deviation. Use of the uniform distribution results in only modest changes from the empirical distribution; use of the theoretically correct conditional distribution would probably result in greater changes, but it is not known how great. 

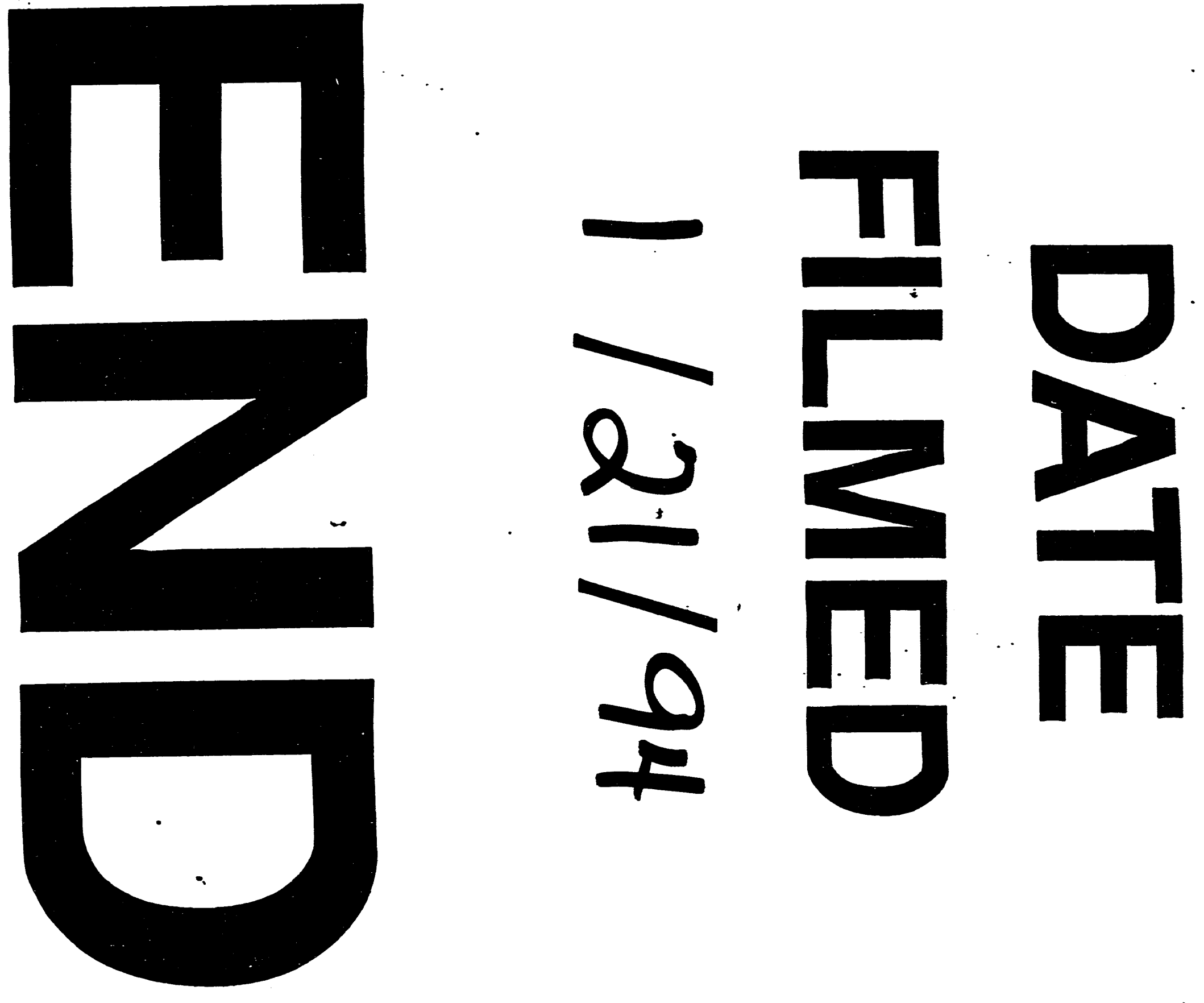
